\title{
A VARIATIONAL FORMULATION FOR COMPUTING SHAPE DERIVATIVES OF GEOMETRIC CONSTRAINTS ALONG RAYS
}

\author{
Florian Feppon ${ }^{2}$, Grégoire Allaire ${ }^{1}$ And Charles Dapogny ${ }^{3, *}$
}

\begin{abstract}
In the formulation of shape optimization problems, multiple geometric constraint functionals involve the signed distance function to the optimized shape $\Omega$. The numerical evaluation of their shape derivatives requires to integrate some quantities along the normal rays to $\Omega$, a challenging operation to implement, which is usually achieved thanks to the method of characteristics. The goal of the present paper is to propose an alternative, variational approach for this purpose. Our method amounts, in full generality, to compute integral quantities along the characteristic curves of a given velocity field without requiring the explicit knowledge of these curves on the spatial discretization; it rather relies on a variational problem which can be solved conveniently by the finite element method. The well-posedness of this problem is established thanks to a detailed analysis of weighted graph spaces of the advection operator $\beta \cdot \nabla$ associated to a $\mathcal{C}^{1}$ velocity field $\beta$. One novelty of our approach is the ability to handle velocity fields with possibly unbounded divergence: we do not assume $\operatorname{div}(\beta) \in L^{\infty}$. Our working assumptions are fulfilled in the context of shape optimization of $\mathcal{C}^{2}$ domains $\Omega$, where the velocity field $\beta=\nabla d_{\Omega}$ is an extension of the unit outward normal vector to the optimized shape. The efficiency of our variational method with respect to the direct integration of numerical quantities along rays is evaluated on several numerical examples. Classical albeit important implementation issues such as the calculation of a shape's curvature and the detection of its skeleton are discussed. Finally, we demonstrate the convenience and potential of our method when it comes to enforcing maximum and minimum thickness constraints in structural shape optimization.
\end{abstract}

Mathematics Subject Classification. 65K10, 49Q10, 36A15.

Received September 24, 2018. Accepted August 24, 2019.

\section{INTRODUCTION}

The recent achievements of shape and topology optimization techniques in predicting very efficient designs beyond the reach of the intuition of engineers have raised a tremendous enthusiasm in industry. One key issue about the practical use of these techniques is that they often lead to shapes which are too complicated to be assembled by traditional milling or casting processes, to the point that one of the greatest challenges faced

Keywords and phrases. Advection operator, shape and topology optimization, level set method, signed distance function, thickness constraints.

1 CMAP, CNRS, Ecole Polytechnique, Institut Polytechnique de Paris, 91128 Palaiseau, France.

2 Safran Tech, Magny-les-Hameaux, France.

3 Univ. Grenoble Alpes, CNRS, Grenoble INP (Institute of Engineering Univ. Grenoble Alpes), LJK, 38000 Grenoble, France.

*Corresponding author: charles.Dapogny@univ-grenoble-alpes.fr 
by shape and topology optimization is about the understanding and the implementation of manufacturing constraints. Such constraints generally bring into play the geometry of shapes; for instance, minimum thickness constraints, maximum thickness constraints, minimum distance between members, casting constraints have been considered; see $[16,38,50,62]$ or the recent survey [46].

Among the variety of methods proposed in the literature to enforce these constraints, a body of work has recently been devoted to formulate them by means of integral functionals involving the signed distance function $d_{\Omega}$ to the optimized domain $\Omega$, that is, the distance function to $\partial \Omega$ with a negative (resp. positive) sign inside (resp. outside) $\Omega$; see Section 3.1 below, and $[6,7,22,23,50]$ for the formulation of geometric constraints based on this concept. More precisely, the shape $\Omega$ is sought among all possible subsets of a fixed "hold-all" domain $D \subset \mathbb{R}^{n}$ ( $n=2,3$ in applications) as the solution of a constrained minimization problem of the form:

$$
\min _{\Omega \subset D} J(\Omega) \text {, s.t. } P(\Omega) \leq 0, \text { where } P(\Omega):=\int_{D} j\left(d_{\Omega}(x)\right) \mathrm{d} x,
$$

where $J(\Omega)$ is a measure of the performance of $\Omega$ (e.g. its elastic compliance), $j: \mathbb{R} \rightarrow \mathbb{R}$ is a given, smooth function, and $P(\Omega)$ is a geometric constraint. The above framework is quite appealing insofar as the signed distance function $d_{\Omega}$ naturally lends itself to clear mathematical formulations of maximum, minimum thickness constraint functionals (and the other aforementioned geometric criteria), thus making (1.1) amenable to an efficient numerical treatment by means of standard (e.g. steepest-descent) optimization algorithms. However, several technical stages in its numerical implementation pose difficulties; the leading motivation of the present article is to introduce a new variational method that make these substantially simpler to implement.

Let us provide a little more details about the main numerical issues raised by the implementation of (1.1) while staying at an explanatory level; see Sections 3.1 and 4 below for full details. The use of traditional optimization methods for the program (1.1) relies (in particular) on the knowledge of the shape derivative of the considered constraint functional $\Omega \mapsto P(\Omega)$. Throughout this article, this notion is understood in the framework of the Hadamard method: the shape derivative corresponds to the Fréchet derivative of $\theta \mapsto P\left(\Omega_{\theta}\right)$ at $\theta=0$, where local variations of $\Omega$ are considered under the form $\Omega_{\theta}=(\operatorname{Id}+\theta)(\Omega)$, using vector fields $\theta \in W^{1, \infty}\left(D, \mathbb{R}^{n}\right)$ with sufficiently small norm (see Sect. 4 hereafter). It was shown in our previous work [5] that the shape derivative of $P(\Omega)$ as in (1.1) has the form

$$
P^{\prime}(\Omega)(\theta)=\int_{\partial \Omega} u \theta \cdot \boldsymbol{n} \mathrm{d} y,
$$

where $\mathrm{d} y$ refers to the surface measure on $\partial \Omega$, and the function $u \in L^{1}(\partial \Omega)$ is given by the formula

$$
u(y)=-\int_{x \in \operatorname{ray}(y)} j^{\prime}\left(d_{\Omega}(x)\right) \prod_{1 \leq \leq n-1}\left(1+\kappa_{i}(y) d_{\Omega}(x)\right) \mathrm{d} x, \quad \forall y \in \partial \Omega .
$$

In (1.2) and (1.3), $\boldsymbol{n}$ and $\kappa_{i}(y)$ stand for the unit normal vector to $\partial \Omega$ pointing outward $\Omega$ and the $(n-1)$ principal curvatures of $\partial \Omega$ respectively; the set ray $(y)$ is the normal ray that originates from $y \in \partial \Omega$ and that stops at either the boundary $\partial D$ of the hold-all domain, or at the skeleton (or medial axis) $\Sigma$ of $\Omega$; see Section 3.1 for a short review of these notions and Figure 1A for an illustration. The normal velocity $\theta=-u n$ is then exploited by the optimization algorithm as a constraint gradient to build a descent update for the shape $\Omega$ [1]. The numerical component of this program raises the need to calculate the quantity $u: \partial \Omega \rightarrow \mathbb{R}$ defined in $(1.3)$.

In principle, the formula (1.3) featuring integration along the normal rays to $\partial \Omega$ can be implemented efficiently. In particular, its numerical evaluations at several points $y \in \partial \Omega$ can be performed in parallel. However, the practical implementation when all the functions involved in the integrand of (1.3) are discretized on a computational mesh is not trivial: it requires the computation of (i) the ray trajectories on the discrete mesh (for instance by some variant of the method of characteristics), as exemplified on Figure 1B, and (ii) the principal curvatures $\kappa_{i}$ of the boundary $\partial \Omega$ from the datum of its numerical definition. As we shall illustrate in 


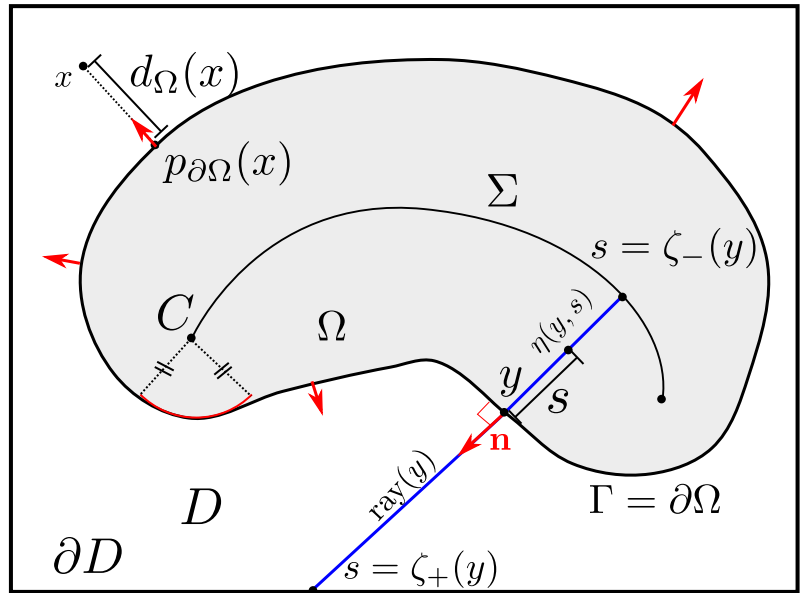

(A)

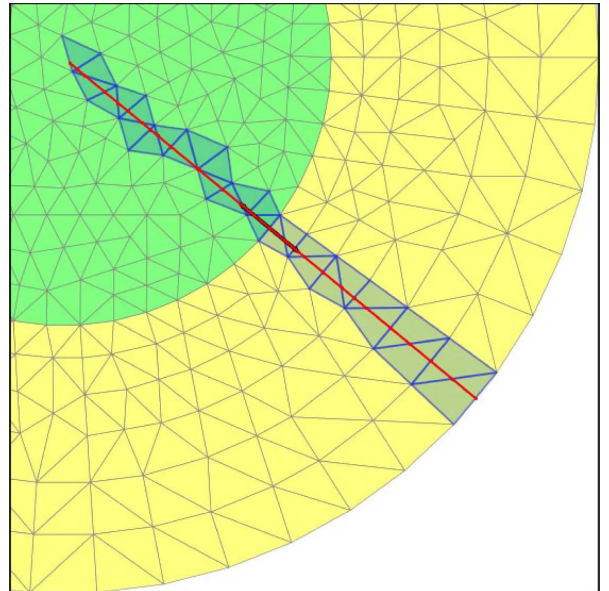

(B)

FiguRE 1. Definition of geometric quantities and integration along normal rays. (A) The shape optimization setting: shape $\Omega \subset D$, skeleton $\Sigma$ with normal rays $(\operatorname{ray}(y))_{y \in \partial \Omega}$, local coordinate change $\eta$, outward normal vector $\boldsymbol{n}$, center of curvature $C$, orthogonal projection $p_{\partial \Omega}(x)$ of a point $x \in D$ onto $\partial \Omega$. (в) Integration along rays requires the detection of the skeleton of the shape and the computation of the triangle path at the mesh level, that is the intersection points of each triangle with the ray.

our numerical investigations, the accurate calculation of these quantities - even when $\partial \Omega$ is endowed with a discretization as an explicit triangulated surface - is not trivial and is still an active research direction, already in the case of two space dimensions, and especially in the three-dimensional context $[31,49,55]$.

The objective of this paper is to introduce a robust and easy to implement method that allows to compute integral quantities such as (1.3) by solving a variational problem for advection-like operators which alleviates the need for resorting to direct integration along rays. This yields an efficient numerical method to calculate shape derivatives of geometric constraints, which is very simple to implement using a standard finite element software, and which does not raise any additional algorithmic difficulty in 3-d than in 2-d.

Our numerical method for the calculation of (1.3) is based on the following variational problem, set over a suitable Hilbert space $V_{\omega}$ :

$$
\text { Find } u \in V_{\omega} \text { such that } \forall v \in V_{\omega}, \int_{\partial \Omega} u v \mathrm{~d} s+\int_{D \backslash \bar{\Sigma}} \omega\left(\nabla d_{\Omega} \cdot \nabla u\right)\left(\nabla d_{\Omega} \cdot \nabla v\right) \mathrm{d} x=-\int_{D \backslash \bar{\Sigma}} j^{\prime}\left(d_{\Omega}\right) v \mathrm{~d} x \text {, }
$$

where $\omega>0$ is a rather arbitrary weight function (over which relevant assumptions shall be stated later on), and $\bar{\Sigma}$ is the closure of the skeleton set $\Sigma$ of $\Omega$ (see Fig. 1A and Sect. 3.1 below). Indeed, our key result is to show that (1.4) is well-posed, and that the trace of its unique solution $u$ on $\partial \Omega$ is exactly (1.3).

A formal insight that allows to see this is the following: assume that for any $v_{0} \in \mathcal{C}^{0}(\partial \Omega)$, the function $v$ satisfying $v=v_{0}$ on $\partial \Omega$ and taking constant values along the rays normal to $\partial \Omega$ (i.e. $\nabla d_{\Omega} \cdot \nabla v=0$ in $D \backslash \bar{\Sigma}$ ) 
belongs to the space $V_{\omega}$. Using $v$ as a test function in the variational formulation (1.4) then yields:

$$
\begin{aligned}
\int_{\partial \Omega} u v_{0} \mathrm{~d} s & =\int_{\partial \Omega} u v \mathrm{~d} s+\int_{D \backslash \bar{\Sigma}} \omega\left(\nabla d_{\Omega} \cdot \nabla u\right)\left(\nabla d_{\Omega} \cdot \nabla v\right) \mathrm{d} x \\
& =-\int_{D \backslash \bar{\Sigma}} j^{\prime}\left(d_{\Omega}\right) v \mathrm{~d} x=-\int_{y \in \partial \Omega}\left(\int_{z \in \operatorname{ray}(y)} j^{\prime}\left(d_{\Omega}(z)\right) \prod_{1 \leq i \leq n-1}\left(1+\kappa_{i}(y) d_{\Omega}(z)\right) \mathrm{d} s\right) v_{0}(y) \mathrm{d} y,
\end{aligned}
$$

where the last equality follows from a change of variables allowing to rewrite integration on $D \backslash \bar{\Sigma}$ as a nested integration on points $y \in \partial \Omega$ and on elements $z$ in ray $(y)$ (see (2.9) and (2.8) below). Since $v_{0} \in \mathcal{C}^{0}(\partial \Omega)$ can be chosen arbitrarily in (1.5), the identity (1.3) follows. These considerations are made rigorous in Section 2.5.

The variational formulation (1.4) makes it possible to compute integrals (1.3) along the normal rays to $\partial \Omega$ without the need to calculate these rays or the curvatures $\kappa_{i}$ explicitly on a discretization (i.e. a mesh) of the ambient space. This feature is especially convenient for shape optimization applications relying on the level set method, as described in Section 4; there, the gradient of the signed distance function $\nabla d_{\Omega}$ is easy to calculate on an unstructured mesh of the considered hold-all domain $D$ from a $\mathbb{P}_{1}$ approximation of $d_{\Omega}$. The variational formulation (1.4) can then be readily implemented in a finite element framework, even if the boundary $\Gamma=\partial \Omega$ is not meshed explicitly. Our method requires only the identification of the skeleton $\Sigma$. Although this task is notoriously difficult to carry out with a high accuracy, only a rough estimate of the location of $\Sigma$ is needed for our purpose, upon a judicious choice of the weight $\omega$ in (1.4); see the numerical examples in Section 3. Last, the previous arguments work identically when $\nabla d_{\Omega}$ is replaced with an arbitrary $\mathcal{C}^{1}$ vector field $\beta$ : we obtain that a variational formulation analogous to (1.4) (given in (2.5)) allows to compute integrals quantities along the characteristics curves of $\beta$, which is subject to offer wider applications than shape optimization.

With these perspectives in mind, the present article is organized as follows. In Section 2, we carefully discuss the mathematical setting that guarantees the existence and uniqueness of a solution to the variational problem (1.4) (in fact its generalization to arbitrary vector fields $\beta$ ), and the justification of the key identity (1.3) satisfied by the trace of its solution. For that purpose, we provide a variational theory for the advection operator $\beta \cdot \nabla$ associated to arbitrary $\mathcal{C}^{1}$ vector fields $\beta$ on the weighted graph space $V_{\omega}$; in particular, we examine the existence of traces on $\Gamma$ and we prove an adapted Poincaré inequality for functions $v \in V_{\omega}$. Let us stress that related works about these matters (e.g. $[28,32])$ usually require less regularity on the vector fields $\beta$ and do not rely on the existence of a flow $\eta$ (see (2.1) below), but at the cost of rather strong boundedness assumptions on the divergence of $\beta$ (typically $\operatorname{div}(\beta) \in L^{\infty}(D)$ ), which do not hold for our shape optimization applications (2.7) and (2.8). Indeed, in the latter situation where $\beta=\nabla d_{\Omega}$, the $\operatorname{divergence~} \operatorname{div}\left(\nabla d_{\Omega}\right)$ typically blows up near $\Sigma$; hence the need for our different approach.

In Section 3, we investigate the numerical accuracy of our variational method for calculating integrals along characteristic curves in the shape optimization setting (2.7) where $\beta=\nabla d_{\Omega}$. After a short review of the properties of the signed distance function, we compare the direct numerical integration along rays with the use of our variational method on several numerical examples where the value of (1.3) is analytically known. We also consider "practical" cases where some of the regularity assumptions imposed by our framework are not fulfilled. Most importantly, we illustrate how the selection of an appropriate weight $\omega$ in the variational formulation (1.4) allows to deal with the presence of cracks in the working domain, for instance the skeleton $\Sigma$ in shape optimization when it is not explicitly meshed.

Eventually, in Section 4, we turn to practical shape optimization applications, in the particular case of two space dimensions, in order to demonstrate the simplicity and effectiveness of our method. Let us emphasize that the variational approach (2.5) is equally simple to implement in any space dimension while the 3-d implementation of geometric integration along rays would require much more efforts than in 2-d. Elaborating on our previous works $[4,7,23]$ we demonstrate that the method proposed in this paper allows for a convenient and efficient implementation of maximum and minimum thickness constraints in structural design. 


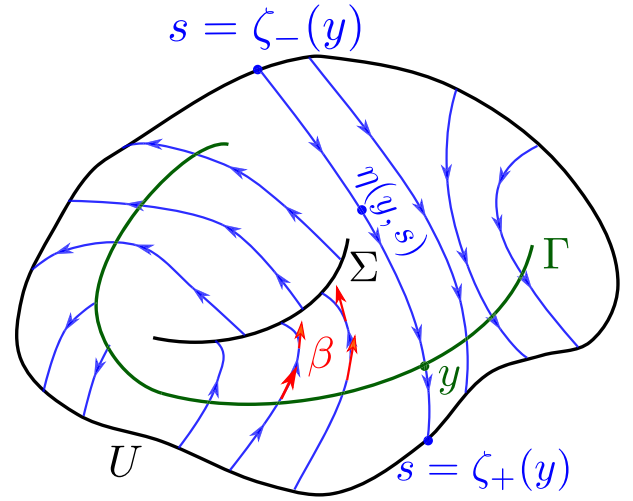

(A)

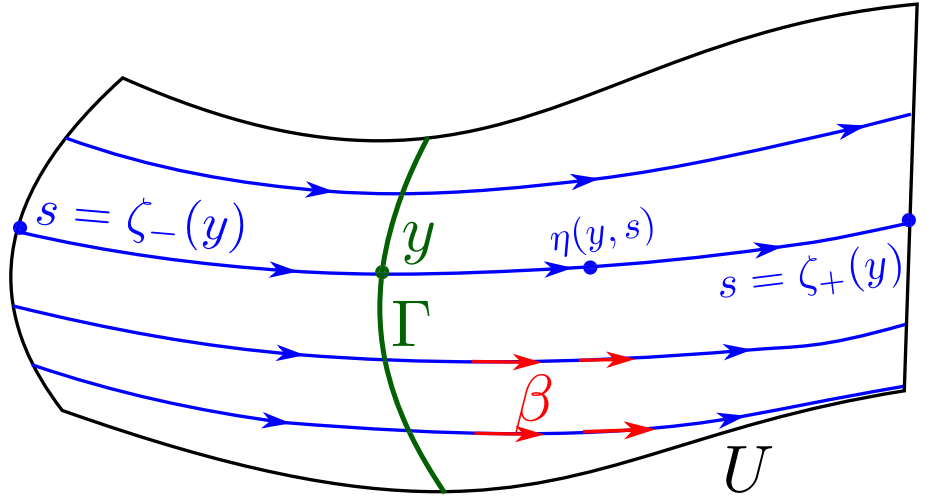

(B)

Figure 2. Two types of admissible domain $U$, with $U$-filling vector field $\beta$ and stream surface $\Gamma$, in the framework of Section 2.1. (A) Domain $U$ featuring a crack $\Sigma \subset \partial U$. (B) Smooth domain $U$.

\section{WEIGHTED GRAPH SPACE OF THE ADVECTION OPERATOR $\beta \cdot \nabla$ FOR VELOCITY FIELDS OF CLASS $\mathcal{C}^{1}$}

In this mathematical section, we consider a slightly more general variational problem than (1.4): $\nabla d_{\Omega}$ is replaced by a rather arbitrary vector field $\beta$. This setting and some technical assumptions are described in Section 2.1. In order to obtain its well-posedness and the trace identity (1.3), we introduce in Section 2.2 suitable functional spaces $V_{\omega}$, in which the directional derivative $\beta \cdot \nabla$ naturally makes sense. Then, we show in Section 2.3 that under suitable hypotheses, $\mathcal{C}^{1}$ functions are dense in $V_{\omega}$. In Section 2.4, we establish a trace theorem for functions in $V_{\omega}$ and we provide a Poincaré-type inequality. Finally, we prove in Section 2.5 that the generalized version of the variational problem (1.4) is well-posed on $V_{\omega}$ and we obtain an identity generalizing (1.3) in this context involving arbitrary fields $\beta$.

\subsection{Preliminaries, notations and assumptions}

Let $U \subset \mathbb{R}^{n}, \Gamma \subset \bar{U}$ and $\beta: U \rightarrow \mathbb{R}^{n}$ be respectively a (possibly non smooth) bounded open set, a hypersurface and a vector field of class $\mathcal{C}^{1}$. We do not require $\Gamma$ to be a compact manifold ( $\Gamma$ may differ from its closure $\bar{\Gamma}$ ); in this case, we require $\bar{\Gamma}$ to be a manifold with boundary, which in particular prevents $\Gamma$ from showing spiralling patterns near its ends - an assumption which is needed for technical reasons (see e.g. the proof of Lemma 2.13 below). Two examples of admissible settings are represented on Figure 2.

We assume that $\Gamma$ is a Poincaré section or a stream surface for $\beta$, meaning that for any $y \in \Gamma$, there is a unique characteristic curve $s \mapsto \eta(y, s)$ passing through $y=\eta(y, 0)$ at time $s=0$, and that lives in the domain $U$ on some maximal interval $s \in\left(\zeta_{-}(y), \zeta_{+}(y)\right)$. In other words, for any $y \in \Gamma,\left(\zeta_{-}(y), \zeta_{+}(y)\right)$ is the maximum existence interval such that the solution $s \mapsto \eta(y, s)$ of the ordinary differential equation

$$
\left\{\begin{array}{l}
\frac{\mathrm{d}}{\mathrm{d} s} \eta(y, s)=\beta(\eta(y, s)), \\
\eta(y, 0)=y,
\end{array}\right.
$$

remains in the domain $U$ (note that by definition, $\zeta_{-} \leq 0 \leq \zeta_{+}$). We assume that $\zeta_{-}$and $\zeta_{+}$are continuous, bounded functions on $\Gamma$, satisfying the following separation condition:

$$
\exists \epsilon>0, \forall y \in \Gamma, \zeta_{+}(y)-\zeta_{-}(y)>\epsilon .
$$


The vector field $\beta$ is required to be $U$-filling, in the sense that its related flow $\eta$ realizes a $\mathcal{C}^{1}$ diffeomorphism from the tensor product set

$$
W=\left\{(y, s) \mid y \in \Gamma, s \in\left(\zeta_{-}(y), \zeta_{+}(y)\right)\right\},
$$

onto $U$ (see [44], Chap. IV), where $W$ is a manifold obtained as an open subset of the tensor product of $\Gamma$ with the real line $\mathbb{R}$ (note that the "open" character of $W$ comes from the continuity assumption on $\zeta_{-}$and $\zeta_{+}$).

Finally, we denote by $|\mathrm{D} \eta|$ the Jacobian of the local coordinate change $\eta$ :

$$
\forall y \in \Gamma, \forall s \in\left(\zeta_{-}(y), \zeta_{+}(y)\right),|\mathrm{D} \eta|(y, s)=|\operatorname{det}(\nabla \eta)|(y, s),
$$

where the Jacobian matrix of $\eta$ reads $\nabla \eta=\left[\partial_{y} \eta \mid \partial_{s} \eta\right]$ and $\partial_{y}$ denotes the collection of derivatives with respect to the $(n-1)$ tangential coordinates of $\Gamma$.

Remark 2.1. Let us consider a particular case where $U$ is a smooth bounded domain in $\mathbb{R}^{n}$, and $\Gamma \subset \partial U$ is defined as the inlet boundary of the flow field $\beta$, i.e.

$$
\Gamma:=\{x \in \partial U \mid \beta(x) \cdot \boldsymbol{n}(x)<0\}
$$

where $\boldsymbol{n}$ is the unit normal vector to $\partial U$, pointing outward $U$, the separation condition (2.2) exactly requires that the inflow and outflow boundaries be separated by a positive minimum distance, which is a rather classical assumption in the study of the advection operator, see e.g. Section 2.1.3 in [29]. In our case, assumption (2.2) is required in the proof of Proposition 2.20.

The main point of this section is to mathematically justify that given rather arbitrary function $f: U \rightarrow \mathbb{R}$ and weight $\omega \in \mathcal{C}^{0}\left(U, \mathbb{R}_{+}^{*}\right)$, the trace of the solution $u$ to the variational problem

$$
\text { Find } u \in V_{\omega} \text { such that } \forall v \in V_{\omega}, \int_{\Gamma} u v \mathrm{~d} s+\int_{U} \omega(\beta \cdot \nabla u)(\beta \cdot \nabla v) \mathrm{d} x=\int_{U} f v \mathrm{~d} x
$$

is given explicitly in terms of line integrals by the formula

$$
\forall y \in \Gamma, u(y)=\int_{\zeta_{-}(y)}^{\zeta_{+}(y)}(f \circ \eta|\mathrm{D} \eta|)(y, s) \mathrm{d} s
$$

which yields a numerical method for computing (2.6) by solving (2.5). The shape optimization setting outlined in the introduction, which is exemplified on Figure 1A, reduces to the particular case where $\Omega \subset D$ are bounded Lipschitz domains of $\mathbb{R}^{n}$,

$$
U=D \backslash \bar{\Sigma}, \Gamma=\partial \Omega, \beta=\nabla d_{\Omega},
$$

and $\zeta_{-}(y)$ and $\zeta_{+}(y)$ are the distances at which ray $(y)$ hits either the skeleton $\Sigma$ of $\Omega$ or the boundary $\partial D$ of the hold-all domain $D$. Indeed, in this situation, the local coordinate change $\eta$ and its Jacobian $|\mathrm{D} \eta|$ are explicitly given by (see [14] and Sect. 3.1):

$$
\eta(y, s)=y+s \nabla d_{\Omega}(y),|\mathrm{D} \eta|(y, s)=\prod_{i=1}^{n-1}\left(1+\kappa_{i}(y) s\right), \quad \forall y \in \partial \Omega, \forall s \in\left(\zeta_{-}(y), \zeta_{+}(y)\right),
$$

so that the function $u$ of (1.3) coincides with the expression (2.6) for $f=-j^{\prime}\left(d_{\Omega}\right)$. Let us emphasize that, in this context, the open set $U$ is not smooth because it features a "crack", namely the skeleton $\Sigma$ (we call it a "cracked domain" in Fig. 1A); in particular, $U$ is not located on one side of its boundary. This "lack of smoothness" of $U$ prevents from using many convenient results from functional analysis [59], and thus raises the need for several technical ingredients in the sequel, which otherwise would have been fairly classical. 
Throughout the article, the considered measure on $W$ is that induced by the standard product measure of the surface measure $\mathrm{d} y$ on $\Gamma$ and of the Lebesgue measure $\mathrm{d} s$ on $\mathbb{R}$. Thus, the space $L^{1}(W)$ of integrable functions on $W$ is defined as the space of measurable functions $f: W \rightarrow \mathbb{R}$ such that $\int_{\Gamma} \int_{\zeta_{-}(y)}^{\zeta_{+}(y)}|f(y, s)| \mathrm{d} s \mathrm{~d} y<+\infty$. Integration of $f$ over $W$ is then defined by:

$$
\int_{W} f(y, s) \mathrm{d} s \mathrm{~d} y:=\int_{\Gamma} \int_{\zeta_{-}(y)}^{\zeta_{+}(y)} f(y, s) \mathrm{d} s \mathrm{~d} y .
$$

Under these notations, the classical change of variable formula between manifolds reads (see [44], Chap. XVI):

$$
\forall f \in L^{1}(U), \int_{U} f \mathrm{~d} x=\int_{W} f \circ \eta|\mathrm{D} \eta| \mathrm{d} s \mathrm{~d} y,
$$

where the Jacobian $|\mathrm{D} \eta|$ is defined by (2.4).

Let us now consider a weight function $\omega: U \rightarrow \mathbb{R}$, which will be the key ingredient of our variational formulation (2.5), satisfying the following assumptions:

(H1) $\omega \in \mathcal{C}^{0}\left(U, \mathbb{R}_{+}^{*}\right)$ is a positive, continuous function on $U$.

(H2) The transported weight $\alpha:=\omega \circ \eta|\mathrm{D} \eta| \in \mathcal{C}^{0}\left(W, \mathbb{R}_{+}^{*}\right)$ over $W$ is such that the function

$$
\begin{aligned}
g_{\alpha}: \Gamma \longrightarrow \mathbb{R}_{+} \cup\{+\infty\} \\
y \longmapsto \int_{\zeta_{-}(y)}^{\zeta_{+}(y)} \alpha(y, s) \mathrm{d} s
\end{aligned}
$$

is uniformly bounded, i.e. $g_{\alpha} \in L^{\infty}(\Gamma)$.

(H3) The transported weight $\alpha=\omega \circ \eta|\mathrm{D} \eta|$ over $W$ is such that the function

$$
\begin{aligned}
h_{\alpha}: \Gamma \longrightarrow \mathbb{R}_{+} \cup\{+\infty\} & \longrightarrow \int_{\zeta_{-}(y)}^{\zeta_{+}(y)}\left[\alpha(y, s) \int_{0}^{s} \alpha^{-1}(y, t) \mathrm{d} t\right] \mathrm{d} s
\end{aligned}
$$

is uniformly bounded: $h_{\alpha} \in L^{\infty}(\Gamma)$.

Note that, above and in the sequel, the notations $\alpha^{-1}(y, s)=1 / \alpha(y, s)$ and $\omega^{-1}(y, s)=1 / \omega(y, s)$ are used for the inverses of the scalar weights $\alpha$ and $\omega$ respectively, whereas the notation $\eta^{-1}: U \mapsto W$ shall stand for the reciprocal mapping of the diffeomorphism $\eta$.

Hypothesis (H1) is essentially a regularity assumption, positivity being no surprise for $\omega$ to be an admissible weight in the variational formulation (2.5). Notice that no assumption is made about the behavior of $\omega$ near the boundary of $U$; in particular, $\omega(x)$ may tend to 0 as $x$ approaches $\partial U$. Hypothesis (H2) is an upper-boundedness assumption for $\alpha$. The weights that we are going to consider in our practical applications in Section 3.3 will satisfy (H1) and (H2) almost automatically. Finally, (H3) is roughly a monotonicity constraint for the decay of $\alpha$ towards 0 as $s \rightarrow \zeta_{ \pm}(y)$. In practice, we will rely on the following lemma which provides a simple monotonicity condition under which the condition (H3) is fulfilled, indicating that the class of weights satisfying (H3) is large enough.

Lemma 2.2. Let $\alpha \in \mathcal{C}^{0}\left(W, \mathbb{R}_{+}^{*}\right)$ be a weight of the product form:

$$
\forall(y, s) \in W, \alpha(y, s)=f(y, s) g(y, s)
$$


where $f$ and $g$ are two positive functions on $W$ satisfying:

(i) There exist two constants $g_{-}, g_{+} \in \mathbb{R}$ such that for all $(y, s) \in W, 0<g_{-} \leq g(y, s) \leq g_{+}$.

(ii) For any $y \in \Gamma$, the real function $s \mapsto f(y, s)$ is non increasing on $\left(0, \zeta_{+}(y)\right)$ and non decreasing on $\left(\zeta_{-}(y), 0\right)$.

Then $\alpha$ satisfies the condition (H3).

Proof. Under the above conditions, it holds, for any $y \in \Gamma$ and for $s \in\left(0, \zeta_{+}(y)\right)$ :

$$
\left|\alpha(y, s) \int_{0}^{s} \alpha^{-1}(y, t) \mathrm{d} t\right| \leq \frac{g_{+}}{g_{-}} f(y, s) \int_{0}^{s} f^{-1}(y, t) \mathrm{d} t \leq \frac{g_{+}}{g_{-}} \zeta_{+}(y) \leq \frac{g_{+}}{g_{-}}\left\|\zeta_{+}-\zeta_{-}\right\|_{L^{\infty}(\Gamma)} .
$$

Arguing in the same fashion allows to prove a similar estimate when $s \in\left(\zeta_{-}(y), 0\right)$, which finally implies that $\left\|h_{\alpha}\right\|_{L^{\infty}(\Gamma)} \leq \frac{g_{+}}{g_{-}}\left\|\zeta_{+}-\zeta_{-}\right\|_{L^{\infty}(\Gamma)}^{2} ;$ this allows to conclude.

Remark 2.3. The statement of Lemma 2.2 does not require $f$ or $g$ to be continuous on $W$.

Remark 2.4. From the Liouville theorem for ordinary differential equations [18], it holds

$$
\forall(y, s) \in W,|\mathrm{D} \eta|(y, s)=\exp \left(\int_{0}^{s} \operatorname{div}(\beta) \circ \eta(y, t) \mathrm{d} t\right) .
$$

Therefore, it is straightforward to verify that (H1)-(H3) are satisfied for the constant weight $\omega=1$ whenever $\operatorname{div}(\beta) \in L^{\infty}(U)$ - a customary assumption in the study of advection operators; see e.g. [12,13, 29, 41,52].

Our setting, based on (H1)-(H3), allows to handle more general velocity fields $\beta$, with unbounded divergence, which leaves room for the Jacobian $|\mathrm{D} \eta|$ to vanish on the boundary $\partial U$. This feature is crucial to deal with the shape optimization setting (2.7); in there, the divergence $\operatorname{div}(\beta)$ of $\beta=\nabla d_{\Omega}$ blows up near centers of curvatures $C \in \bar{\Sigma}$ (see Fig. 1A and Sect. 3.1 below) where one of the principal curvatures $\kappa_{i}$ is such that $-\kappa_{i}\left(p_{\partial \Omega}(x)\right) d_{\Omega}(x) \rightarrow 1$ as $x \rightarrow C$. For instance, consider the following very simple situation where $\Omega=D$ is the unit ball in two space dimensions. Its skeleton reduces to a single point, its center. Then,

$$
\forall x \in D \backslash\{0\}, d_{\Omega}(x)=\|x\|-1, \Delta d_{\Omega}(x)=\frac{1}{\|x\|},
$$

where $\|x\|$ is the euclidean norm in $\mathbb{R}^{2}$. Therefore $\Delta d_{\Omega}(x)$ blows up at the center $x=0$ which implies $\operatorname{div}\left(\nabla d_{\Omega}\right) \notin$ $L^{\infty}(D \backslash \bar{\Sigma})$. However, in this case, (H1)-(H3) hold for $\omega=1$ : from

$$
\forall(y, s) \in \partial \Omega \times(-1,0), \eta(y, s)=(1+s) y,|\mathrm{D} \eta(y, s)|=1+s,
$$

we obtain that $\alpha(y, s)=1+s$ and $\alpha(y, s) \int_{0}^{s} \alpha^{-1}(y, t) \mathrm{d} t=(1+s) \log (1+s)$ are uniformly bounded on $W=$ $\partial \Omega \times(-1,0)$.

In the following, for a weight $\omega$ satisfying (H1), we denote by $L_{\omega}^{2}(U)$ and $L_{\alpha}^{2}(W)$ the weighted spaces

$$
L_{\omega}^{2}(U)=\left\{v \text { measurable } \mid \int_{U} \omega v^{2} \mathrm{~d} x<+\infty\right\}, \quad L_{\alpha}^{2}(W)=\left\{\widetilde{v} \text { measurable } \mid \int_{W} \alpha \tilde{v}^{2} \mathrm{~d} x<+\infty\right\},
$$

with respective corresponding $L^{2}$ norms $\|v\|_{L_{\omega}^{2}(U)}:=\left(\int_{U} \omega v^{2} \mathrm{~d} x\right)^{1 / 2}$ and $\|\widetilde{v}\|_{L_{\alpha}^{2}(W)}=\left(\int_{W} \alpha \tilde{v}^{2} \mathrm{~d} s \mathrm{~d} y\right)^{1 / 2}$. 


\subsection{Definition of the graph space $V_{\omega}$ of the advection operator $\beta \cdot \nabla$}

We recall that the assumption $\beta \in \mathcal{C}^{1}\left(U, \mathbb{R}^{n}\right)$ implies that $\operatorname{div}(\beta)$ belongs to $L_{\text {loc }}^{\infty}(U)$.

Definition 2.5 (Derivative along characteristic curves). Let $v \in L_{\text {loc }}^{1}(U)$ be a locally integrable function on $U$. The directional derivative $\beta \cdot \nabla v \in \mathcal{D}^{\prime}(U)$ is the distribution on $U$ defined by

$$
\forall \phi \in \mathcal{C}_{c}^{\infty}(U), \int_{U}(\beta \cdot \nabla v) \phi \mathrm{d} x=\int_{U}(-(\beta \cdot \nabla \phi) v-\operatorname{div}(\beta) \phi v) \mathrm{d} x .
$$

Remark 2.6. The definition (2.15) of $\beta \cdot \nabla$ mimics the integration by part formula that holds for functions $v$ of class $\mathcal{C}^{1}$. Considering test functions $\phi \in \mathcal{C}_{c}^{\infty}(U)$ allows to avoid imposing classical regularity requirements on the open domain $U$ such as that being locally located on one side of its boundary [59].

Definition 2.7 (Graph space of the operator $\beta \cdot \nabla)$. Let $\omega \in \mathcal{C}^{0}\left(U, \mathbb{R}_{+}^{*}\right)$ be a positive weight on $U$. The weighted graph space $V_{\omega}$ of the advection operator $\beta \cdot \nabla$ is defined by:

$$
V_{\omega}=\left\{v \in L_{\omega}^{2}(U) \mid \beta \cdot \nabla v \in L_{\omega}^{2}(U)\right\} .
$$

$V_{\omega}$ is a Hilbert space when it is endowed with the norm

$$
\|v\|_{V_{\omega}}:=\left(\int_{U} \omega v^{2} \mathrm{~d} x+\int_{U} \omega|\beta \cdot \nabla v|^{2} \mathrm{~d} x\right)^{1 / 2} .
$$

Remark 2.8. The completeness of $V_{\omega}$ follows from very standard arguments, see e.g. [29,60]. It is also easy to see from the assumption $\omega \in \mathcal{C}^{0}\left(U, \mathbb{R}_{+}^{*}\right)$ that the inclusion $L_{\omega}^{2}(U) \subset L_{\text {loc }}^{1}(U)$ holds.

Let us then introduce corresponding notions of derivative with respect to the $s$ variable and of graph space on the set $W$.

Definition 2.9 (Graph space of the derivative $\left.\partial_{s}\right)$. Let $\alpha \in \mathcal{C}^{0}\left(W, \mathbb{R}_{+}^{*}\right)$ be a positive weight on $W$. The weak derivative $\partial_{s} \tilde{v} \in \mathcal{D}^{\prime}(W)$ of a function $\tilde{v} \in L_{\text {loc }}^{1}(W)$ is the distribution defined by

$$
\forall \phi \in \mathcal{C}_{c}^{\infty}(W), \int_{W} \partial_{s} \tilde{v} \phi \mathrm{d} y \mathrm{~d} s=-\int_{W} \tilde{v} \partial_{s} \phi \mathrm{d} y \mathrm{~d} s .
$$

The weighted graph space of the operator $\partial_{s}$ is defined by:

$$
\widetilde{V}_{\alpha}=\left\{\tilde{v} \in L_{\alpha}^{2}(W) \mid \partial_{s} \tilde{v} \in L_{\alpha}^{2}(W)\right\} .
$$

This space is a Hilbert space when it is equipped with the norm

$$
\|\tilde{v}\|_{\widetilde{V}_{\alpha}}:=\left(\int_{W} \alpha \tilde{v}^{2} \mathrm{~d} s \mathrm{~d} y+\int_{W} \alpha\left|\partial_{s} \tilde{v}\right|^{2} \mathrm{~d} s \mathrm{~d} y\right)^{1 / 2} .
$$

Remark 2.10. The defining identity (2.15) of the weak derivative $\beta \cdot \nabla v$ also holds for test functions $\phi \in$ $C_{c}^{1}(U)$, as follows from a standard density argument. Likewise, (2.17) holds for test functions $\phi$ which are only continuously differentiable with respect to the $s$ variable.

The motivation behind the introduction of the spaces $\widetilde{V}_{\alpha}$ is that they allow to transfer the difficulty of studying the "curvilinear" directional derivative $\beta \cdot \nabla$ over the domain $U$, to that of the more standard, "flat" derivative $\partial_{s}$ over $W$. The price to pay is the need to account for the weight of the Jacobian $|\mathrm{D} \eta|$ resulting from the change of variables. This point is made precise by the following technical lemma. 
Lemma 2.11. Let $\omega \in \mathcal{C}^{0}\left(U, \mathbb{R}_{+}^{*}\right)$ be a positive weight on $U$, and let $\alpha=\omega \circ \eta|\mathrm{D} \eta|$. The following equivalence holds true:

$$
\in V_{\omega} \Leftrightarrow v \circ \eta \in \widetilde{V}_{\alpha}
$$

Additionally, for any $v \in V_{\omega}$, the function $\tilde{v}=v \circ \eta$ satisfies:

(i) $\partial_{s} \tilde{v}=(\beta \cdot \nabla v) \circ \eta$,

(ii) $\|v\|_{V_{\omega}}=\|v \circ \eta\|_{\widetilde{V}_{\alpha}}$.

Proof. Let $v \in V_{\omega}$; it follows from the change of variables (2.9) that $\tilde{v}:=v \circ \eta$ belongs to $L_{\alpha}^{2}(W)$. Moreover, we observe that $\tilde{v}$ has a weak derivative $\partial_{s} \tilde{v}$ given by:

$$
\partial_{s} \tilde{v}=(\beta \cdot \nabla v) \circ \eta
$$

Indeed, using the definition (2.15) of the weak derivative along $\beta$ with a test function $\phi \in \mathcal{C}_{c}^{1}(U)$ of the form $\phi=\tilde{\phi} \circ \eta^{-1}$ for an arbitrary $\tilde{\phi} \in \mathcal{C}_{c}^{1}(W)$ (see Rem. 2.10), then using (2.9), we obtain:

$$
\forall \tilde{\phi} \in \mathcal{C}_{c}^{1}(W), \int_{W}(\beta \cdot \nabla v) \circ \eta \tilde{\phi}|\mathrm{D} \eta| \mathrm{d} s \mathrm{~d} y=\int_{W}\left(-\partial_{s} \tilde{\phi} \tilde{v}-\operatorname{div}(\beta) \circ \eta \tilde{\phi} \tilde{v}\right)|\mathrm{D} \eta| \mathrm{d} s \mathrm{~d} y
$$

where we have used the equality $\partial_{s} \tilde{\phi}=(\beta \cdot \nabla \phi) \circ \eta$. Now using that $|\mathrm{D} \eta|$ is continuously differentiable with respect to $s$ with $\partial_{s}|\mathrm{D} \eta|=\operatorname{div}(\beta) \circ \eta|\mathrm{D} \eta|$, as a consequence of the Liouville formula (2.13), (2.20) rewrites:

$$
\forall \tilde{\phi} \in \mathcal{C}_{c}^{1}(W), \int_{W}(\beta \cdot \nabla v) \circ \eta \tilde{\phi}|\mathrm{D} \eta| \mathrm{d} s \mathrm{~d} y=\int_{W}\left(-\partial_{s} \tilde{\phi} \tilde{v}|\mathrm{D} \eta|-\partial_{s}|\mathrm{D} \eta| \tilde{\phi} \tilde{v}\right) \mathrm{d} s \mathrm{~d} y=-\int_{W} \tilde{v} \partial_{s}(\tilde{\phi}|\mathrm{D} \eta|) \mathrm{d} s \mathrm{~d} y .
$$

By a standard density argument, the above equality holds more generally for functions $\tilde{\phi}$ that are only continuously differentiable with respect to $s$ on $W$. Therefore, taking $\tilde{\phi}=\tilde{\psi} /|\mathrm{D} \eta|$ as a test function in the above equation for arbitrary $\tilde{\psi} \in \mathcal{C}_{c}^{1}(W)$ yields (2.19). The change of variables (2.9) now yields $\|v\|_{V_{\omega}}=\|v \circ \eta\|_{\widetilde{V}_{\alpha}}$, and so $v \circ \eta \in \widetilde{V}_{\alpha}$.

Conversely, one proves in a similar way that if $\tilde{v}$ is in $\widetilde{V}_{\alpha}$, the function $v=\tilde{v} \circ \eta^{-1}$ belongs to $V_{\omega}$, which terminates the proof.

Remark 2.12. In the following we prove a density result, a trace theorem and a Poincaré inequality in $\widetilde{V}_{\alpha}$ and we then obtain the direct counterparts of these results in the setting of the graph space $V_{\omega}$ thanks to the above Lemma 2.11. There exists actually a wide literature about weighted Sobolev spaces such as $\widetilde{V}_{\alpha}[33,42,43,60]$, in which analogous results are proved for weights $\alpha$ in the so-called Mückenhoupt class $A_{2}$ (see e.g. [30] for a definition of the class $A_{p}$ ). Our working assumptions however are of a different nature: for instance, the hypothesis (H3) essentially requires that the inverse weight $\alpha^{-1}$ belong to the Mückenhoupt class $A_{1}$.

\subsection{Density of functions of class $\mathcal{C}^{1}$ in the weighted space $V_{\omega}$}

We now examine the density of $\mathcal{C}^{\infty}(W) \cap \widetilde{V}_{\alpha}$ in $\widetilde{V}_{\alpha}$, whence we shall infer the density of $\mathcal{C}^{1}(U) \cap V_{\omega}$ in $V_{\omega}$ - the loss of regularity between both statements coming from the fact that the coordinate change $\eta$ is only of class $\mathcal{C}^{1}$ on $W$.

Our study classically involves mollifying functions; since the space $\widetilde{V}_{\alpha}$ of interest contains functions defined on the manifold $W$, a little treatment is in order. In particular, we shall need the so-called Ahlfors regularity of $\Gamma$ (see [24]); this is the purpose of the next lemma, whose proof is outlined for the convenience of the reader. Here and throughout the article, $B(y, h) \subset \mathbb{R}^{n}$ stands for the open ball with center $y$ and radius $h$. The measure of a Lebesgue measurable set $A \subset \mathbb{R}^{n}$ is denoted by $|A|$. 
Lemma 2.13 (Area covered by extrinsic balls on an embedded manifold). Let $\Gamma \subset \mathbb{R}^{n}$ be a $\mathcal{C}^{1}$ hypersurface such that either $\Gamma$ is compact $(\Gamma=\bar{\Gamma})$ or $\bar{\Gamma}$ is a compact manifold with boundary. Then there exists $h_{0}>0$ and constants $m>0$ and $M>0$ depending only on $\Gamma$ such that for any $0<h<h_{0}$,

$$
\forall y \in \Gamma, m h^{n-1} \leq|\Gamma \cap B(y, h)| \leq M h^{n-1} .
$$

Proof. Since $\bar{\Gamma}$ is compact, there exist finitely many open subset $V_{i} \subset \mathbb{R}^{n}, i=1, \ldots, N$, such that $\bar{\Gamma} \subset \bigcup_{i} V_{i}$, and for each $i=1, \ldots, N$, there exists a local coordinate chart $\phi_{i}: U_{i} \subset \mathbb{R}^{n-1} \rightarrow \phi_{i}\left(U_{i}\right) \subset \bar{\Gamma}$ such that $\phi_{i}\left(U_{i}\right)=V_{i} \cap \bar{\Gamma}$. The set $U_{i}$ is a convex open subset of $\mathbb{R}^{n-1}$ if $V_{i} \cap \partial \Gamma=\emptyset$; when the latter intersection is not empty, $U_{i}$ is of the form $U_{i}=\widetilde{U}_{i} \cap \mathbb{R}_{+}^{n-1}$, where $\widetilde{U}_{i} \subset \mathbb{R}^{n-1}$ is a convex open subset, and $\mathbb{R}_{+}^{n-1}$ is the upper half-space of $\mathbb{R}^{n-1}$. Let $h_{0}>0$ be a Lebesgue's number associated with this cover, that is:

$$
\forall y \in \Gamma, \exists i \in\{1, \ldots, N\}, B\left(y, h_{0}\right) \subset \subset V_{i} .
$$

Now, given $y \in V_{i}$, one has for any $0<h<h_{0}$ :

$$
|\Gamma \cap B(y, h)|=\int_{\Gamma} \mathbf{1}_{B(y, h)} \mathrm{d} z=\int_{\phi_{i}\left(U_{i}\right)} \mathbf{1}_{B(y, h)} \mathrm{d} z=\int_{U_{i}} \mathbf{1}_{\phi_{i}^{-1}(B(y, h))}\left|\mathrm{D} \phi_{i}\right| \mathrm{d} x,
$$

where $i$ is the index supplied by (2.22) and $\mathbf{1}_{A}$ denotes the characteristic function of a subset $A \subset \mathbb{R}^{n}$, and $\left|\mathrm{D} \phi_{i}\right|$ is the Jacobian associated to the change of variables between manifolds induced by $\phi_{i}$.

Let $\sigma_{n-1}\left(\nabla \phi_{i}(x)\right) \leq \sigma_{1}\left(\nabla \phi_{i}(x)\right)$ be respectively the smallest and the largest singular values of the $n \times(n-1)$ Jacobian matrix $\nabla \phi_{i}(x)$, and let $0<\sigma_{-} \leq \sigma_{+}$be defined by:

$$
\sigma_{-}=\min _{i=1, \ldots, N} \inf _{x \in U_{i}} \sigma_{n-1}\left(\nabla \phi_{i}(x)\right), \sigma_{+}=\max _{i=1, \ldots, \ldots N} \sup _{x \in U_{i}} \sigma_{1}\left(\nabla \phi_{i}(x)\right) .
$$

Applying the Taylor formula to $\phi_{i}$ yields:

$$
\forall i=1, \ldots, N, \forall\left(x_{0}, x_{1}\right) \in U_{i}, \sigma_{-}\left\|x_{1}-x_{0}\right\| \leq\left\|\phi_{i}\left(x_{1}\right)-\phi_{i}\left(x_{0}\right)\right\| \leq \sigma_{+}\left\|x_{1}-x_{0}\right\| .
$$

Therefore, possibly shrinking the value of the constant $h_{0}$ supplied by $(2.22)$ and taking $x_{0}=\phi_{i}^{-1}(y)$, we obtain:

$$
\forall 0<h<h_{0}, B\left(x_{0}, \frac{h}{\sigma_{+}}\right) \subset \phi_{i}^{-1}(B(y, h)) \subset B\left(x_{0}, \frac{h}{\sigma_{-}}\right) \subset U_{i} .
$$

Finally, denoting by $\left|B_{n-1}\right|$ the volume of the unit ball in $\mathbb{R}^{n-1}$, we obtain:

$$
\begin{aligned}
\frac{1}{2}\left|B_{n-1}\right|\left(\frac{\sigma_{-}}{\sigma_{+}}\right)^{n-1} h^{n-1} & \leq \int_{U_{i}} \mathbf{1}_{B\left(x_{0}, h / \sigma_{+}\right)}\left|\mathrm{D} \phi_{i}\right| \mathrm{d} x \\
& \leq \int_{U_{i}} \mathbf{1}_{\phi_{i}^{-1}(B(y, h))}\left|\mathrm{D} \phi_{i}\right| \mathrm{d} x=|\Gamma \cap B(y, h)| \\
& \leq \int_{U_{i}} \mathbf{1}_{B\left(x_{0}, h / \sigma_{-}\right)}\left|\mathrm{D} \phi_{i}\right| \mathrm{d} x \leq\left|B_{n-1}\right|\left(\frac{\sigma_{+}}{\sigma_{-}}\right)^{n-1} h^{n-1},
\end{aligned}
$$

which completes the proof.

In what follows, the hypersurface $\Gamma$ is the one introduced by Section 2.1. In the next lemma, we construct the kernels $\rho_{h}$ and $\xi_{h}$ which shall be used for mollification purposes in $W$. 
Lemma 2.14. For any $h>0$, there exist two positive, smooth functions $\rho_{h} \in \mathcal{C}_{c}^{\infty}\left(\mathbb{R}^{n} \times \mathbb{R}^{n}, \mathbb{R}\right)$ and $\xi_{h} \in$ $\mathcal{C}_{c}^{\infty}(\mathbb{R}, \mathbb{R})$ satisfying the conditions:

(i) For all points $y \in \mathbb{R}^{n}, \operatorname{supp}\left(\rho_{h}(y, \cdot)\right) \subset B(y, h)$.

(ii) For any $y \in \mathbb{R}^{n}, \int_{\Gamma} \rho_{h}(y, z) \mathrm{d} z=1$.

(iii) There exist constants $C>0$ and $h_{0}>0$ depending only on $\Gamma$ such that

$$
\forall 0<h<h_{0}, \forall z \in \Gamma, \int_{\Gamma} \rho_{h}(y, z) \mathrm{d} y \leq C .
$$

(iv) $\operatorname{supp}\left(\xi_{h}\right) \subset[-h, h]$ and $\int_{\mathbb{R}} \xi_{h}(s) \mathrm{d} s=1$.

Proof. Let $\rho \in \mathcal{C}_{c}^{\infty}\left(\mathbb{R}^{n}, \mathbb{R}\right)$ be a smooth, positive function such that $\operatorname{supp}(\rho) \subset B(0,1), \rho \leq 1$ on $\mathbb{R}^{n}$ and $\rho_{\mid B(0,1 / 2)}=1$; we define $\rho_{h}$ by:

$$
\forall h>0, \forall(y, z) \in \mathbb{R}^{n} \times \mathbb{R}^{n}, \rho_{h}(y, z):=\frac{\rho\left(\frac{y-z}{h}\right)}{\int_{\Gamma} \rho\left(\frac{y-z}{h}\right) \mathrm{d} z} ;
$$

note that $\rho_{h}(y, z)$ is not a function of $(y-z)$. The conditions (i) and (ii) of the statement are obviously satisfied by (2.24). The condition (iii) is a consequence of Lemma 2.13, which implies:

$$
\begin{aligned}
& \forall 0<h<h_{0}, \forall y \in \Gamma, \int_{\Gamma} \rho\left(\frac{y-z}{h}\right) \mathrm{d} z \geq\left|\Gamma \cap B\left(y, \frac{h}{2}\right)\right| \geq m\left(\frac{h}{2}\right)^{n-1}, \\
& \forall 0<h<h_{0}, \forall z \in \Gamma, \int_{\Gamma} \rho\left(\frac{y-z}{h}\right) \mathrm{d} y \leq|\Gamma \cap B(z, h)| \leq M h^{n-1},
\end{aligned}
$$

so that (2.23) holds with $C=2^{n-1} M / m$.

Eventually, a function $\xi_{h}$ satisfying (iv) is constructed from any positive function $\xi \in \mathcal{C}_{c}^{\infty}(\mathbb{R}, \mathbb{R})$ with compact support inside $[-1,1]$ and unit integral over $\mathbb{R}$, by setting $\xi_{h}=h^{-1} \xi(\cdot / h)$.

Definition 2.15 (Mollification on the tensor product manifold $W$ ). For $h>0$, let $\rho_{h}$ and $\xi_{h}$ be two functions as in the statement of Lemma 2.14. For any $u \in L_{\mathrm{loc}}^{1}(W),(y, s) \in W$ and $h>0$ sufficiently small (depending on $(y, s))$, the mollification of $u$ is the function $u_{h}=\rho_{h} \xi_{h} * u$ defined by

$$
\left(\rho_{h} \xi_{h} * u\right)(y, s)=\int_{\Gamma} \int_{\mathbb{R}} \rho_{h}(y, z) \xi_{h}(s-t) u(z, t) \mathrm{d} t \mathrm{~d} z .
$$

Note that for a given open subdomain $W_{1} \subset \subset W$ and because $\bar{\Gamma}$ is compact, there exists $h_{0}>0$, depending on $W_{1}$, sufficiently small so that $(2.25)$ makes sense for any $(y, s) \in W_{1}$ and $0<h<h_{0}$.

Lemma 2.16. The following properties hold true:

(i) If $u \in L_{\text {loc }}^{1}(W)$, for any subdomain $W_{1} \subset \subset W$ and for $h>0$ sufficiently small, the convolution $\rho_{h} \xi_{h} * u$ is of class $\mathcal{C}^{\infty}$ on $W_{1}$.

(ii) If $u \in \widetilde{V}_{\alpha}$, then for any subdomain $W_{1} \subset \subset W$ and for $h$ sufficiently small, $\partial_{s}\left(\rho_{h} \xi_{h} * u\right)=\rho_{h} \xi_{h} * \partial_{s} u$.

(iii) If $\phi \in \mathcal{C}^{0}(W, \mathbb{R})$ then $\rho_{h} \xi_{h} * \phi \rightarrow \phi$ in $L_{\mathrm{loc}}^{\infty}(W)$ as $h \rightarrow 0$.

Proof. (i) This results from the Lebesgue dominated convergence theorem and the smoothness of $\rho_{h}$ and $\xi_{h}$. 
(ii) This is again a consequence of the Lebesgue dominated theorem and of an integration by parts:

$$
\begin{aligned}
\forall(y, s) \in W_{1},\left(\rho_{h} \xi_{h} * \partial_{s} u\right)(y, s) & =\int_{\Gamma} \int_{\mathbb{R}} \rho_{h}(y, z) \xi_{h}(s-t) \partial_{s} u(z, t) \mathrm{d} t \mathrm{~d} z \\
& =\int_{\Gamma} \int_{\mathbb{R}} \rho_{h}(y, z) \partial_{s} \xi_{h}(s-t) u(z, t) \mathrm{d} t \mathrm{~d} z=\partial_{s}\left(\rho_{h} \xi_{h} * u\right) .
\end{aligned}
$$

(iii) For a given subset $W_{1} \subset \subset W$, let $\epsilon>0$ and $h_{1}>0$ be uniform continuity constants for $\phi$ on $W_{1}$, i.e.

$$
\forall 0<h<h_{1},(y, s) \in W_{1},(z, t) \in W_{1},(|| y-z||<h \text { and }|s-t|<h) \Rightarrow|\phi(z, t)-\phi(y, s)|<\epsilon .
$$

Then for any $0<h<h_{1}$ :

$$
\begin{aligned}
\forall(y, s) \in W_{1},\left|\rho_{h} \xi_{h} * \phi(y, s)-\phi(y, s)\right| & =\left|\int_{\Gamma} \int_{\mathbb{R}} \rho_{h}(y, z) \xi_{h}(s-t)(\phi(z, t)-\phi(y, s)) \mathrm{d} t \mathrm{~d} z\right| \\
& \leq \epsilon \int_{\Gamma} \int_{\mathbb{R}} \rho_{h}(y, z) \xi_{h}(s-t) \mathrm{d} t \mathrm{~d} z=\epsilon .
\end{aligned}
$$

Proposition 2.17. Let $\alpha \in \mathcal{C}^{0}\left(W, \mathbb{R}_{+}^{*}\right)$. Then for any function $u \in L_{\alpha, \text { loc }}^{2}(W), u_{h}:=\rho_{h} \xi_{h} * u$ belongs to $L_{\alpha, \text { loc }}^{2}(W) \cap \mathcal{C}^{\infty}(W)$ and $u_{h} \rightarrow u$ in $L_{\alpha, \text { loc }}^{2}(W)$.

Proof. We adapt the proof of Proposition 1.18, page 30 in [53]. Let $W_{1} \subset \subset W$ be an open subset of $W$ and consider another open subset $W_{2}$ such that $W_{1} \subset \subset W_{2} \subset \subset W$. For $\epsilon>0$, let $\phi \in \mathcal{C}^{0}(W)$ be such that (see [54]):

$$
\|u-\phi\|_{L_{\alpha}^{2}\left(W_{2}\right)}<\epsilon .
$$

From Lemma 2.16, $\rho_{h} \xi_{h} * \phi \rightarrow \phi$ in $L^{\infty}\left(W_{1}\right)$ as $h \rightarrow 0$ and so, for $h$ small enough, $\left\|\rho_{h} \xi_{h} * \phi-\phi\right\|_{L_{\alpha}^{2}\left(W_{1}\right)} \leq \epsilon$. Then,

$$
\left\|u-u_{h}\right\|_{L_{\alpha}^{2}\left(W_{1}\right)} \leq\|u-\phi\|_{L_{\alpha}^{2}\left(W_{1}\right)}+\left\|\phi-\rho_{h} \xi_{h} * \phi\right\|_{L_{\alpha}^{2}\left(W_{1}\right)}+\left\|\rho_{h} \xi_{h} * \phi-\rho_{h} \xi_{h} * u\right\|_{L_{\alpha}^{2}\left(W_{1}\right)} .
$$

The first two terms in the right-hand side of (2.27) are controlled by $\epsilon$ owing to the previous discussion; as for the last term, we get from the Cauchy-Schwarz inequality:

$$
\begin{aligned}
\forall(y, s) \in W_{1},\left|\rho_{h} \xi_{h} *(\phi-u)(y, s)\right|^{2} & =\left|\int_{\Gamma} \int_{\mathbb{R}} \rho_{h}(y, z) \xi_{h}(s-t)(\phi(z, t)-u(z, t)) \mathrm{d} t \mathrm{~d} z\right|^{2} \\
\leq & \left(\int_{\Gamma} \int_{\mathbb{R}} \rho_{h}(y, z) \xi_{h}(s-t) \alpha^{-1}(z, t) \mathrm{d} t \mathrm{~d} z\right) \\
& \times\left(\int_{\Gamma} \int_{\mathbb{R}} \rho_{h}(y, z) \xi_{h}(s-t) \alpha(z, t)|\phi(z, t)-u(z, t)|^{2} \mathrm{~d} t \mathrm{~d} z\right) .
\end{aligned}
$$


Multiplying both sides by $\alpha$ and integrating over $W_{1}$ yields:

$$
\begin{aligned}
\left\|\rho_{h} \xi_{h} *(\phi-u)\right\|_{L_{\alpha}^{2}\left(W_{1}\right)}^{2} \leq & \left\|\alpha\left(\rho_{h} \xi_{h} * \alpha^{-1}\right)\right\|_{L^{\infty}\left(W_{1}\right)} \\
& \times \int_{(y, s) \in W_{1}}\left(\int_{\Gamma} \int_{\mathbb{R}} \rho_{h}(y, z) \xi_{h}(s-t) \alpha(z, t)|\phi(z, t)-u(z, t)|^{2} \mathrm{~d} t \mathrm{~d} z\right) \mathrm{d} s \mathrm{~d} y \\
\leq & C\left\|\alpha\left(\rho_{h} \xi_{h} * \alpha^{-1}\right)\right\|_{L^{\infty}\left(W_{1}\right)}\|\phi-u\|_{L_{\alpha}^{2}\left(W_{2}\right)}^{2},
\end{aligned}
$$

where $C$ is the constant supplied by the condition (iii) in the statement of Lemma 2.14. By assumption, $\alpha^{-1}$ is a continuous function on $W_{2}$, which implies by Lemma 2.16 that $\alpha\left(\rho_{h} \xi_{h} * \alpha^{-1}\right) \rightarrow 1$ in $L^{\infty}\left(W_{1}\right)$. In particular, $\left\|\alpha\left(\rho_{h} \xi_{h} * \alpha^{-1}\right)\right\|_{L^{\infty}\left(W_{1}\right)}$ is bounded by some constant $C^{\prime}$. Finally, using (2.26), we obtain from (2.27) that for $h>0$ small enough:

$$
\left\|u-u_{h}\right\|_{L_{\alpha}^{2}\left(W_{1}\right)} \leq\left(C C^{\prime}+2\right) \epsilon
$$

which is the desired result.

We conclude this subsection with the desired density result of $\mathcal{C}^{1}$ functions in $V_{\omega}$ :

Corollary 2.18. (i) Let $\alpha \in \mathcal{C}^{0}\left(W, \mathbb{R}_{+}^{*}\right)$ be a positive weight on $W$; the space $\mathcal{C}^{\infty}(W) \cap \widetilde{V}_{\alpha}$ is dense in $\widetilde{V}_{\alpha}$.

(ii) Let $\omega \in \mathcal{C}^{0}\left(U, \mathbb{R}_{+}^{*}\right)$ be a positive weight on $U$; the space $\mathcal{C}^{1}(U) \cap V_{\omega}$ is dense in $V_{\omega}$.

Proof. The proof of the density (i) of $\mathcal{C}^{\infty}(W) \cap \widetilde{V}_{\alpha}$ in $\widetilde{V}_{\alpha}$ relies on a partition of unity argument and on the properties of Lemma 2.16 and proposition 2.17, exactly along the lines of the proof of Theorem 5.15 in [53], to which the reader is referred for details.

The density (ii) of $\mathcal{C}^{1}(U)$ in $V_{\omega}$ follows then from the density of $\mathcal{C}^{\infty}(W) \cap \widetilde{V}_{\alpha}$ in $\widetilde{V}_{\alpha}$ with $\alpha=\omega \circ \eta|\mathrm{D} \eta|$ and by composition with the $\mathcal{C}^{1}$ diffeomorphism $\eta$.

Remark 2.19. Corollary 2.18 does not imply the density of $\mathcal{C}^{1}(\bar{U})$ in $V_{\omega}$. A result of this kind would require careful regularity assumptions on $\partial U$ and on the behavior of $\omega$ near $\partial U$.

\subsection{Trace theorem and Poincaré inequality in $V_{\omega}$}

In this section, the trace operator on $\Gamma$ is defined and studied for functions in the weighted space $V_{\omega}$, or equivalently for functions in $\widetilde{V}_{\alpha}$ on $\Gamma \times\{0\}=\{(y, 0) \mid y \in \Gamma\}$. In the sequel, with a little abuse of notations, the latter set $\Gamma \times\{0\}$ is identified with $\Gamma$.

Proposition 2.20 (Trace theorem). Let $\omega \in \mathcal{C}^{0}\left(U, \mathbb{R}_{+}^{*}\right)$ be a positive weight on $U$. The trace operator

$$
\begin{aligned}
\gamma: \mathcal{C}^{1}(U) & \rightarrow L^{2}(\Gamma) \\
v & \mapsto \gamma(v)=v_{\mid \Gamma}
\end{aligned}
$$

induces a bounded operator $V_{\omega} \rightarrow L^{2}(\Gamma)$; there exists a constant $C>0$ (possibly depending on the weight $\omega$ ) such that

$$
\forall v \in V_{\omega},\|\gamma(v)\|_{L^{2}(\Gamma)} \leq C\|v\|_{V_{\omega}} .
$$

Proof. Introducing $\alpha=\omega \circ \eta|\mathrm{D} \eta|$, using the change of variables (2.9) and the density result of Corollary 2.18, it is enough to prove that there exists a constant $C>0$ such that:

$$
\forall \tilde{v} \in \mathcal{C}^{\infty}(W) \cap \widetilde{V}_{\alpha},\|\gamma(\tilde{v})\|_{L^{2}(\Gamma)} \leq C\|\tilde{v}\|_{\widetilde{V}_{\alpha}}
$$

Let us consider the following partition of $\Gamma: \Gamma=\Gamma_{+} \cup \Gamma_{-}$, where $\Gamma_{+}=\left\{y \in \Gamma \mid \zeta_{-}(y) \geq-\epsilon / 2\right\}, \Gamma_{-}=\Gamma \backslash \Gamma_{+}$, and $\epsilon$ is the parameter featured in the separation condition (2.2). Then

$$
\begin{aligned}
& \forall y \in \Gamma_{+}, 0<\epsilon / 2<\zeta_{+}(y), \\
& \forall y \in \Gamma_{-}, \zeta_{-}(y)<-\epsilon / 2<0 .
\end{aligned}
$$


Now, let $a=\epsilon / 2$ and $\xi \in \mathcal{C}_{c}^{\infty}(\mathbb{R})$ be such that $\xi(0)=1$ and $\xi(-a)=\xi(a)=0$. Let $K$ be the bounded measurable set defined by $K:=\left(\Gamma_{-} \times[-a, 0]\right) \cup\left(\Gamma_{+} \times[0, a]\right) \subset W$. Then for any function $\tilde{v} \in \mathcal{C}^{\infty}(W) \cap \widetilde{V}_{\alpha}$, it holds:

$$
\begin{aligned}
\int_{\Gamma}|\tilde{v}(y, 0)|^{2} \mathrm{~d} y & =\int_{\Gamma}\left|\tilde{v}(y, 0)^{2} \xi(0)\right| \mathrm{d} y=\int_{\Gamma_{-}} \int_{-a}^{0} \partial_{s}\left(\tilde{v}^{2} \xi\right) \mathrm{d} s \mathrm{~d} y+\int_{\Gamma_{+}} \int_{a}^{0} \partial_{s}\left(\tilde{v}^{2} \xi\right) \mathrm{d} s \mathrm{~d} y \\
& \leq \int_{K}\left|2 \tilde{v} \partial_{s} \tilde{v} \xi+\tilde{v}^{2} \partial_{s} \xi\right| \mathrm{d} s \mathrm{~d} y \\
& \leq 2\left\|\alpha^{-1} \xi\right\|_{L^{\infty}(K)}\|\tilde{v}\|_{L_{\alpha}^{2}(W)}\left\|\partial_{s} \tilde{v}\right\|_{L_{\alpha}^{2}(W)}+\left\|\alpha^{-1} \partial_{s} \xi\right\|_{L^{\infty}(K)}\|\tilde{v}\|_{L_{\alpha}^{2}(W)}^{2} \\
& \leq\left(2\left\|\alpha^{-1} \xi\right\|_{L^{\infty}(K)}+\left\|\alpha^{-1} \partial_{s} \xi\right\|_{L^{\infty}(K)}\right)\|\tilde{v}\|_{\widetilde{V}_{\alpha}}^{2}
\end{aligned}
$$

which implies (2.30) and therefore terminates the proof of Proposition 2.20.

Remark 2.21. The proof of Proposition 2.20 supplies the existence of the trace on $\Gamma$ of an arbitrary function $\tilde{v} \in \widetilde{V}_{\alpha}$, which we shall also denote by $\tilde{v}_{\mid \Gamma}$.

For later purposes (see Sect. 2.5), we shall need the surjectivity of the above trace operator; this is the purpose of the next proposition.

Proposition 2.22 (Surjectivity of traces). Let $\omega \in \mathcal{C}^{0}\left(U, \mathbb{R}_{+}^{*}\right)$ be a positive continuous weight.

(i) The trace operator defined by $(2.28)$ is surjective from $V_{\omega}$ onto $L^{2}(\Gamma)$ :

$$
L^{2}(\Gamma)=\left\{v_{\mid \Gamma} \mid v \in V_{\omega}\right\} .
$$

(ii) If $\omega$ additionally satisfies (H2), then any function $v_{0} \in L^{2}(\Gamma)$ can be extended constantly along the characteristics of $\beta$ : there exists $v \in V_{\omega}$ such that $v_{\mid \Gamma}=v_{0}$ and $\beta \cdot \nabla v=0$.

Proof.

(i) We rather prove that $L^{2}(\Gamma)=\left\{\tilde{v}_{\mid \Gamma} \mid \tilde{v} \in \widetilde{V}_{\alpha}\right\}$, where $\alpha=\omega \circ \eta|\mathrm{D} \eta|$; see Remark 2.21. To this end, consider $\xi$ and $K$ be as in the proof of Proposition 2.20. For an arbitrary function $v_{0} \in L^{2}(\Gamma)$, we define $\tilde{v}$ by the formula

$$
\tilde{v}(y, s):=v_{0}(y) \xi(s) \text { a.e. in } W .
$$

Obviously, $\tilde{v}(y, 0)=v_{0}(y)$ and $\partial_{s} \tilde{v}(y, s)=v_{0}(y) \partial_{s} \xi(s)$, whence the Cauchy-Schwarz inequality yields:

$$
\begin{aligned}
\int_{W} \alpha \tilde{v}^{2} \mathrm{~d} s \mathrm{~d} y & =\int_{\Gamma} \int_{\zeta_{-}(y)}^{\zeta_{+}(y)} \alpha(y, s) \xi(s)^{2} v_{0}(y)^{2} \mathrm{~d} s \mathrm{~d} y<\|\alpha\|_{L^{\infty}(K)}\|\xi\|_{L^{2}(\mathbb{R})}\left\|v_{0}\right\|_{L^{2}(\Gamma)}^{2}<+\infty, \\
\int_{W} \alpha\left|\partial_{s} \tilde{v}\right|^{2} \mathrm{~d} s \mathrm{~d} y & =\int_{\Gamma}^{\zeta_{+}(y)} \int_{\zeta_{-}(y)} \alpha(y, s) v_{0}(y)^{2}\left|\partial_{s} \xi(s)\right|^{2} \mathrm{~d} s \mathrm{~d} y \leq\|\alpha\|_{L^{\infty}(K)}\left\|\partial_{s} \xi\right\|_{L^{2}(\mathbb{R})}\left\|v_{0}\right\|_{L^{2}(\Gamma)}^{2}<+\infty .
\end{aligned}
$$

Hence $\tilde{v}$ is a function in $\widetilde{V}_{\alpha}$ such that $\tilde{v}_{\mid \Gamma}=v_{0}$, which is the desired result.

(ii) If (H2) is satisfied, then for an arbitrary function $v_{0} \in L^{2}(\Gamma)$, we simply define $\tilde{v}$ by the formula:

$$
\tilde{v}(y, s):=v_{0}(y) \text { a.e. in } W .
$$

Then, clearly $\partial_{s} \tilde{v}=0$; what's more, one has $\tilde{v} \in L_{\alpha}^{2}(W)$ as follows from the following estimate:

$$
\int_{W} \alpha \tilde{v}^{2} \mathrm{~d} s \mathrm{~d} y=\int_{\Gamma} \int_{\zeta_{-}(y)}^{\zeta_{+}(y)} \alpha(y, s) v_{0}(y)^{2} \mathrm{~d} s \mathrm{~d} y \leq\left\|g_{\alpha}\right\|_{L^{\infty}(\Gamma)}\left\|v_{0}\right\|_{L^{2}(\Gamma)}^{2},
$$


where $g_{\alpha}$ is as in the statement of (H2). Hence the function $\tilde{v}$ belongs to $\widetilde{V}_{\alpha}$, and so $v:=\tilde{v} \circ \eta^{-1}$ is an element of $V_{\omega}$ which has the desired properties owing to Lemma 2.11.

We now prove a Poincaré-type inequality in the spaces $V_{\omega}$ under the additional assumptions (H2) and (H3) about the weight $\omega$.

Proposition 2.23 (Poincaré type inequality on $\left.V_{\omega}\right)$. Let $\omega \in \mathcal{C}^{0}\left(U, \mathbb{R}_{+}^{*}\right)$ be a weight satisfying the assumptions (H1)-(H3). Then there exists a constant $C>0$ (depending on $\omega$ ) such that:

$$
\forall v \in V_{\omega}, \int_{U} \omega v^{2} \mathrm{~d} x \leq C\left[\int_{\Gamma} v^{2} \mathrm{~d} y+\int_{U} \omega|\beta \cdot \nabla v|^{2} \mathrm{~d} x\right] .
$$

Proof. Introducing again $\alpha=\omega \circ \eta|\mathrm{D} \eta| \in \mathcal{C}^{0}\left(W, \mathbb{R}_{+}^{*}\right)$ and using the change of variables (2.9), we rather prove the analogous Poincaré inequality in $W$, that is:

$$
\forall \tilde{v} \in \widetilde{V}_{\alpha}, \int_{W} \alpha \tilde{v}^{2} \mathrm{~d} s \mathrm{~d} y \leq C\left[\int_{\Gamma} \tilde{v}^{2} \mathrm{~d} s+\int_{W} \alpha\left|\partial_{s} \tilde{v}\right|^{2} \mathrm{~d} s \mathrm{~d} y\right] .
$$

Furthermore, since $\mathcal{C}^{\infty}(W) \cap \widetilde{V}_{\alpha}$ is dense in $\widetilde{V}_{\alpha}$, it is enough to prove that (2.33) holds for $\tilde{v} \in \mathcal{C}^{\infty}(W) \cap \widetilde{V}_{\alpha}$, which we now do. To this end, for arbitrary $\tilde{v} \in \mathcal{C}^{\infty}(W) \cap \widetilde{V}_{\alpha}$, a use of Taylor's formula yields:

$$
\forall(y, s) \in W, \tilde{v}(y, s)=\tilde{v}(y, 0)+\int_{0}^{s} \partial_{s} \tilde{v}(y, t) \mathrm{d} t .
$$

In (2.34), the Cauchy-Schwarz inequality implies that, for $(y, s) \in W$ :

$$
\int_{0}^{s}\left|\partial_{s} \tilde{v}(y, t)\right| \mathrm{d} t \leq\left(\int_{0}^{s} \alpha^{-1}(y, t) \mathrm{d} t\right)^{1 / 2}\left(\int_{\zeta_{-}(y)}^{\zeta_{+}(y)} \alpha\left|\partial_{s} \tilde{v}\right|^{2}(y, t) \mathrm{d} t\right)^{1 / 2}
$$

Now squaring (2.34), using the Young's inequality $\left(\forall a, b \in \mathbb{R},(a+b)^{2} \leq 2 a^{2}+2 b^{2}\right)$ together with (2.35), then multiplying by $\alpha(y, s)$, we obtain:

$$
\forall(y, s) \in W, \alpha(y, s)|\tilde{v}(y, s)|^{2} \leq 2 \alpha(y, s)|\tilde{v}(y, 0)|^{2}+2 \alpha(y, s) \int_{0}^{s} \alpha^{-1}(y, t) \mathrm{d} t \int_{\zeta_{-}(y)}^{\zeta_{+}(y)} \alpha(y, t)\left|\partial_{s} \tilde{v}\right|^{2}(y, t) \mathrm{d} t .
$$

Integrating (2.36) over $W$ now results in:

$$
\begin{aligned}
\int_{W} \alpha \tilde{v}^{2} \mathrm{~d} s \mathrm{~d} y & \leq 2 \int_{\Gamma} \tilde{v}^{2}(y, 0)\left(\int_{\zeta_{-}(y)}^{\zeta_{+}(y)} \alpha(y, s) \mathrm{d} s\right) \mathrm{d} y+2 \int_{\Gamma} h_{\alpha}(y)\left(\int_{\zeta_{-}(y)}^{\zeta_{+}(y)} \alpha\left|\partial_{s} \tilde{v}\right|^{2} \mathrm{~d} s\right) \mathrm{d} y \\
& \leq 2|| g_{\alpha}\left\|_{L^{\infty}(\Gamma)} \int_{\Gamma} \tilde{v}^{2} \mathrm{~d} y+2|| h_{\alpha}\right\|_{L^{\infty}(\Gamma)} \int_{W} \alpha\left|\partial_{s} \tilde{v}\right|^{2} \mathrm{~d} s \mathrm{~d} y,
\end{aligned}
$$

where $g_{\alpha}$ and $h_{\alpha}$ are the functions featured in (H2) and (H3). This completes the proof of (2.33), and so that of Proposition 2.23.

Remark 2.24. This Poincaré type inequality is close in spirit to the "curvilinear" Poincaré inequality of Azerad $[11,12]$, who considered the weight $\omega=1$ and vector fields $\beta$ satisfying $\operatorname{div} \beta=0$. 


\subsection{Well-posedness of the variational problem (2.5)}

We are now in a position to state and prove the main result of this section.

Proposition 2.25. Let $\omega \in \mathcal{C}^{0}\left(U, \mathbb{R}_{+}^{*}\right)$ be a positive weight on $U$, satisfying the assumptions $(\mathrm{H} 1)-(\mathrm{H} 3)$. Then:

(i) For any function $f \in L_{\omega^{-1}}^{2}(U)$, there exists a unique solution $u \in V_{\omega}$ to the variational problem

$$
\text { Find } u \in V_{\omega} \text { such that } \forall v \in V_{\omega}, \int_{\Gamma} u v \mathrm{~d} s+\int_{U} \omega(\beta \cdot \nabla u)(\beta \cdot \nabla v) \mathrm{d} x=\int_{U} f v \mathrm{~d} x .
$$

(ii) The trace $u_{\mid \Gamma} \in L^{2}(\Gamma)$ of the solution $u$ to (2.37), is independent of the weight $\omega$; it is given by:

$$
u(y)=\int_{\zeta_{-}(y)}^{\zeta_{+}(y)} f \circ \eta|\mathrm{D} \eta| \mathrm{d} s \quad \text { a.e. on } \Gamma .
$$

Proof.

(i) The assumption $f \in L_{\omega^{-1}}^{2}(U)$ ensures that $v \mapsto \int_{U} f v \mathrm{~d} x$ is a continuous linear form on $V_{\omega}$ owing to the Cauchy-Schwartz inequality:

$$
\forall v \in V_{\omega},\left|\int_{U} f v \mathrm{~d} x\right| \leq\|f\|_{L_{\omega-1}^{2}(U)}\|v\|_{L_{\omega}^{2}(U)} .
$$

Moreover, the Poincaré inequality of Proposition 2.23 ensures the coercivity on $V_{\omega}$ of the bilinear form

$$
(u, v) \mapsto \int_{\Gamma} u v \mathrm{~d} y+\int_{U} \omega(\beta \cdot \nabla u)(\beta \cdot \nabla v) \mathrm{d} x .
$$

Hence, the classical Lax-Milgram theorem (see e.g. [32]) yields the existence and uniqueness of a solution $u \in V_{\omega}$ to $(2.37)$.

(ii) Since $\omega$ satisfies (H2), it follows from Proposition 2.22 (ii) that for any $v_{0} \in \mathcal{C}^{0}(\Gamma)$, there exists a function $v \in V_{\omega}$ such that $v_{\mid \Gamma}=v_{0}$ and $\beta \cdot \nabla v=0$. Taking $v$ as an admissible test function in (2.37) and using once again the change of variables (2.9) yields:

$$
\forall v_{0} \in \mathcal{C}^{0}(\Gamma), \int_{\Gamma} u v_{0} \mathrm{~d} y=\int_{U} f v \mathrm{~d} x=\int_{\Gamma} v_{0}(y)\left(\int_{\zeta_{-}(y)}^{\zeta_{+}(y)} f \circ \eta|\mathrm{D} \eta| \mathrm{d} s\right) \mathrm{d} y,
$$

whence (2.38).

Remark 2.26. Problem (2.5) or (2.37) can be solved, for an arbitrary choice of weight $\omega$ satisfying (H1)-(H3), if the right hand side satisfies $f \in L_{\omega^{-1}}^{2}(U)$. This holds true, for example when $f$ belongs to $L^{\infty}(U)$, as soon as the weight $\omega$ satisfies: $\omega^{-1} \in L^{1}(U)$. The latter property is not a consequence of (H1)-(H3) as explained in Remark 3.9.

Remark 2.27. If (H2) is not satisfied, which is the case if for example $\omega$ blows up "too fast" near some part of the boundary of $U$, functions $v \in V_{\omega}$ are expected to vanish near this part of $\partial U$ and then $V_{\omega}$ may not contain all functions which are constant along the characteristic curves of $\beta$. 


\section{NumERICAL METHODS FOR INTEGRATION ALONG NORMAL RAYS}

We now instantiate the general setting of Section 2 to the shape optimization context (2.7) outlined in the introduction. As we have mentioned, it relies heavily on the notion of signed distance function, which we briefly recall in the next Section 3.1 for the convenience of the reader. We then clarify, for comparison purposes, some of the algorithmic stages required by the direct integration of (2.6) along rays in Section 3.2 - such as the numerical computation of the principal curvatures $\kappa_{i}$ of the considered shapes, and the delicate detection of their skeleton on unstructured meshes. We discuss next in Section 3.3 the construction of suitable weights $\omega$ that allow to to solve accurately the variational problem (2.5) by means of $\mathbb{P}_{1}$ conforming finite elements. Finally, in Section 3.4, we compare on several 2-d analytical examples the numerical calculations of (2.6) by means of our variational formulation (2.5) with those produced by direct integration of this formula along rays.

\subsection{A short reminder about the signed distance function}

In this section, we consider a fixed, bounded and Lipschitz "hold-all" open domain $D \subset \mathbb{R}^{n}$, as well as a bounded Lipschitz open subdomain $\Omega \subset D$. We recall the main definitions and properties of the signed distance function that will be relevant for the shape optimization applications of Section 4, referring to [14,25,26] for further details.

Definition 3.1 (Signed distance function). For any $x \in D$, let $d(x, \partial \Omega)$ be the usual Euclidean distance from $x$ to $\partial \Omega$ :

$$
d(x, \partial \Omega):=\min _{y \in \partial \Omega}\|x-y\|
$$

where $\|\cdot\|$ denotes the Euclidean norm of $\mathbb{R}^{n}$. The signed distance function $d_{\Omega}: D \rightarrow \mathbb{R}$ to the domain $\Omega$ is defined by:

$$
\forall x \in D, \quad d_{\Omega}(x)=\left\{\begin{array}{cl}
-d(x, \partial \Omega) & \text { if } x \in \Omega, \\
0 & \text { if } x \in \partial \Omega, \\
d(x, \partial \Omega) & \text { if } x \in D \backslash \bar{\Omega}
\end{array}\right.
$$

We then recall the definitions of the projection mapping onto $\partial \Omega$ and of the skeleton of $\Omega$, which are illustrated on Figure 1A.

Definition 3.2 (Skeleton and projection).

(i) The (non empty) set of projections $\Pi_{\partial \Omega}(x)$ of a point $x \in D$ onto $\partial \Omega$ is the set of points $y \in \partial \Omega$ achieving the minimum in (3.1), that is:

$$
\Pi_{\partial \Omega}(x)=\{y \in \partial \Omega \mid\|x-y\|=d(x, \partial \Omega)\}
$$

(ii) The skeleton $\Sigma \subset D$ of $\Omega$ (relative to $D$ ) is the set of points $x \in D$ such that $\Pi_{\partial \Omega}(x)$ is not a singleton.

(iii) For $x \in D \backslash \Sigma$, the unique element in $\Pi_{\partial \Omega}(x)$ is denoted by $p_{\partial \Omega}(x)$ and is called the projection of $x$ onto $\partial \Omega$.

In the following reminders on the signed distance function $d_{\Omega}$, we suppose for simplicity that $\Omega$ is a domain of class $\mathcal{C}^{2}$ (although this assumption may not be minimal).

Proposition 3.3 (Differentiability of $d_{\Omega}$ ). The signed distance function $d_{\Omega}$ is differentiable at any point $x \in$ $D \backslash \Sigma$, and it is not differentiable on $\Sigma$. The gradient $\nabla d_{\Omega}$ is an extension of the unit normal vector $\boldsymbol{n}$ to $\partial \Omega$ pointing outward $\Omega$, in the sense that it satisfies the properties:

$$
\forall y \in \partial \Omega, \quad \nabla d_{\Omega}(y)=\boldsymbol{n}(y) \text { and } \forall x \in D \backslash \Sigma,\left\|\nabla d_{\Omega}\right\|=1
$$


Definition 3.4 (Normal rays). For $y \in \partial \Omega$, the ray emerging from $y$ is the one-dimensional segment

$$
\operatorname{ray}(y):=\left\{x \in D \backslash \bar{\Sigma}, p_{\partial \Omega}(x)=y\right\}=\left\{y+s \nabla d_{\Omega}(y) \mid s \in\left(\zeta_{-}(y), \zeta_{+}(y)\right)\right\} .
$$

where $\zeta_{-}(y)$ and $\zeta_{+}(y)$ are the maximum distances at which ray $(y)$ hits either the skeleton $\Sigma$ or the boundary $\partial D$ of the hold-all domain:

$$
\begin{aligned}
& \forall y \in \partial \Omega, \quad \zeta_{+}(y)=\sup \left\{s \geq 0 \mid\left\{y+t \nabla d_{\Omega}(y) \mid t \in[0, s)\right\} \cap(\bar{\Sigma} \cup \partial D)=\emptyset\right\}, \\
& \forall y \in \partial \Omega, \zeta_{-}(y)=\inf \left\{s \leq 0 \mid\left\{y+t \nabla d_{\Omega}(y) \mid t \in(s, 0]\right\} \cap(\bar{\Sigma} \cup \partial D)=\emptyset\right\} .
\end{aligned}
$$

The functions $\zeta_{-}$and $\zeta_{+}$are continuous on $\partial \Omega$ (see [15] or [45]).

Let us now turn to properties related to the second-order regularity of the signed distance function $d_{\Omega}$, still under the assumption that the domain $\Omega$ is at least of class $\mathcal{C}^{2}$. In all what follows, we denote for any $y \in \partial \Omega$ by $\boldsymbol{n}(y)$ the outward normal to $\Omega$. The tangential gradient $\partial_{y} \boldsymbol{n}(y)$ (with respect to the coordinates of $\partial \Omega$ ) is a symmetric tensor whose eigendecomposition

$$
\partial_{y} \boldsymbol{n}(y)=\sum_{i=1}^{n-1} \kappa_{i}(y) \boldsymbol{\tau}_{i}(y) \boldsymbol{\tau}_{i}(y)^{T}
$$

involves the principal curvatures $\left(\kappa_{i}(y)\right)_{1 \leq i \leq n-1}$ and the associated principal directions $\left(\boldsymbol{\tau}_{i}(y)\right)_{1 \leq i \leq n-1}$ of $\partial \Omega$ at $y$. Note that for any unit extension $\widetilde{\boldsymbol{n}}: \mathcal{V} \rightarrow \mathbb{R}^{n}$ of $\boldsymbol{n}$ to some tubular neighborhood $\mathcal{V} \subset D$ of $\partial \Omega$ satisfying $\widetilde{\boldsymbol{n}}(y)=\boldsymbol{n}(y)$ for any $y \in \partial \Omega$ and $\|\widetilde{n}(x)\|=1$ for any $x \in \mathcal{V}$, it holds that $\nabla \widetilde{\boldsymbol{n}}(y)=\partial_{y} \boldsymbol{n}(y)$.

The next proposition is related to the differentiability properties of the projection $p_{\partial \Omega}$, or equivalently to the second-order differentiability of $d_{\Omega}$ (see $[8,14,15,35,48]$ ).

Proposition 3.5 (Differentiability of $\left.p_{\partial \Omega}\right)$. The following properties hold true:

(1) For any point $x \in D \backslash \bar{\Sigma}$, one has:

$$
1+\kappa_{i}\left(p_{\partial \Omega}(x)\right) d_{\Omega}(x)>0, \quad i=1, \ldots, n-1 .
$$

(2) The projection $p_{\partial \Omega}$ is differentiable on $D \backslash \bar{\Sigma}$ and

$$
\forall x \in D \backslash \bar{\Sigma}, \nabla p_{\partial \Omega}(x)=\sum_{i=1}^{n-1} \frac{1}{1+\kappa_{i}\left(p_{\partial \Omega}(x)\right) d_{\Omega}(x)} \boldsymbol{\tau}_{i}\left(p_{\partial \Omega}(x)\right) \boldsymbol{\tau}_{i}\left(p_{\partial \Omega}(x)\right)^{T} .
$$

Remark 3.6. The projection $p_{\partial \Omega}$ is not differentiable on the boundary $\partial \Sigma$ of the skeleton $\Sigma$. Indeed (see Fig. 1A and [15,35]), a point $C \in D$ belongs to the closure $\bar{\Sigma}$ either because it lies on $\Sigma$, or because it is a center of curvature of $\partial \Omega$, i.e. there exists $y \in \Pi_{\partial \Omega}(C)$ and $i=1, \ldots, n-1$ such that

$$
1+d_{\Omega}(C) \kappa_{i}(y)=0 .
$$

Since $d_{\Omega}$ is Lipschitz, it follows from Rademacher's theorem (see [34], Sect. 3.1.2) that the skeleton $\Sigma$ has zero Lebesgue measure. However, if $\Omega$ is only supposed to be of class $\mathcal{C}^{2}$, the closure $\bar{\Sigma}$ may have non zero Lebesgue measure (see [48] for an example). For $\bar{\Sigma}$ to be also of null Lebesgue measure, a little more regularity is required about $\Omega$, namely, that it be at least of class $\mathcal{C}^{3}[15,48]$.

From (3.7) and (3.8), we may calculate the Hessian matrix of $d_{\Omega}$ and the Jacobian $|\mathrm{D} \eta|$. The last proposition of this section sums up the characteristics of the shape optimization setting in the perspective of the previous Section 2 . 
Proposition 3.7 (Shape optimization setting). Let $\Omega \subset D$ be a domain of class $\mathcal{C}^{2}$; then the signed distance function $d_{\Omega}$ is of class $\mathcal{C}^{2}$ on the open set $U:=D \backslash \bar{\Sigma}$. Hence $\beta:=\nabla d_{\Omega}$ is a vector field of class $\mathcal{C}^{1}$ on $U$; the associated flow map $\eta: W \rightarrow D \backslash \bar{\Sigma}$ is a diffeomorphism of class $\mathcal{C}^{1}$, whose expression reads:

$$
\forall(y, s) \in W, \eta(y, s)=y+s \nabla d_{\Omega}(y)
$$

where $W$ is the set defined by (2.3). The inverse flow mapping $\eta^{-1}: U \rightarrow W$ is given by

$$
\forall x \in U, \eta^{-1}(x)=\left(p_{\partial \Omega}(x), d_{\Omega}(x)\right) .
$$

The divergence of the vector field $\beta=\nabla d_{\Omega}$ and the Jacobian $|\mathrm{D} \eta|$ of the flow map $\eta$ are respectively given by

$$
\begin{gathered}
\forall x \in D \backslash \bar{\Sigma}, \operatorname{div}\left(\nabla d_{\Omega}\right)(x)=\Delta d_{\Omega}(x)=\sum_{i=1}^{n-1} \frac{\kappa_{i}\left(p_{\partial \Omega}(x)\right)}{1+d_{\Omega}(x) \kappa_{i}\left(p_{\partial \Omega}(x)\right)}, \\
\forall(y, s) \in W,|\mathrm{D} \eta|(y, s)=|\operatorname{det}(\nabla \eta)|(y, s)=\prod_{i=1}^{n-1}\left(1+s \kappa_{i}(y)\right) .
\end{gathered}
$$

\subsection{Computing curvatures and detecting the skeleton for direct integration along the rays}

Before going to the numerical aspects of our variational method, we clarify important practical details which are required in the implementation of the direct integration along characteristics involved in the calculation of (2.6). In Section 3.2.1, we discuss the delicate issue of detecting the triangles in the computational mesh of $D$ crossing the skeleton $\Sigma$ of $\Omega$ when traveling along the rays (note that this step is not required by our variational method). Then, we detail in Section 3.2.2 the method we used to compute the curvature $\kappa$ (there is only one curvature $\kappa:=\kappa_{1}$ in 2-d) required in the line integral formula (1.3). Note that these steps serve only for comparison purposes with our variational method. These are fairly classical numerical issues which could otherwise be addressed with more sophisticated techniques, see e.g. $[27,55]$.

For our present numerical applications, the hold-all domain $D$ is equipped with a simplicial mesh $\mathcal{T}$ featuring a discretization of the domain $\Omega$ as a submesh. However, the only information we use about $\Omega$ is an accurate approximation $d_{h}$ of the signed distance function $d_{\Omega}$ as an element of the space $V_{h}$ of Lagrange $\mathbb{P}_{1}$ finite element, where $h$ is the maximum mesh element size. Multiple methods are available to achieve the latter calculation $[57,63]$; here we rely on the numerical algorithm from the previous work [20].

\subsubsection{Detection of the skeleton $\Sigma$ of $\Omega$ and identification of normal rays}

The numerical detection of $\Sigma$ in the course of the identification of the set ray $(y)$ for some given point $y \in \partial \Omega$ is achieved by assessing the following criterion (independently of the dimension), holding at the continuous level (see [26]):

$$
\forall y \in \partial \Omega, \forall z_{0}, z_{1} \in \operatorname{ray}(y), \frac{d_{\Omega}\left(z_{1}\right)-d_{\Omega}\left(z_{0}\right)}{\left(z_{1}-z_{0}\right) \cdot \nabla d_{\Omega}(y)}=1 .
$$

In our implementation, when computing the ray emerging at some point $y \in \partial \Omega$ (which is detected by the fact that $d_{h}(y)=0$ ), we travel the triangles in the mesh $\mathcal{T}$ in the normal direction $\boldsymbol{n}=\nabla d_{h}(y)$, and we stop the calculation of the ray in the triangle $T \in \mathcal{T}$ where the entering and exiting points $z_{0}$ and $z_{1}$ satisfy:

$$
\left|\frac{d_{h}\left(z_{1}\right)-d_{h}\left(z_{0}\right)}{\left\|z_{1}-z_{0}\right\|}-\operatorname{sign}\left(d_{h}\left(z_{0}\right)\right)\right| \geq \text { tolRay }
$$

where tolRay a small tolerance (set to 0.3 in our implementation). This provides meanwhile an approximate location of the skeleton $\Sigma$, up to a tolerance of the order of the mesh size. 


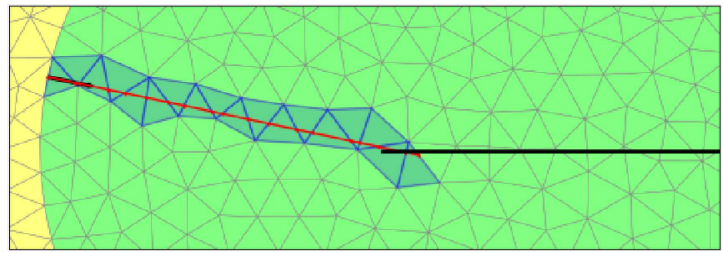

(A)

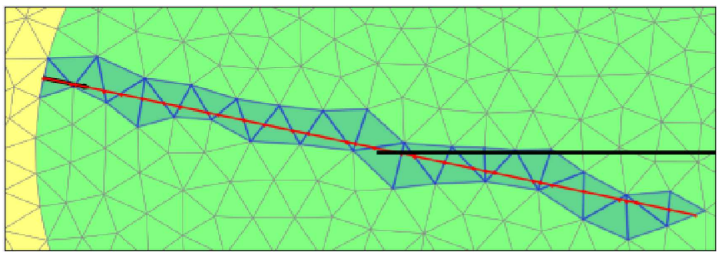

(B)

FiguRE 3. Comparison between the two criteria of Section 3.2.1 for the detection of $\Sigma$ when travelling along rays in an unstructured mesh (Skeleton displayed in the black line). (A) Criterion (3.13). (B) Criterion (3.14).

Our criterion (3.13) differs from that used in the related works $[7,50]$. In there, the authors detect $\Sigma$ by looking at changes in the monotonicity of the signed distance function $d_{h}$ along the ray, i.e. they rely on the following property of the (continuous) signed distance function $d_{\Omega}$ :

$$
\forall y \in \partial \Omega, \forall z_{0}, z_{1} \in \operatorname{ray}(y),\left(d_{\Omega}\left(z_{1}\right)-d_{\Omega}\left(z_{0}\right)\right)\left(\left(z_{1}-z_{0}\right) \cdot \nabla d_{\Omega}(y)\right) \geq 0 .
$$

Our personal experiment with the above criterion suggests that it may sometimes fail to detect the skeleton $\Sigma$ accurately, because such a change in monotonicity may simply not occur when the ray is supposed to cross $\Sigma$ in the neighborhood of center of curvatures (see Rem. 3.6). Our criterion (3.13) may also fail depending on the chosen tolerance parameter, but it offered visible improvements (Fig. 3) in our academic test-cases. Note that when integrating along the ray, the last triangle, where the skeleton is hit, is included in the integration.

\subsubsection{Estimating the curvature $\kappa$ of a 2-d subdomain based on its signed distance function}

In this part, we detail our method for the numerical approximation, in 2-d, of the principal curvature $\kappa$ (there is only one in 2-d) from the knowledge of a $\mathbb{P}^{1}$ discretization $d_{h}$ of the signed distance function $d_{\Omega}$ at the nodes of the mesh $\mathcal{T}$. We essentially rely on the fact that in 2 -d, $\kappa$ is given by the trace of $\Delta d_{\Omega}$ on the boundary $\partial \Omega$ (in view of (3.11). In 3-d, the estimation of $\Delta d_{\Omega}$ would not be sufficient to evaluate the values of both principal curvatures $\kappa_{1}$ and $\kappa_{2}$ : these could e.g. be computed from the eigenvalues of the Hessian matrix $\nabla^{2} d_{\Omega}$.

Our first step towards estimating $\Delta d_{\Omega}$ consists in calculating a $\mathbb{P}_{1}$ interpolation $g_{h} \in V_{h} \times V_{h}$ of the piecewise constant gradient $\nabla d_{h}$ by solving the following variational problem:

$$
\forall \psi_{h} \in V_{h} \times V_{h}, \int_{D} g_{h} \cdot \psi_{h} \mathrm{~d} x=\int_{D} \nabla d_{h} \cdot \psi_{h} \mathrm{~d} x .
$$

The approximation of the divergence $\operatorname{div}\left(\nabla d_{\Omega}\right)$ is then calculated as the (piecewise constant) divergence of the reconstructed field $g_{h}$. Unfortunately, this procedure generally produces a very noisy approximation characterized by a lot of spurious oscillations when the mesh resolution increases (see Fig. 4). In order to overcome this difficulty, we calculate a regularization $\kappa_{h}$ of this noisy estimation with a Laplace kernel, namely we solve:

$$
\text { Find } \kappa_{h} \in V_{h} \text { such that } \forall \psi_{h} \in V_{h}, \int_{D}\left(\gamma_{h}^{2} \nabla \kappa_{h} \cdot \nabla \psi_{h}+\kappa_{h} \psi_{h}\right) \mathrm{d} x=\int_{D} \operatorname{div}\left(g_{h}\right) \psi_{h} \mathrm{~d} x \text {, }
$$

where $\gamma_{h}>0$ is a regularization length scale (equal to $3 h_{\max }$ where $h_{\max }$ is the maximum edge length in the mesh). This procedure yields satisfying results in practice (even with shapes $\Omega$ characterized by discontinuous curvatures, up to some over smoothing near the discontinuities), although we do not have a proof of convergence of the approximation $\kappa_{h}$ towards the exact function $\Delta d_{\Omega}$. 


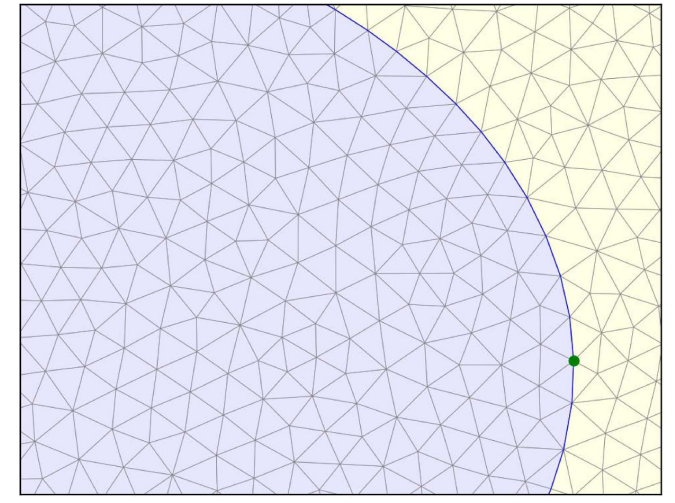

(A)

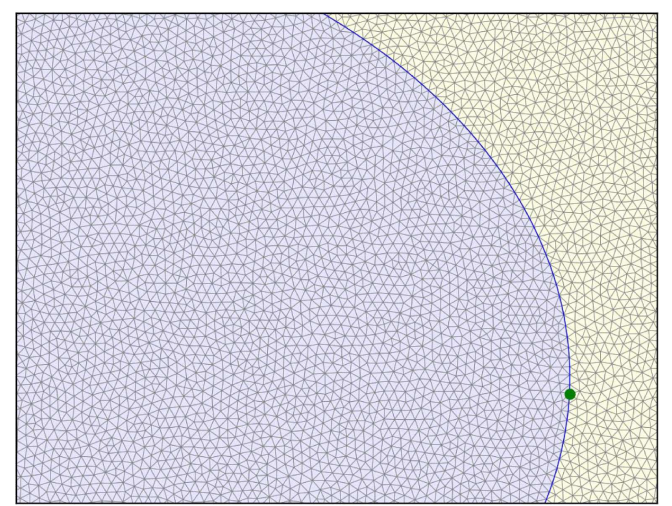

(C)

Figure 4. Estimation of $\Delta d_{\Omega}$ on the mesh $\mathcal{T}$ for the shape $\Omega$ in (3.17) and for two different mesh resolutions. The $x$ coordinate on the right-hand graphs represents the arc length coordinate on $\partial \Omega$ when the starting point is the green reference point. Estimates of the mean curvature of $\partial \Omega, \operatorname{div}\left(g_{h}\right)$ and $\kappa_{h}$ (see (3.15) and (3.16) for the definitions) are compared to the analytical value $\kappa(y)$. (A) Zoom on a mesh discretization of an ellipse $\Omega \subset D$ with a maximum edge length $h_{\max }=0.08$. (B) Estimated values of $\Delta d_{\Omega}$ on $\partial \Omega$. (C) Zoom on a finer mesh discretization of $\Omega \subset D$ with a maximum edge length $h_{\max }=0.02$. (D) Estimated values of $\Delta d_{\Omega}$ on $\partial \Omega$.

Let us illustrate our method by considering the example of an ellipse $\Omega$ inside a square-shaped hold-all domain $D$ : let $D$ and $\Omega$ be defined by

$$
D=\left\{\left(x_{1}, x_{2}\right) \in \mathbb{R}^{2}|| x_{1} \mid<2 \text { and }\left|x_{2}\right|<2\right\}, \Omega=\left\{\left(x_{1}, x_{2}\right) \in D \mid \frac{x_{1}^{2}}{a^{2}}+\frac{x_{2}^{2}}{b^{2}}<1\right\}
$$

where $a=1.5$ and $b=1$. The skeleton of $\Omega$ is explicitly known in this case: $\Sigma=\left\{\left(x_{1}, 0\right)|| x_{1} \mid<a-b^{2} / a\right\}$ and the curvature $\kappa$ of $\partial \Omega$ at a point $y=\left(y_{1}, y_{2}\right)$ is given by $\kappa(y)=a b / \gamma^{3}$ with $\gamma=\sqrt{\frac{b^{2}}{a^{2}} y_{1}^{2}+\frac{a^{2}}{b^{2}} y_{2}^{2}}$. The difference between the exact curvature $\kappa(y)$ of $\partial \Omega$ and its reconstruction $\kappa_{h}$ (at the boundary nodes discretizing $\partial \Omega$ ) using both procedures (3.15) and (3.16) is represented in Figure 4. 


\subsection{Admissible numerical weights built upon the signed distance function}

In this section, we discuss the numerical resolution of the variational problem (2.5) in the shape optimization context (2.7) and (2.8) (see also Prop. 3.7). The numerical setting is the same as that of the previous Section 3.2: the hold-all domain $D$ is equipped with a simplicial mesh $\mathcal{T}$ (i.e. composed of triangles in 2-d, or tetrahedra in 3 -d, although our method would work with other kinds of meshes) featuring a discretization of the domain $\Omega$ as a submesh, and we rely on the Lagrange $\mathbb{P}_{1}$ finite element method for the discretization of (2.5) on account of its robustness and ease of implementation. In other terms, the approximation $u_{h}$ to the solution $u \in V_{\omega}$ of (2.5) is sought in the space $V_{h}$ of continuous piecewise linear functions on each simplex of $\mathcal{T}$.

We start in Section 3.3.1 by introducing some motivations for the use in (2.5) of weights $\omega$ vanishing on the skeleton, and we provide (in Cor. 3.8 below) a formula for analytical and admissible weights satisfying approximately this property. We then discuss the issue of computing numerically these weights in Section 3.3.2. Finally, we perform a few numerical experiments in Section 3.3.3 where we show that weights vanishing on the skeleton make $\mathbb{P}_{1}$ finite elements able to capture discontinuous test functions across the skeleton, which allows to confirm numerically the latter motivations.

\subsubsection{Motivations for weights vanishing on the skeleton}

As already mentioned, our final target is to calculate the trace (2.6) on $\Gamma=\partial \Omega$ of $u$. In the continuous setting of Section 2, this trace does not depend on the choice of the weight $\omega$ as long as it fulfills (H1)-(H3), so that in principle, any such weight could be used. However, when $U$ is a cracked domain (typically, the skeleton $\Sigma$ is a crack in the working domain, i.e., $U=D \backslash \bar{\Sigma}$ ) and the crack is not explicitly discretized in the mesh $\mathcal{T}$, then the most simple choice $\omega=1$ (which is an admissible weight on account of Cor. 3.8 below) might not work well in practice. Indeed and as we shall illustrate below in Section 3.3.3, test functions of (2.37) which belong to $V_{\omega}$ with $\omega=1$ are in general discontinuous across $\Sigma$ and not well captured by $\mathbb{P}_{1}$ finite elements. In many shape optimization applications, discretizing the skeleton $\Sigma$ of the current domain $\Omega$ at every iteration of the optimization process or resorting to discontinuous finite elements (in order to be able to set $\omega=1$ ) is very inconvenient, for instance if working with fixed structured meshes as it is performed in many applications built on the level set method $[3,61]$.

Therefore, we shall be interested in determining weights $\omega$ adapted to our commitment to use the space $V_{h}$ of Lagrange $\mathbb{P}_{1}$ finite elements for the resolution of (2.5) without the need for an accurate discretization of the skeleton $\Sigma$ (alternative approaches could be to use discontinuous finite elements close to the skeleton, or to have a zero weight on the degrees of freedom corresponding to modes close to the skeleton and to remove the null space in the corresponding linear system, but they seemed to be more complicated to implement, at least to us). The weight $\omega$ should be chosen in such a way that arbitrary functions $v \in V_{\omega}$ are well approximated (in the norm $\|\cdot\|_{V_{\omega}}$ ) by functions $v_{h} \in V_{h}$, as is reflected by the classical Céa's lemma (see e.g. [32]):

$$
\left\|u-u_{h}\right\|_{V_{\omega}} \leq C \inf _{v_{h} \in V_{h}}\left\|u-v_{h}\right\|_{V_{\omega}}
$$

for a constant $C>0$ (which possibly depends on $\omega$ ). The space $V_{h}$ of Lagrange $\mathbb{P}_{1}$ elements is a conformal finite element space in the sense that the inclusion $V_{h} \subset V_{\omega}$ always holds (because functions of $V_{h}$ are smoother than those of $V_{\omega}$ ), however $V_{h}$ may be "too small" to guarantee a correct approximation of discontinuous solutions $u \in V_{\omega}$ in the sense of (3.18). Heuristically, and without looking for a very precise statement, these considerations call for the choice of a weight $\omega$ almost vanishing on $\bar{\Sigma}$, so that the approximation error $\left\|u-v_{h}\right\|_{V_{\omega}}$ in (3.18) attributes a lesser weight to a neighborhood of $\bar{\Sigma}$ where $u$ is expected to be discontinuous while the functions $v_{h} \in V_{h}$ are continuous.

We now provide explicit candidates for weights $\omega$ which fulfill the conditions (H1)-(H3) while taking small values near the skeleton, that we are going to use in our practical implementations.

Lemma 3.8. For any real numbers $q \geq 0, r \geq 0$, the weight

$$
\omega=\frac{1}{1+\left|d_{\Omega}\right|^{q}\left|\Delta d_{\Omega}\right|^{r}}
$$


satisfies the conditions (H1)-(H3) (this includes in particular the constant weight $\omega=1 / 2$ for $q=r=0$ ).

Proof. At first, it follows readily from the definition that $\omega$ belongs to $\mathcal{C}^{0}\left(U, \mathbb{R}_{+}^{*}\right)$, and is uniformly bounded on $U$, so that (H1) and (H3) are trivially satisfied. We then define $\kappa_{-}(y)$ and $\kappa_{+}(y)$ by:

$$
\kappa_{-}(y)=\min \left(0, \min _{i} \kappa_{i}(y)\right), \kappa_{+}(y)=\max \left(0, \max _{i} \kappa_{i}(y)\right),
$$

as well as the corresponding multiplicities $m_{-}(y)$ and $m_{+}(y)$ :

$$
m_{ \pm}(y)=\operatorname{Card}\left(\left\{i \in\{1, \ldots, n-1\} \mid \kappa_{i}(y)=\kappa_{ \pm}(y)\right\}\right) .
$$

Using formula (3.11) for $\Delta d_{\Omega}$, the weight $\alpha=\omega \circ \eta|\mathrm{D} \eta|$ is decomposed as

$$
\begin{aligned}
\alpha(y, s)=\frac{\prod_{i=1}^{n-1}\left(1+\kappa_{i}(y) s\right)}{1+|s|^{q}\left|\sum_{i} \frac{\kappa_{i}(y)}{1+\kappa_{i}(y) s}\right|^{r}} & =\frac{\prod_{i=1}^{n-1}\left(1+\kappa_{i}(y) s\right)^{r+1}}{\prod_{i=1}^{n-1}\left(1+\kappa_{i}(y) s\right)^{r}+|s|^{q}\left|\sum_{i} \kappa_{i}(y) \prod_{j \neq i}\left(1+\kappa_{j}(y) s\right)\right|^{r}} \\
& =f(y, s) g(y, s)
\end{aligned}
$$

where $f$ and $g$ are the following functions:

$$
\begin{gathered}
f(y, s)=\left(1+s \kappa_{-}(y)\right)^{m_{-}(y)(r+1)} \mathbf{1}_{s \geq 0}(s)+\left(1+s \kappa_{+}(y)\right)^{m_{+}(y)(r+1)} \mathbf{1}_{s<0}(s), \\
g(y, s)=\frac{\prod_{i, \kappa_{i} \neq \kappa_{-}}\left(1+\kappa_{i}(y) s\right)^{r+1} \mathbf{1}_{s \geq 0}(s)+\prod_{i, \kappa_{i} \neq \kappa_{+}}\left(1+\kappa_{i}(y) s\right)^{r+1} \mathbf{1}_{s<0}(s)}{\prod_{i=1}^{n-1}\left(1+\kappa_{i}(y) s\right)^{r}+|s|^{q}\left|\sum_{i} \kappa_{i}(y) \prod_{j \neq i}\left(1+\kappa_{j}(y) s\right)\right|^{r}} .
\end{gathered}
$$

Then, $f$ satisfies clearly the monotonicity condition (ii) in the statement of Lemma 2.2 and $g$ is a continuous function on each of the domains $W_{-}=\{(y, s) \in W \mid s \leq 0\}$ and $W_{+}=\{(y, s) \in W \mid s \geq 0\}$, that does not vanish on the compact sets $\overline{W_{-}}$and $\overline{W_{+}}$. The assumptions of Lemma 2.2 are therefore fulfilled, so that $\omega$ satisfies (H3). This terminates the proof.

Remark 3.9. - When it comes to solving (2.5) by relying on Proposition 2.25 with some data $f \in L^{\infty}(U)$, it is useful to observe that $f$ belongs to $L_{\omega^{-1}}^{2}(U)$ as soon as the weight $\omega$ satisfies $\omega^{-1} \in L^{1}(U)$ (see Rem. 2.26), which is the case if it is of the form (3.19) with $r<2$. In the following numerical experiments, we shall see however that using values for $r$ which are larger than 2 still provides good results in practice: in general, taking higher values of $q$ and $r$ yields a faster decay of $\omega$ near the skeleton.

- Taking $r>0$ ensures that the weight (3.19) will vanish at points $x \in \bar{\Sigma}$ that are centers of curvatures (for which $\Delta d_{\Omega}$ blows up). Taking $q>0$ allows to make sure that $\omega=1 / 2$ on $\partial \Omega$ whatever the value of $r$, and to accentuate the decay of $\omega$ near the skeleton. Not that in general, $\omega$ will not vanish on points $x \in \bar{\Sigma}$ that are not centers of curvatures. However, it still takes very small values on $\bar{\Sigma}$ and it is convenient to use in the implementation. There is no unicity of weights appropriate for the numerical computation and variants can easily be imagined.

- For the most general setting where $U$ is an arbitrary open set, the methodology of this section extends naturally by considering weights $\omega$ which vanish on the cracked parts of $\partial U$ that are not explicitly meshed.

\subsubsection{Numerical computations of the weight based on the Laplacian of the signed distance function}

For our numerical applications below, we shall use the weights of Corollary 3.8 in the definition of our variational formulation (2.5). This requires the computation of the Laplacian $\Delta d_{\Omega}$ on the triangulated mesh $\mathcal{T}$ based on the $\mathbb{P}^{1}$ estimation of the signed distance function $d_{\Omega}$. For this purpose, we use the same regularization method outlined in Section 3.2.2 for the computation of the numerical curvature.

Importantly, from Proposition 2.25, the variational formulation (2.5) is rather insensitive to the choice of the weight $\omega$, and as a result the estimation of $\Delta d_{\Omega}$ does not need to be very accurate as long as it takes large 
values near the skeleton (as we shall illustrate below in Sect. 3.3.3). In contrast, the estimation of the principal curvatures $\kappa_{i}$ for the direct method would need to be accurate. From a numerical standpoint, the formula (3.19) featuring $\Delta d_{\Omega}$ at the denominator is convenient to obtain numerically vanishing weights near the skeleton (even if (3.19) truely vanish for $r>0$ at centers of curvatures). Indeed, $\nabla d_{\Omega}$ is discontinuous across the skeleton, which should reflect in high numerical values of $\Delta d_{\Omega}$ when computing numerically the divergence $\operatorname{div}\left(\nabla d_{\Omega}\right)$ with the method of Section 3.2.2.

\subsubsection{Assessing the choice of the weight $\omega$ when using a $\mathbb{P}_{1}$ discretization: generating numerical test functions constant along rays}

In this section, we perform several numerical experiments about the influence of the choice of the weight function $\omega$ in the resolution of the variational problem (2.5) using the Lagrange $\mathbb{P}_{1}$ finite element method. With this perspective in mind, and in the 2-d numerical setting described in Sections 3.3 and 3.2, we consider the issue of generating numerical functions $v \in V_{\omega}$ which are constant along the normal rays to the shape $\Omega$. Namely, we solve the boundary-value problem

$$
\left\{\begin{aligned}
\nabla d_{\Omega} \cdot \nabla v & =0 \quad \text { in } D \backslash \bar{\Sigma} \\
v & =v_{0} \quad \text { on } \partial \Omega
\end{aligned}\right.
$$

for given data $v_{0} \in L^{2}(\partial \Omega)$.

Using the variational setting of Section 2, we show that it is possible to obtain the solution $v$ to (3.24) by solving a variational problem of the same nature of (2.5).

Proposition 3.10. Let $\omega \in \mathcal{C}^{0}\left(D \backslash \bar{\Sigma}, \mathbb{R}_{+}^{*}\right)$ be a weight satisfying (H1)-(H3). There exists a unique solution $v \in V_{\omega}$ to the following variational problem:

$$
\text { Find } v \in V_{\omega} \text { such that } \forall w \in V_{\omega}, \int_{\partial \Omega} v w \mathrm{~d} s+\int_{D \backslash \bar{\Sigma}} \omega\left(\nabla d_{\Omega} \cdot \nabla v\right)\left(\nabla d_{\Omega} \cdot \nabla w\right) \mathrm{d} x=\int_{\partial \Omega} v_{0} w \mathrm{~d} s .
$$

The solution $v$ is independent of the choice of $\omega$ as long as $(\mathrm{H} 1)-(\mathrm{H} 3)$ are satisfied, and it is given by the formula:

$$
v(x)=v_{0}\left(p_{\partial \Omega}(x)\right), \text { a.e. } x \in D \backslash \bar{\Sigma} .
$$

Proof. To see that (3.24) and (3.25) are equivalent, it is sufficient to take $v \in V_{\omega}$ constant along rays in (3.25) as in the proof of Proposition 2.25, which yields $v=v_{0}$ on $\partial \Omega$, and then $\nabla d_{\Omega} \cdot \nabla v=0$.

The formulation (3.25) is to be compared to the so-called Galerkin Least Square formulation and SUPG methods for advection-reaction problems [32], with the difference that usual assumptions of uniformly bounded divergence (which do not hold in our applications) are replaced with the regularity assumptions of Section 2.1.

Remark 3.11. The problem of building constant functions along normal rays of the form (3.24) may be solved on unstructured meshes owing to variants of the fast marching algorithm; see e.g. [19].

We now verify that a good approximation of the solution $v$ to (3.24) (or more precisely (3.25)), which is in particular discontinuous across the skeleton $\Sigma$ of $\Omega$, can be obtained either by truncating manually the skeleton from the computational mesh $\mathcal{T}$ and taking the weight $\omega=1$, or by selecting a weight $\omega$ taking "small" values near $\Sigma$. For the latter experiment, we use the weight $\omega=1 /\left(1+\left|d_{\Omega}\right|^{3.5}\left|\Delta d_{\Omega}\right|^{2}\right.$ ) (see also Rem. 3.9 about the "small" values of $\omega$ near $\Sigma$ ). Of course, removing the skeleton from the mesh is not a straightforward task in full generality but it is performed here for the sake of comparison.

Let us consider again the ellipse example of (3.17). We consider two different computational meshes $\mathcal{T}$ and $\mathcal{T}^{\prime}$. The former is a triangular mesh of $D$, and the latter $\mathcal{T}^{\prime}$ is a triangular mesh of $D \backslash \bar{\Sigma}$ (i.e. the skeleton $\bar{\Sigma}$ has been manually removed). In both $\mathcal{T}$ and $\mathcal{T}^{\prime}$, the considered shape $\Omega$ is explicitly discretized as a submesh; see Figure 5 for an illustration. 


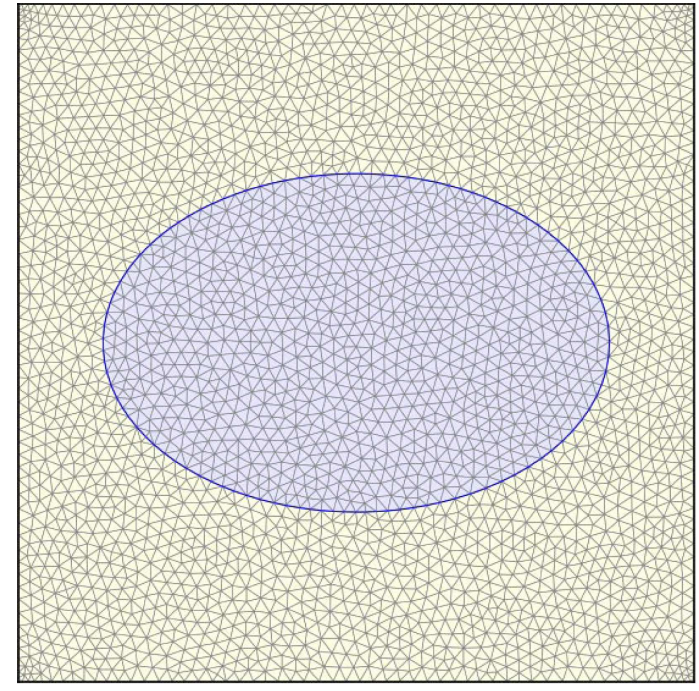

(A)

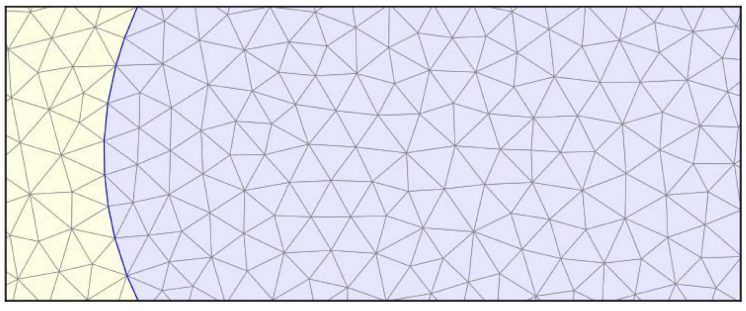

(в)

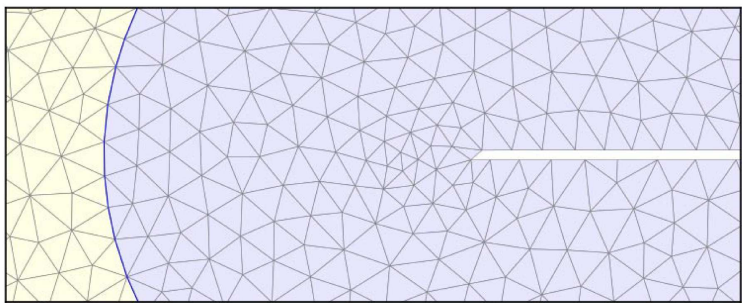

(c)

Figure 5. The two meshes $\mathcal{T}$ and $\mathcal{T}^{\prime}$ used for the test-case of Section 3.3.3. (A) Mesh $\mathcal{T}$ of the hold-all domain $D$ with $\Omega \subset D$ (in blue) discretized as a submesh. (B) Zoom on the mesh $\mathcal{T}$.

(c) Zoom on the mesh $\mathcal{T}^{\prime}$ (skeleton $\Sigma$ truncated). (Color online).

The variational problem (3.25) is numerically solved for a boundary datum $v_{0} \in L^{2}(\partial \Omega)$ given by (see Fig. 6D):

$$
\forall\left(y_{1}, y_{2}\right) \in \partial \Omega, v_{0}\left(y_{1}, y_{2}\right)=\cos \left(3 y_{1}\right)^{2}+20 y_{2},
$$

and the computed finite element solution is plotted on Figure 6 in the following three situations.

- The mesh $\mathcal{T}^{\prime}$ is used, in which $D$ and $\Omega \subset D$ are meshed explicitly, and where a thin layer around $\bar{\Sigma}$ has been manually removed (see Fig. 5C). The solution $v$ to (3.25) is computed using the constant weight $\omega=1$ and the result is displayed on Figure $6 \mathrm{~A}$. As expected, the fact that $\bar{\Sigma}$ is absent from $\mathcal{T}^{\prime}$ allows the numerical solution $v$ to have very different values on either sides of $\bar{\Sigma}$.

- The mesh $\mathcal{T}$ of $D$ (where $\bar{\Sigma}$ has not been removed) is used (see Fig. 5B), and $v$ is computed with the constant weight $\omega=1$; the result is represented on Figure 6B. The formulation (3.25) proves numerically stable with the choice $\omega=1$, but it tends to over smoothen the sharp discontinuities of $v$ near the skeleton $\Sigma$, which results in a loss of accuracy for the extension problem (3.24).

- The mesh $\mathcal{T}$ of $D$ is used again, but the solution $v$ to (3.25) is now computed by using the weight $\omega=$ $2 /\left(1+\left|d_{\Omega}\right|^{3.5}\left|\Delta d_{\Omega}\right|^{2}\right)$; the result is represented on Figure 6C. The obtained numerical solution is much closer to the expected result (3.26): the values of the solution function $v$ look constant along the normal rays up to a small neighborhood of the skeleton (of the size of the mesh element size), where sharp variations are observed, as expected. The numerical procedure in this case seems therefore to achieve the same order of accuracy than in the experiment using the truncated mesh $\mathcal{T}^{\prime}$.

\subsection{Numerical comparisons between the variational method and direct integration along rays}

We now investigate the numerical evaluation of the function $u \in L^{2}(\partial \Omega)$ in (2.6) for several 2-d academic configurations as far as $D, \Omega$ and the function $f$ are concerned. In particular, we compare the evaluation of $u$ obtained by direct integration along rays (i.e. implementing directly the formula (2.6)) to that obtained by 


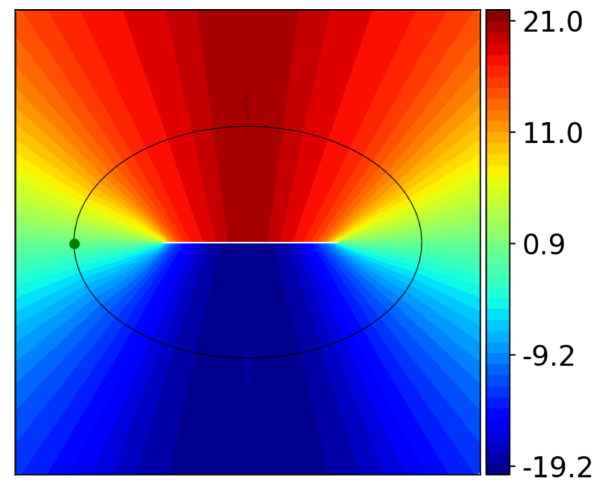

(A)

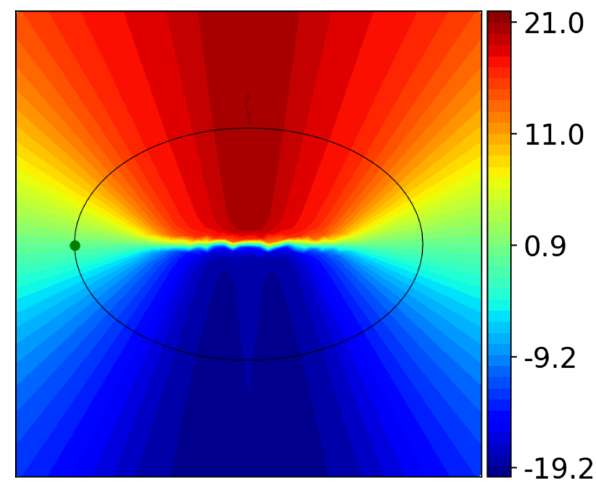

(c)

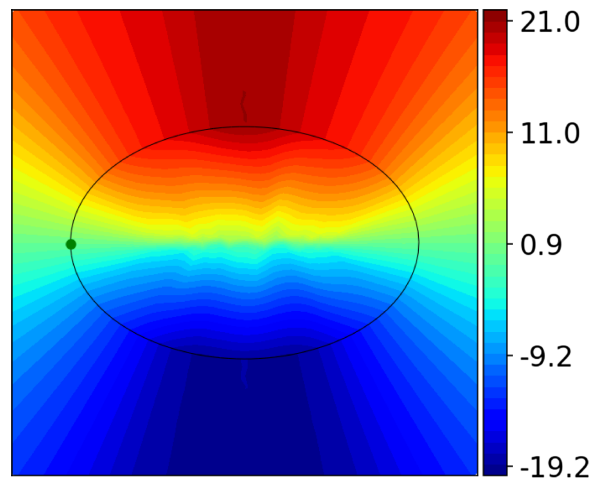

(B)

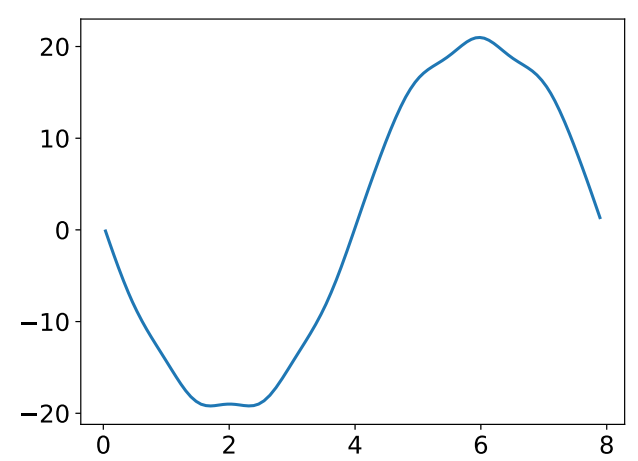

(D)

FiguRE 6. Numerical resolution of the problem (3.24) using the variational problem (3.25) for various weights, with or without removing the skeleton $\Sigma$ from the computational mesh. (A) Mesh $\mathcal{T}^{\prime}, \omega=1$. (B) Mesh $\mathcal{T}, \omega=1$. (C) Mesh $\mathcal{T}, \omega=2 /\left(1+\left|d_{\Omega}\right|^{3.5}\left|\Delta d_{\Omega}\right|^{2}\right)$. (D) Input function $v_{0}\left(y_{1}, y_{2}\right)=\cos \left(3 y_{1}\right)^{2}+20 y_{2}$ for the problem (3.25). The horizontal coordinate is the arc length with the green point as reference point. (Color online).

solving the variational formulation (2.5) on meshes $\mathcal{T}$ (resp. $\mathcal{T}^{\prime}$ ) of $D$ in which $\Omega$ is explicitly discretized and the skeleton $\Sigma$ of $\Omega$ is not removed (resp. is removed).

\subsubsection{A domain with trivial skeleton: the case of a circle}

We first consider the case where $\Omega$ is a disk enclosed in a larger disk $D$ :

$$
D=\left\{\left(x_{1}, x_{2}\right) \in \mathbb{R}^{2} \mid x_{1}^{2}+x_{2}^{2}<4\right\}, \text { and } \Omega=\left\{\left(x_{1}, x_{2}\right) \in \mathbb{R}^{2} \mid x_{1}^{2}+x_{2}^{2}<1\right\}
$$

see Figure 7. In this case, the skeleton $\Sigma$ is reduced to the point 0 . The considered function $f$ is:

$$
\forall x=\left(x_{1}, x_{2}\right) \in D, \quad f\left(x_{1}, x_{2}\right)=x_{2},
$$

which belongs to the finite element space $V_{h}$, and is therefore amenable to an exact integration when the travel procedure along rays of Section 3.2.1 is used.

In this situation, the sought function $u$, given by (2.6), is known analytically; a calculation in polar coordinates indeed yields:

$$
\forall\left(y_{1}, y_{2}\right) \in \partial \Omega, u\left(y_{1}, y_{2}\right)=\frac{8}{3} y_{2} .
$$




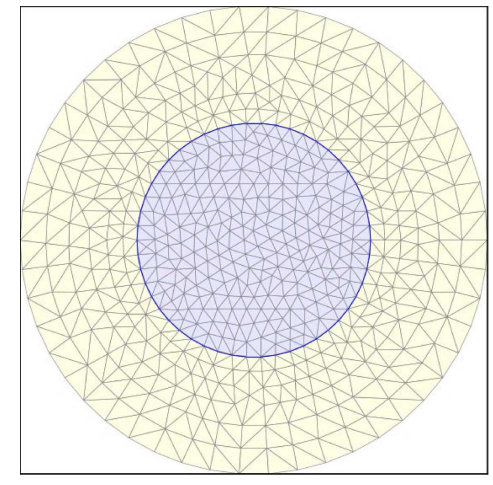

(A)

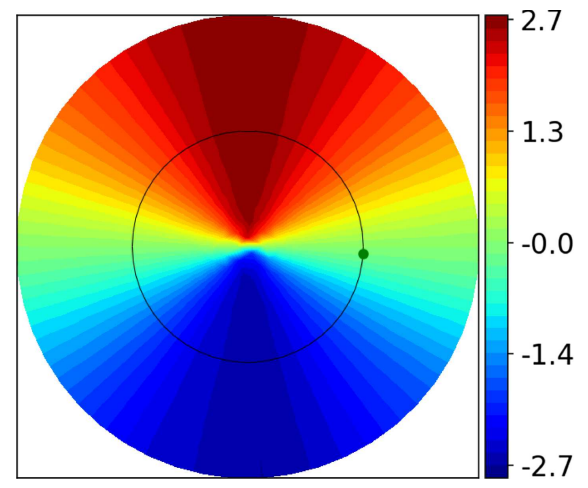

(B)

Figure 7. Setting of Section 3.4.1. (A) The mesh $\mathcal{T}$ for $D$ (part corresponding to $\Omega$ in blue). (B) The numerical solution $u$ extended constantly along rays. The green point provides the 0 reference for the horizontal axes of Figure 8. (Color online).

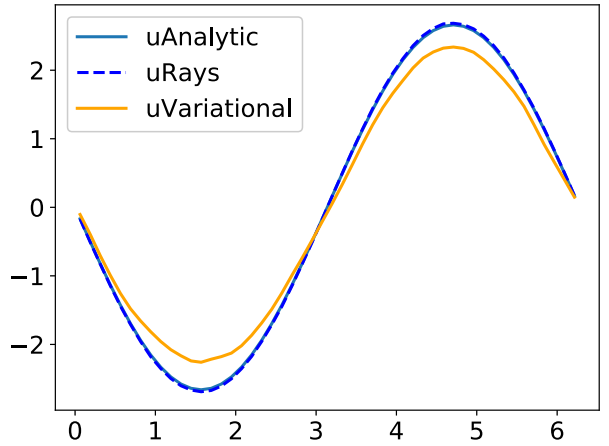

(A)

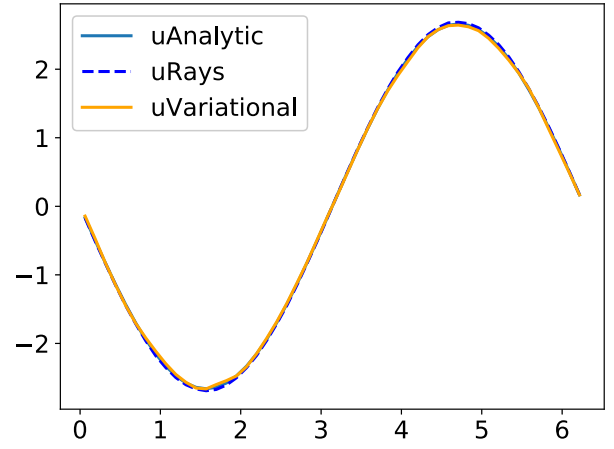

(B)

FIgURE 8. Comparison between direct integration along rays, analytical formula (1.3), and finite element solution of (2.5) for two choices of weights $\omega$ for the example of Section 3.4.1. In the legends of this figure and all those to follow, uAnalytic, uRays and uVariational refer respectively to the analytical value of $u$, its numerical estimation using (2.38), and the trace of the variational solution of (2.5). (А) $\omega=1$. (В) $\omega=2 /\left(1+\left|d_{\Omega}\right|^{3.5}\left|\Delta d_{\Omega}\right|^{3.5}\right)$. (Color online).

Comparisons are displayed on Figure 8 between the version of $u$ obtained after direct integration along rays, and the numerical solutions of (2.5) obtained for various choices of weight functions $\omega$ on the mesh $\mathcal{T}$ of $D$ (where $\Sigma$ has not been removed). We observe a good match between the variational and the direct method. As expected, the solutions computed thanks to our variational method are less accurate when the constant weight $\omega=1$ is chosen. A significant increase in accuracy is achieved by selecting a weight $\omega$ vanishing at the center of the circle, i.e. $\omega=1 /\left(1+\left|d_{\Omega}\right|^{3.5}\left|\Delta d_{\Omega}\right|^{3.5}\right)$. Note that this weight does not fulfill the condition $\omega \in L_{\omega^{-1}}^{1}(U)$ (see Rem. 2.27), but works well in numerical practice.

\subsection{2. $A \mathcal{C}^{2}$ domain with non trivial skeleton}

We take on the example (3.17) where $\Omega$ is an ellipse inside a square-shaped hold-all domain $D$, reusing both meshes $\mathcal{T}$ and $\mathcal{T}^{\prime}$ depicted on Figure 5. To evaluate the influence of the skeleton on our numerical method, we 


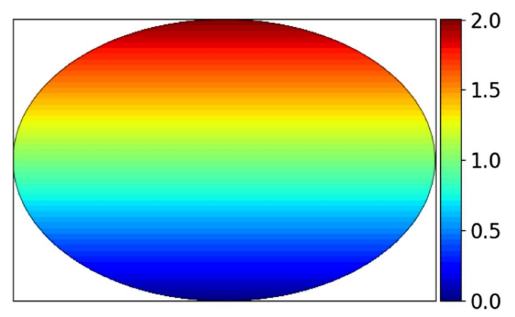

Figure 9. The function $f$ for the identification problem (2.6) for the test case of Section 3.4.2. (Color online).

compute the quantity (2.5) for a function $f$ which is supported in $\Omega$ only (see Fig. 9):

$$
f\left(x_{1}, x_{2}\right)=\left(1+x_{2}\right) \mathbf{1}_{\Omega}\left(x_{1}, x_{2}\right) .
$$

The presence of the constant 1 in (3.29) avoids the "simplification" of having $f$ vanishing on $\Sigma$. After an explicit calculation based on (2.6), the exact solution is given by:

$$
\forall y=\left(y_{1}, y_{2}\right) \in \partial \Omega, u(y)=\left(1+y_{2}\right) \zeta_{-}-\frac{\zeta_{-}^{2}}{2}\left(\frac{y_{2}}{\zeta_{-}}+\kappa\left(1+y_{2}\right)\right)+\frac{y_{2}}{3} \kappa \zeta_{-}^{2},
$$

with $\kappa=\frac{a b}{\gamma^{3}}, \zeta_{-}=\frac{\gamma b}{a}, \gamma=\sqrt{\frac{b^{2}}{a^{2}} y_{1}^{2}+\frac{a^{2}}{b^{2}} y_{2}^{2}}$.

The numerical trace $u$ (which is extended along rays using (3.25) to ease the visualization) obtained by solving the variational formulation (2.5) on $\mathcal{T}$ or $\mathcal{T}^{\prime}$, and for various choices of weights, is represented on Figure 10. On Figure 11, we compare for each of these strategies the boundary values of the numerical trace $u$ to that computed with (1.3) by direct integration along rays, and to the analytical expression (3.30).

We note that the solutions obtained by integrating along rays are generally characterized by small spurious oscillations. We explain this error by the fact that our criterion (3.13) detects the skeleton up to an error proportional to the mesh size (the actual location of the skeleton is not estimated within the last travelled triangle; see Fig. 12). Such a procedure could be improved by using more accurate skeleton detection methods; see for instance the works $[9,10]$ in the field of computer graphics. We verify that the amplitude of these oscillations decreases with the size of the mesh, as shown on Figure 11D.

\subsection{3. $A \mathcal{C}^{1}$ domain, with discontinuous curvature}

We now consider the case where $\Omega$ is a stadium, i.e. the reunion of a rectangle and two half-disks. Define (see Fig. 13)

$$
D=\left\{\left(x_{1}, x_{2}\right) \in \mathbb{R}^{2}|| x_{1} \mid \leq 2 \text { and }\left|x_{2}\right|<2\right\}
$$

and

$$
\Omega=\left\{\left(x_{1}, x_{2}\right) \in D \mid\left(\left|x_{1}\right|<0.5 \text { and }\left|x_{2}\right|<0.5\right) \text { or }\left(x_{1}-0.5\right)^{2}+x_{2}^{2}<0.25 \text { or }\left(x_{1}+0.5\right)^{2}+x_{2}^{2}<0.25\right\} .
$$

The domain $\Omega$ is not of class $\mathcal{C}^{2}$, and the curvature $\kappa$ of the boundary $\partial \Omega$ is discontinuous at the points where $x_{1}= \pm 0.5$. Hence, this example does not fall into the admissible setting of Proposition 3.7, and there is, in principle, no guarantee that our variational method based on (2.5) should still work.

In this example, the skeleton $\Sigma$ of $\Omega$ is explicitly given by $\Sigma=\left\{\left(x_{1}, 0\right)|| x_{1} \mid \leq 0.5\right\}$. We calculate the function $u$ in (2.6) associated to the datum function $f$ defined by (3.29); the analytical solution $u$ for this problem is given by

$$
u\left(y_{1}, y_{2}\right)=\left\{\begin{array}{lll}
\frac{1}{2}+\frac{1}{8} \operatorname{sign}\left(y_{1}\right) & \text { if } & \left|y_{1}\right|<0.5 \\
\frac{1}{4}+\frac{y_{2}}{6} & \text { if } & \left|y_{1}\right|>0.5
\end{array}\right.
$$




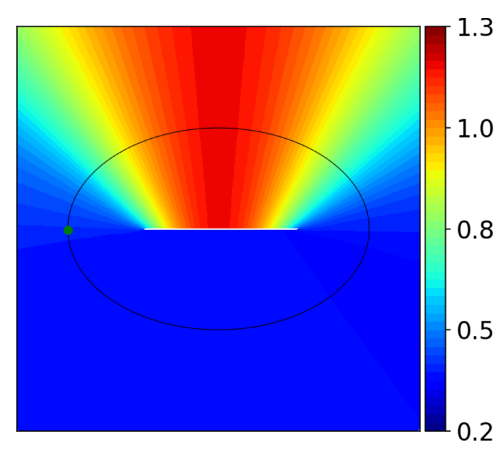

(A)

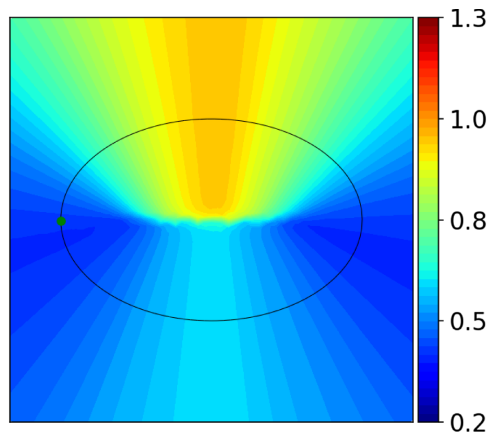

(B)

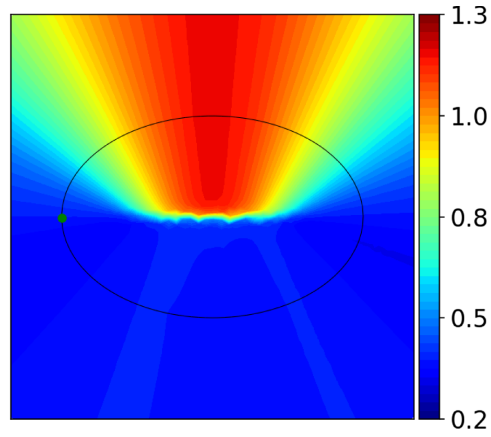

(C)

Figure 10. The numerical solution $u$ of the variational problem (2.5) extended constantly along rays (for visualization purpose). Inaccuracies occur if the skeleton $\Sigma$ is not removed from the mesh or if the weight $\omega$ does not vanish in its vicinity. (A) Mesh $\mathcal{T}^{\prime}$ (skeleton manually truncated), $\omega=1$. (B) Mesh $\mathcal{T}, \omega=1$. (C) Mesh $\mathcal{T}, \omega=2 /\left(1+\left|d_{\Omega}\right|^{3.5}\left|\Delta d_{\Omega}\right|^{3.5}\right)$.

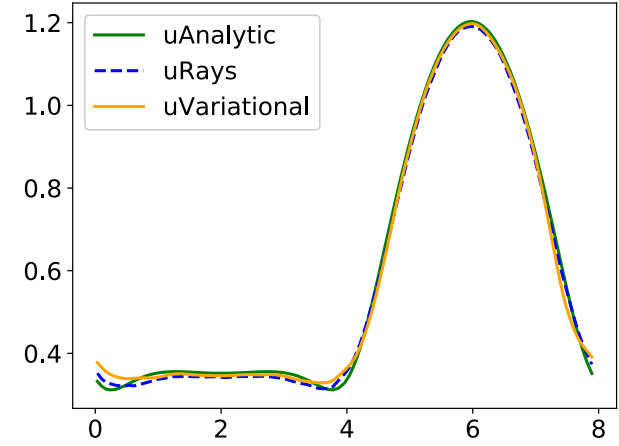

(A)

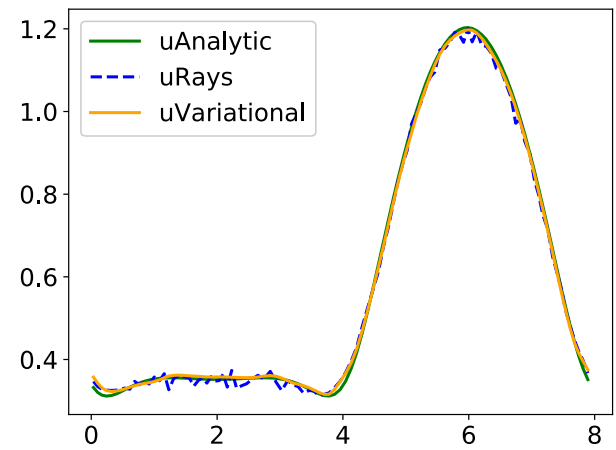

(C)

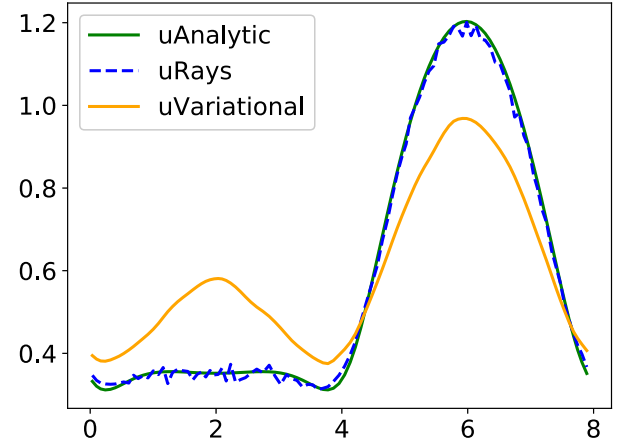

(B)

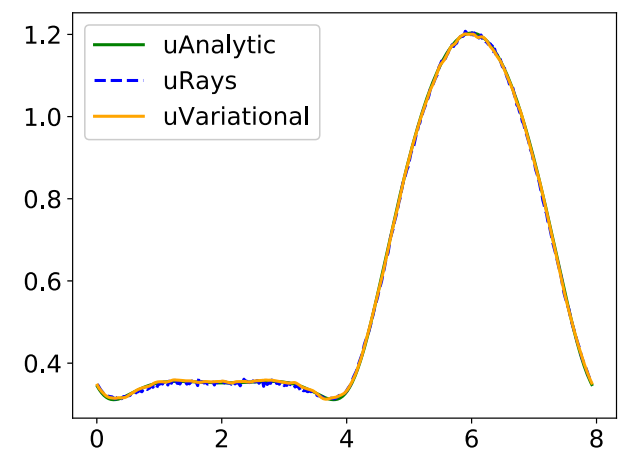

(D)

FiguRE 11. Comparison between the results of a direct integration along rays and our variational method for the example of Section 3.4.2. (A) Mesh $\mathcal{T}^{\prime}, \omega=1$ (B) Mesh $\mathcal{T}, \omega=1$ (C) Mesh $\mathcal{T}, \omega=2 /\left(1+\left|d_{\Omega}\right|^{3.5}\left|\Delta d_{\Omega}\right|^{3.5}\right)$. (D) Mesh $\mathcal{T}, \omega=2 /\left(1+\left|d_{\Omega}\right|^{3.5}\left|\Delta d_{\Omega}\right|^{3.5}\right.$ ) (Fine mesh of Fig. $4 \mathrm{C})$. 

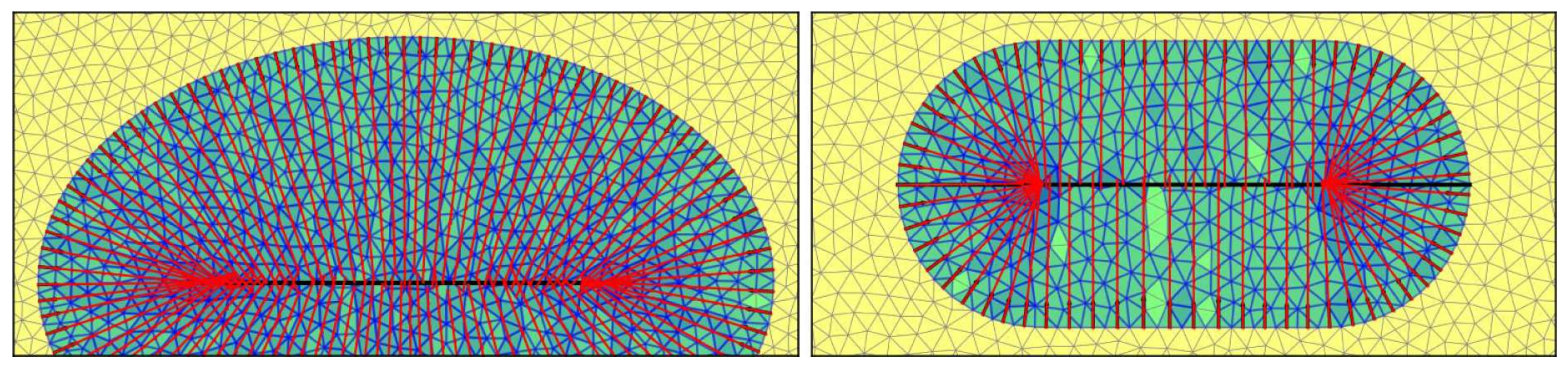

FiguRE 12. Numerical rays for the example of Sections 3.4.2 and 3.4.3. The inaccuracy of the direct integration along rays is related to that of the skeleton detection within the last triangle. The triangle paths are colored in transparent blue (the darker, the more often visited); depicting that some mesh triangles might not be crossed by all the rays or some triangles may be visited more often than others. (Color online).

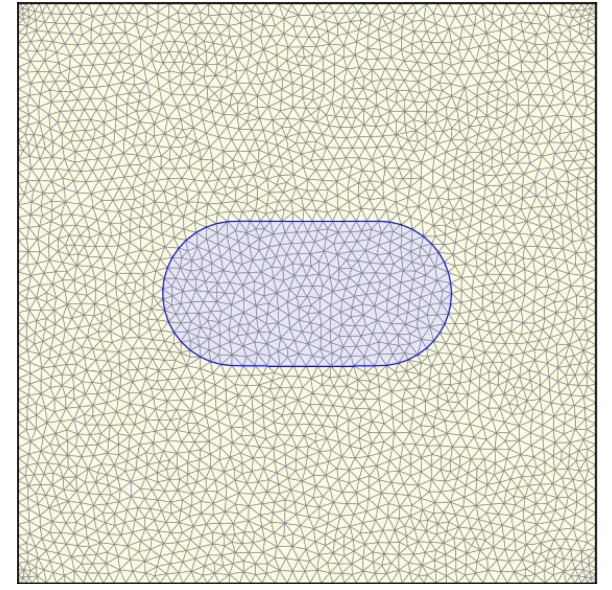

(A)

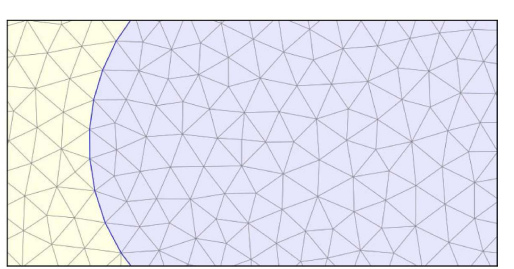

(B)

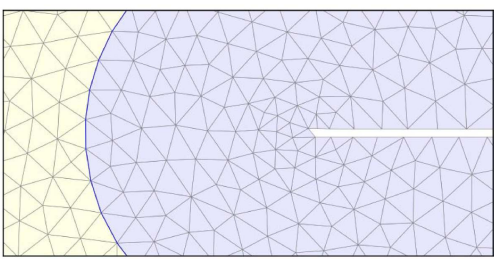

(C)

Figure 13. Meshes used for the test-case of Section 3.4.3. (A) Mesh $\mathcal{T}$ of the bulk domain $D$ with $\Omega \subset D$ (in blue) discretized as a submesh. (B) Zoom on the mesh $\mathcal{T}$. (c) Zoom on the mesh $\mathcal{T}^{\prime}$ (skeleton $\Sigma$ truncated). (Color online).

In particular, $u$ is ill-defined at the points $x \in \partial \Omega$ where the curvature is discontinuous.

Both methods for the numerical evaluation of $u$ (i.e. the direct integration along rays and our variational method) are applied, and the results are represented on Figure 14. Even though this example does not fit into the admissible setting of Section 2, the results indicate that our numerical method still works, up to over-smoothing inaccuracies of the computed solution $u$ near the discontinuities of the curvature $\kappa$.

\subsubsection{A test case involving a Lipschitz domain $\Omega$ with angular corners}

In this last example, we consider the following situation:

$$
D=\left\{\left(x_{1}, x_{2}\right) \in \mathbb{R}^{2}|| x_{1} \mid \leq 2 \text { and }|y| \leq 2\right\}, \Omega=\left\{\left(x_{1}, x_{2}\right) \in D \mid \max \left(x_{1}^{2} / 2-x_{2}-1, x_{2}\right) \leq 0\right\},
$$

together with the datum function $f$ :

$$
f\left(x_{1}, x_{2}\right)=\cos \left(6 x_{1}\right)^{2},
$$




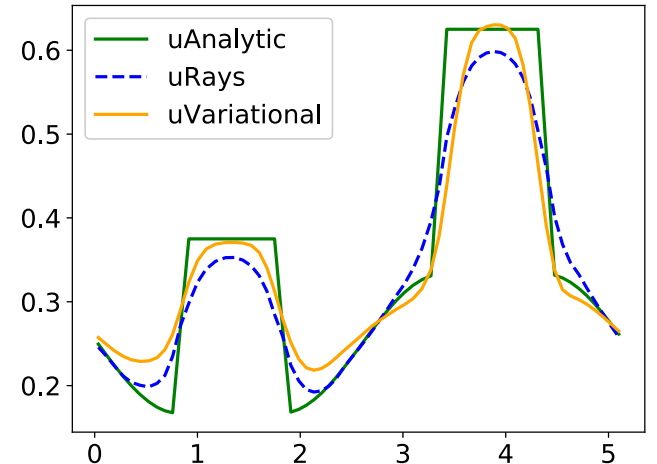

(A)

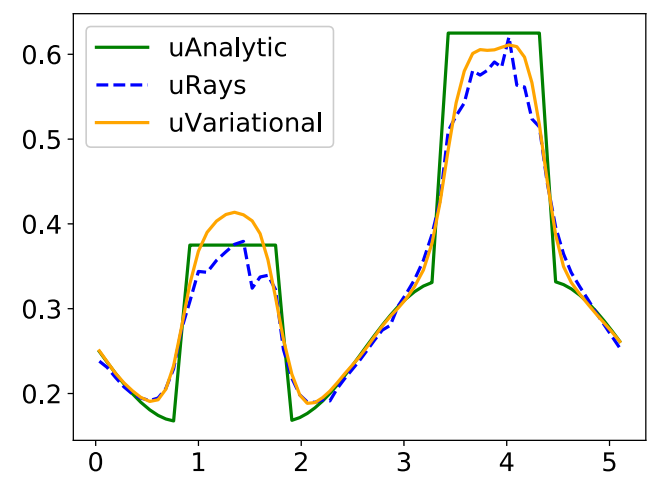

(C)

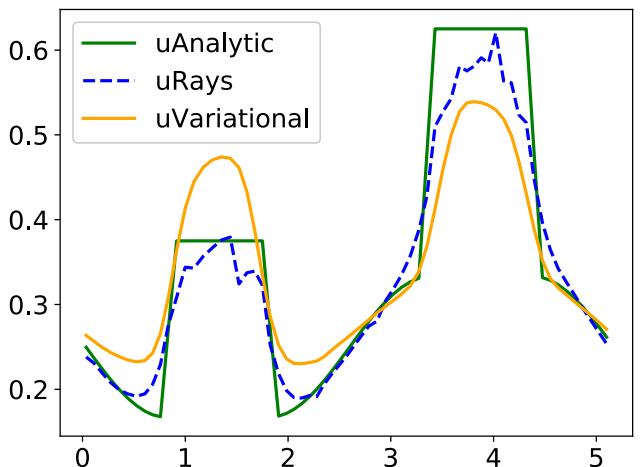

(B)

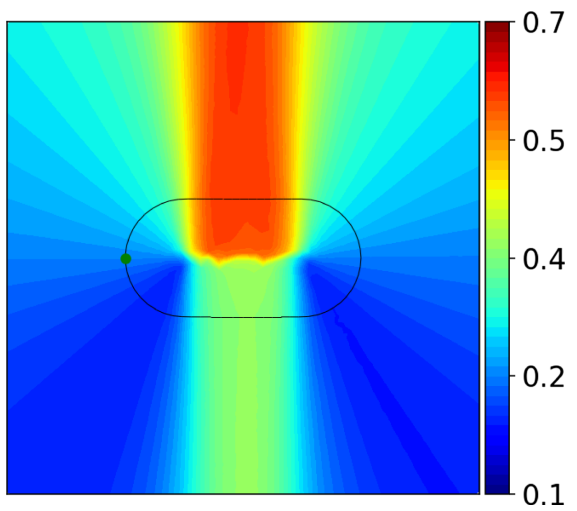

(D)

FIgURE 14. Comparison between the direct integration along rays and our variational method for the example of Section 3.4.3. (A) Mesh $\mathcal{T}^{\prime}, \omega=1$. (B) Mesh $\mathcal{T}, \omega=1$. (C) Mesh $\mathcal{T}, \omega=$ $2 /\left(1+\left|d_{\Omega}\right|^{3.5}\left|\Delta d_{\Omega}\right|^{3.5}\right)$. (D) Variational solution $u$ on the mesh $\mathcal{T}, \omega=2 /\left(1+\left|d_{\Omega}\right|^{3.5}\left|\Delta d_{\Omega}\right|^{3.5}\right)$ extended constantly along rays. (Color online).

as illustrated on Figure 15. Again, the theoretical framework of iii 2 does not apply to the present situation because:

- The vector field $\nabla d_{\Omega}$ is not of class $\mathcal{C}^{1}$ on $U$ and is not $U$-filling: the reunion of all the rays emerging from points $y \in \partial \Omega$ does not cover the whole set $U=D \backslash \bar{\Sigma}$;

- The flow $\eta$ of this vector field is not of class $\mathcal{C}^{1}$, because the curvature $\kappa$ of the boundary (and even the normal vector) is discontinuous at the corners. Numerically, $\left|\Delta d_{\Omega}\right|$ blows up in the whole area filled by the skeleton and the set of points that are not covered by normal rays.

Still, the quantity $u$ given by (2.6) can be calculated analytically wherever it makes sense. A few elementary, albeit technical computations yield:

$$
u\left(y_{1}, y_{2}\right)=\left\{\begin{array}{lll}
\cos \left(6 y_{1}\right)^{2}\left(2-\lambda_{-}\left(s^{-1}\left(y_{1}\right)\right)\right) & \text { if } & y_{2} \geq 0 \\
\phi\left(\lambda_{+}\left(y_{1}\right)\right)-\phi\left(\lambda_{-}\left(y_{1}\right)\right) & \text { if } & y_{2} \leq 0
\end{array}\right.
$$

where

$$
\lambda_{-}(t)=\frac{t^{2} / 2-1}{1+1 / \sqrt{1+t^{2}}}, \lambda_{+}(t)=\min \left((2 /|t|-1), t^{2} / 2\right) \sqrt{1+t^{2}},
$$




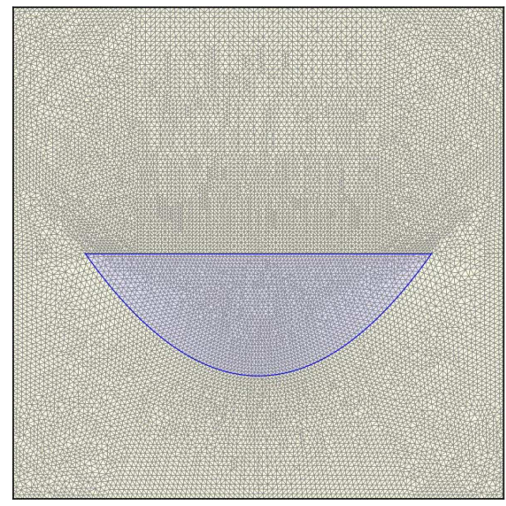

(A)

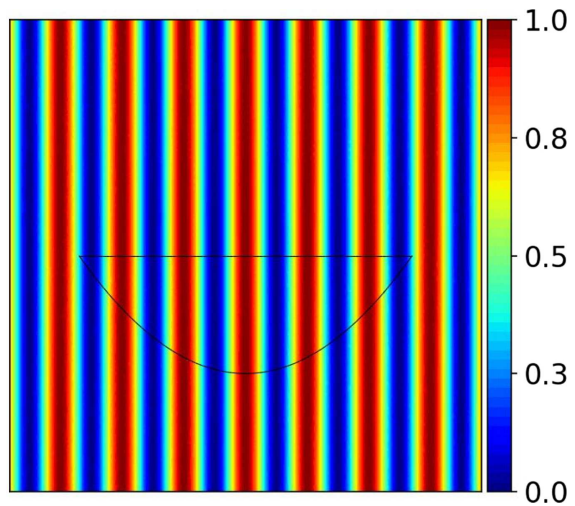

(B)

Figure 15. Setting for the example of Section 3.4.4. (A) The numerical mesh $\mathcal{T}$ for $D(\Omega$ in blue). (B) The numerical function $f$. (Color online).

$s^{-1}$ is the reciprocal function of $t \mapsto s(t)=\left(1+\frac{\lambda_{-}(t)}{\sqrt{1+t^{2}}}\right) t$ and $\phi$ is a primitive function of $\lambda \mapsto \cos (6 t(1+$ $\left.\left.\lambda / \sqrt{1+t^{2}}\right)\right)^{2}\left(1+\lambda /\left(1+t^{2}\right)^{3 / 2}\right)$.

A numerical approximation to this function $u$ is computed by using either a direct integration along rays or our variational method based on (2.5), on a single mesh $\mathcal{T}$ where the skeleton $\Sigma$ is not removed, and using three possible choices of the weight $\omega$. The results are represented on Figure 16. In all cases, the observed numerical inaccuracies, characterized by very high values, are concentrated near the angular corners of $\Omega$, while the method yields satisfying accuracy on the remaining smooth parts of $\partial \Omega$. Again, we observe the poor accuracy associated to the choice of a constant weight $\omega=1$. For this example, a uniformly small weight $\omega=1 e^{-10}$ seems to yield a better accuracy than the previous choice $\omega=2 /\left(1+\left|d_{\Omega}\right|^{3.5}\left|\Delta d_{\Omega}\right|^{3.5}\right)$.

\section{Applichtions to maximum and minimum thickness COnstraints in Shape OPTIMIZATION}

In the shape optimization setting which is the original motivation for our work, we show how the general method proposed in Section 2 and the inferred numerical methods in Section 3 allow to efficiently implement geometric constraints, namely maximum and minimum thickness constraints. Here no comparison is made between our new variational approach and the previous method (using direct integration along rays) to evaluate the shape derivatives of the related shape functionals. The optimized shapes and topologies resulting from the variational method are very similar to those obtained in the previous works $[7,50]$. There is no clear gain in computational time but there is a very substantial simplification of the implementation (which would be tremendous in $3-d)$.

\subsection{Shape optimization of linearly elastic structures}

We consider shapes, that is, smooth bounded domains $\Omega$ enclosed in a fixed, bounded and Lipschitz hold-all domain $D \subset \mathbb{R}^{n}$. Any such shape $\Omega$ is clamped on a part $\Gamma_{D}$ of its boundary, and traction loads $\boldsymbol{g} \in L^{2}\left(\Gamma_{N}\right)$ are applied on a disjoint region of $\partial \Omega$; the complement $\Gamma:=\partial \Omega \backslash\left(\overline{\Gamma_{D}} \cup \overline{\Gamma_{N}}\right)$ is traction-free and body forces are omitted for simplicity; $\Gamma$ is the only region of the boundary $\partial \Omega$ which is subject to optimization. 


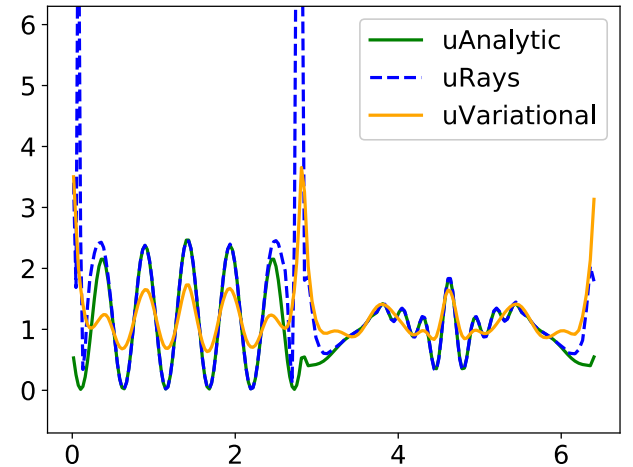

(A)

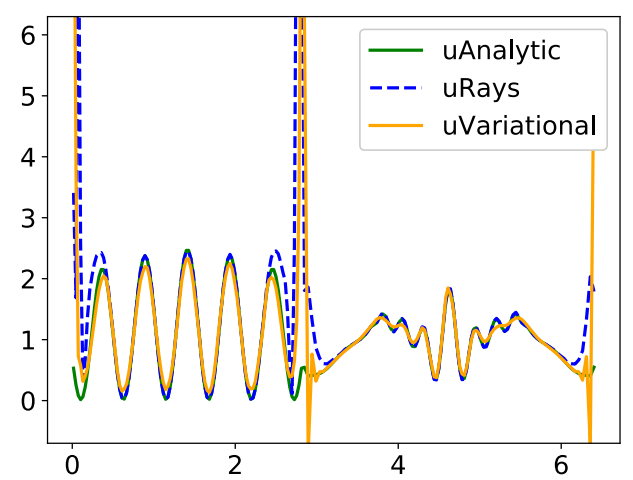

(c)

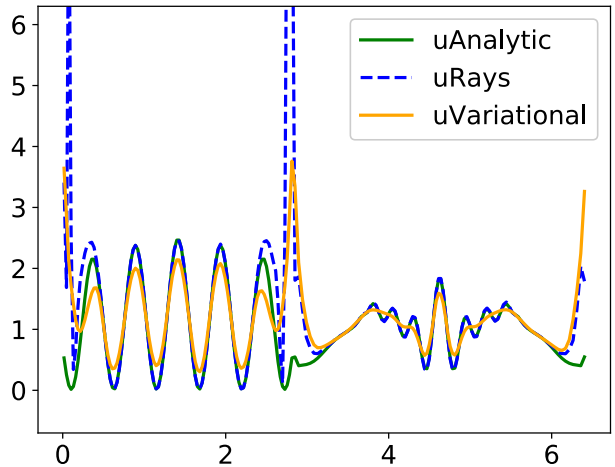

(B)

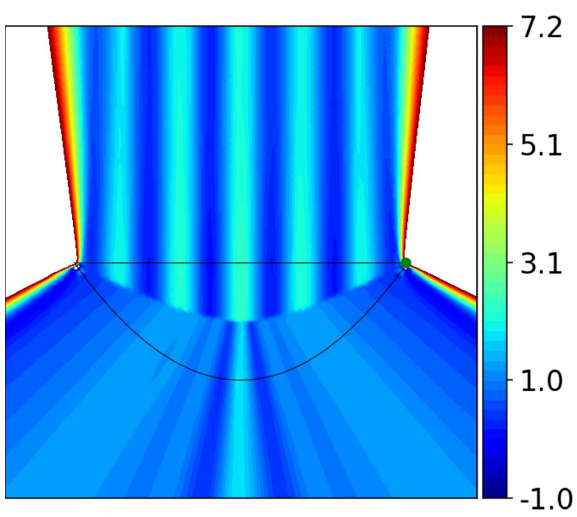

(D)

FiguRE 16. Comparison between direct integration along normal rays and our variational method for the example of Section 3.4.4. (A) $\omega=1$. (B) $\omega=2 /\left(1+\left|d_{\Omega}\right|^{3.5}\left|\Delta d_{\Omega}\right|^{3.5}\right.$ ). (C) $\omega=1 e-10$. (D) Numerical solution $u$ obtained with $\omega=1 e-10$ (extended along rays with (3.25)). (Color online).

In this situation, the displacement $\boldsymbol{u}_{\Omega}$ of the shape is characterized as the unique solution in $H^{1}\left(\Omega, \mathbb{R}^{n}\right)$ to the linearized elasticity system:

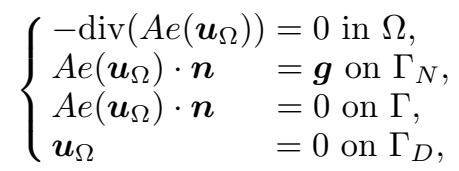

where $e(\boldsymbol{u}):=\frac{1}{2}\left(\nabla \boldsymbol{u}+\nabla \boldsymbol{u}^{T}\right)$ is the strain tensor associated to the displacement $\boldsymbol{u}$ and $A$ is the Hooke's law, defined for any symmetric $n \times n$ matrix by

$$
A e(\boldsymbol{u})=2 \mu e(\boldsymbol{u})+\lambda \operatorname{Tr}(e(\boldsymbol{u})) \mathrm{I},
$$

involving the Lamé coefficients $\lambda, \mu$ which characterize the physical properties of the constituent material.

In this context, we consider structural optimization problems of the form

$$
\min _{\Omega \subset D} J(\Omega) \text {, s.t. } P(\Omega) \leq 0,
$$


where $J(\Omega)$ is a performance criterion, which will typically be the volume $\operatorname{Vol}(\Omega)$ or the compliance $C(\Omega)$ of shapes:

$$
\operatorname{Vol}(\Omega)=\int_{\Omega} \mathrm{d} x, C(\Omega)=\int_{\Omega} A e\left(\boldsymbol{u}_{\Omega}\right): e\left(\boldsymbol{u}_{\Omega}\right) \mathrm{d} x,
$$

and $P(\Omega)$ is a geometric constraint

$$
P(\Omega)=\int_{D} j\left(d_{\Omega}\right) \mathrm{d} x
$$

involving the signed distance function $d_{\Omega}$ to $\Omega$ and a given smooth function $j: \mathbb{R} \rightarrow \mathbb{R}$.

In order to achieve the numerical resolution of (4.2), we rely on Hadamard's method of boundary variations (see for instance $[1,3,40,51,58]$ ), whereby variations of a given shape $\Omega$ are considered under the form

$$
\Omega_{\theta}:=(\operatorname{Id}+\theta)(\Omega), \theta \in W^{1, \infty}\left(D, \mathbb{R}^{n}\right),\|\theta\|_{W^{1, \infty}\left(D, \mathbb{R}^{n}\right)}<1 .
$$

Definition 4.1. A function $F(\Omega)$ of the domain is shape differentiable at a shape $\Omega$ if the underlying mapping $\theta \mapsto F\left(\Omega_{\theta}\right)$, from $W^{1, \infty}\left(D, \mathbb{R}^{n}\right)$ into $\mathbb{R}$, is Fréchet differentiable at $\theta=0$. The corresponding derivative $\theta \mapsto$ $F^{\prime}(\Omega)(\theta)$ is the shape derivative of $F$ at $\Omega$ and the following expansion holds in the vicinity of $\theta=0$ :

$$
F\left(\Omega_{\theta}\right)=F(\Omega)+F^{\prime}(\Omega)(\theta)+o(\theta), \text { where } \frac{|o(\theta)|}{\|\theta\|_{W^{1, \infty}\left(D, \mathbb{R}^{n}\right)}} \stackrel{\theta \rightarrow 0}{\longrightarrow} 0 .
$$

Remark 4.2. In our setting, the set of admissible deformation fields $\theta$ used in (4.5) is restricted to smooth vector fields, which vanish on the non optimizable subset $\Gamma_{N}$ and $\Gamma_{D}$ of the boundaries of shapes.

The shapes derivatives of the volume and compliance functions defined in (4.3) are classically given by (see for instance [2]):

$$
\operatorname{Vol}^{\prime}(\Omega)(\theta)=\int_{\Gamma} \theta \cdot \boldsymbol{n} \mathrm{d} y, \text { and } C^{\prime}(\Omega)(\theta)=-\int_{\Gamma} A e\left(\boldsymbol{u}_{\Omega}\right): e\left(\boldsymbol{u}_{\Omega}\right)(\theta \cdot \boldsymbol{n}) \mathrm{d} y .
$$

where we recall that $\mathrm{d} y$ stands for the surface measure on $\Gamma$. As far as the shape derivative of the geometric constraint $P(\Omega)$ in (4.4) is concerned, the following (equivalent) surface and volumetric expressions were obtained in our previous work [5]:

$$
P^{\prime}(\Omega)(\theta)=\int_{D \backslash \bar{\Sigma}} j^{\prime}\left(d_{\Omega}(x)\right) d_{\Omega}^{\prime}(\theta)(x) \mathrm{d} x=\int_{\Gamma} u \theta \cdot \boldsymbol{n} \mathrm{d} y
$$

where the "Eulerian" derivative $d_{\Omega}^{\prime}(\theta)$ of the signed distance function $d_{\Omega}$ is defined on $U=D \backslash \bar{\Sigma}$ by:

$$
\forall x \in D \backslash \bar{\Sigma}, d_{\Omega}^{\prime}(\theta)(x)=-\theta\left(p_{\partial \Omega}(x)\right) \cdot \boldsymbol{n}\left(p_{\partial \Omega}(x)\right),
$$

and the scalar function $u: \Gamma \rightarrow \mathbb{R}$ reads:

$$
u(y)=-\int_{s \in \operatorname{ray}(y)} j^{\prime}\left(d_{\Omega}(s)\right) \prod_{1 \leq i \leq n-1}\left(1+\kappa_{i}(y) d_{\Omega}(s)\right) \mathrm{d} s,
$$

as follows from an application of the coarea formula with the help of the material recalled in Section 3.1.

Using the conclusions of Section 2, the function $u$ in (4.7) may be conveniently evaluated by solving the variational problem:

$$
\text { Find } u \in V_{\omega} \text { such that } \forall v \in V_{\omega}, \int_{\partial \Omega} u v \mathrm{~d} y+\int_{D \backslash \bar{\Sigma}} \omega\left(\nabla d_{\Omega} \cdot \nabla u\right)\left(\nabla d_{\Omega} \cdot \nabla v\right) \mathrm{d} x=-\int_{D \backslash \bar{\Sigma}} j^{\prime}\left(d_{\Omega}(x)\right) v \mathrm{~d} x,
$$




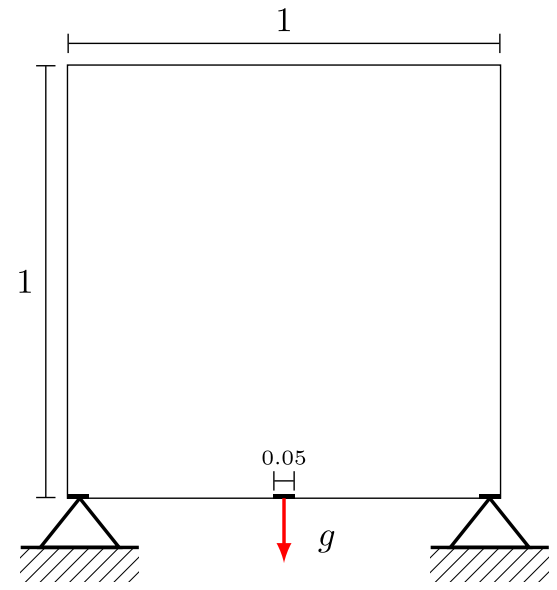

(A)

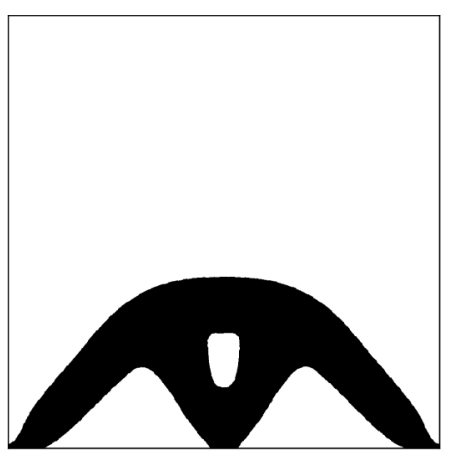

(B)

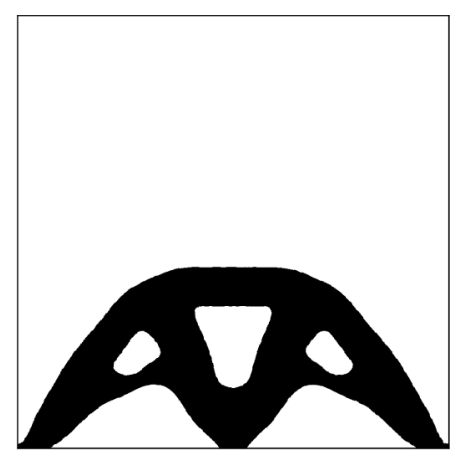

(C)

Figure 17. Physical setting and optimized shapes obtained in the two-dimensional arch optimization test-case of Section 4.2.1. (A) Setting of the optimization problem. (B) Optimized shape without maximum thickness constraint $\left(\max \left|d_{\Omega}\right|=0.10\right)$. (C) Optimized shape with maximum thickness constraint $\left(\max \left|d_{\Omega}\right|=0.07\right)$.

for a suitable weight $\omega$ satisfying (H1)-(H3), as discussed in Section 3. Note how easily (4.7) is retrieved by taking the test function $v=-d_{\Omega}^{\prime}(\theta)$ in (4.9). Since $\theta \cdot \boldsymbol{n} \in L^{\infty}(\partial \Omega)$, the derivative given by (4.8) is indeed an admissible test function $d_{\Omega}^{\prime}(\theta) \in V_{\omega}$, for it belongs to $L^{\infty}(D \backslash \bar{\Sigma})$ and is constant along normal rays.

In our numerical implementation, we set $\omega=1 /\left(1+100\left|d_{\Omega} \Delta d_{\Omega}\right|^{3.5}\right)$ in order to solve (4.9) with $\mathbb{P}_{1}$ finite elements, the constant 100 being selected to increase the slope of the weight near the skeleton.

Let us now outline briefly the numerical implementation of the above shape optimization framework. All the involved finite element computations are performed within the FreeFem++ environment [39] - whether they are related to the resolution of the physical system (4.1) or to that of our variational problem (4.9). The resolution of the constrained optimization program (4.2) is carried out by the discretization of a specific gradient flow in the spirit of [56], see the details of our algorithm in our recent work [37]. When it comes to representing shapes and their evolution in the course of the iterative resolution of (4.2), the level set based mesh evolution method of $[4,36]$ is used, as a convenient combination of the "classical" level set method for shape and topology optimization $[2,61]$ and the mmg open-source mesh modification algorithm [21].

\subsection{Shape optimization under a maximum thickness constraint}

We first consider structural optimization problems featuring a maximum thickness constraint. Following the work in $[7,50]$, a shape $\Omega \subset D$ is said to have maximum thickness lower than $d_{\max }>0$ provided:

$$
\forall x \in \Omega, d_{\Omega}(x) \geq-d_{\max } / 2 .
$$

Loosely speaking, this amounts to saying that the skeleton $\Sigma$ of $\Omega$ lies at a distance of at most $d_{\max }$ from the boundary $\partial \Omega$. Following closely $[7,50]$, the pointwise constraint (4.10) is relaxed into a single integral constraint 


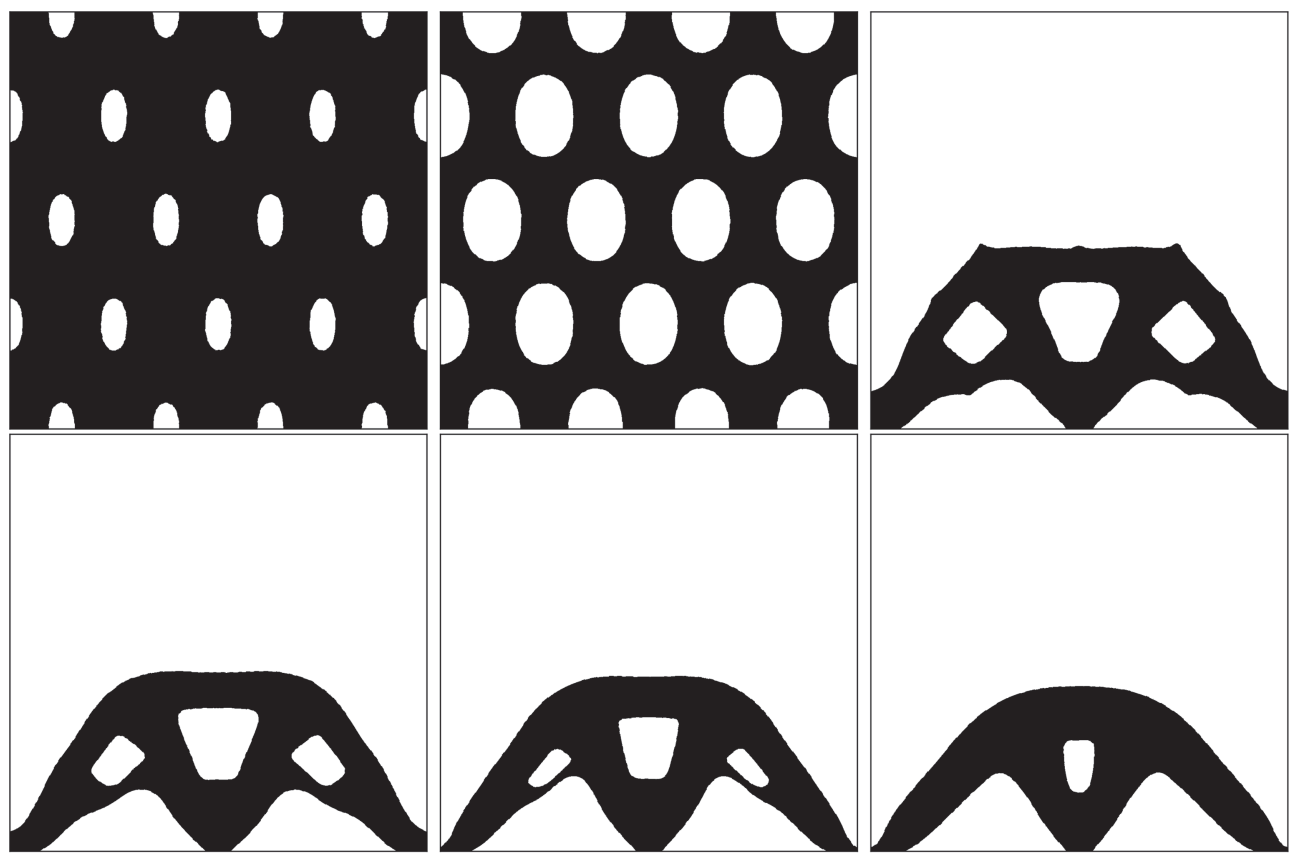

(A)

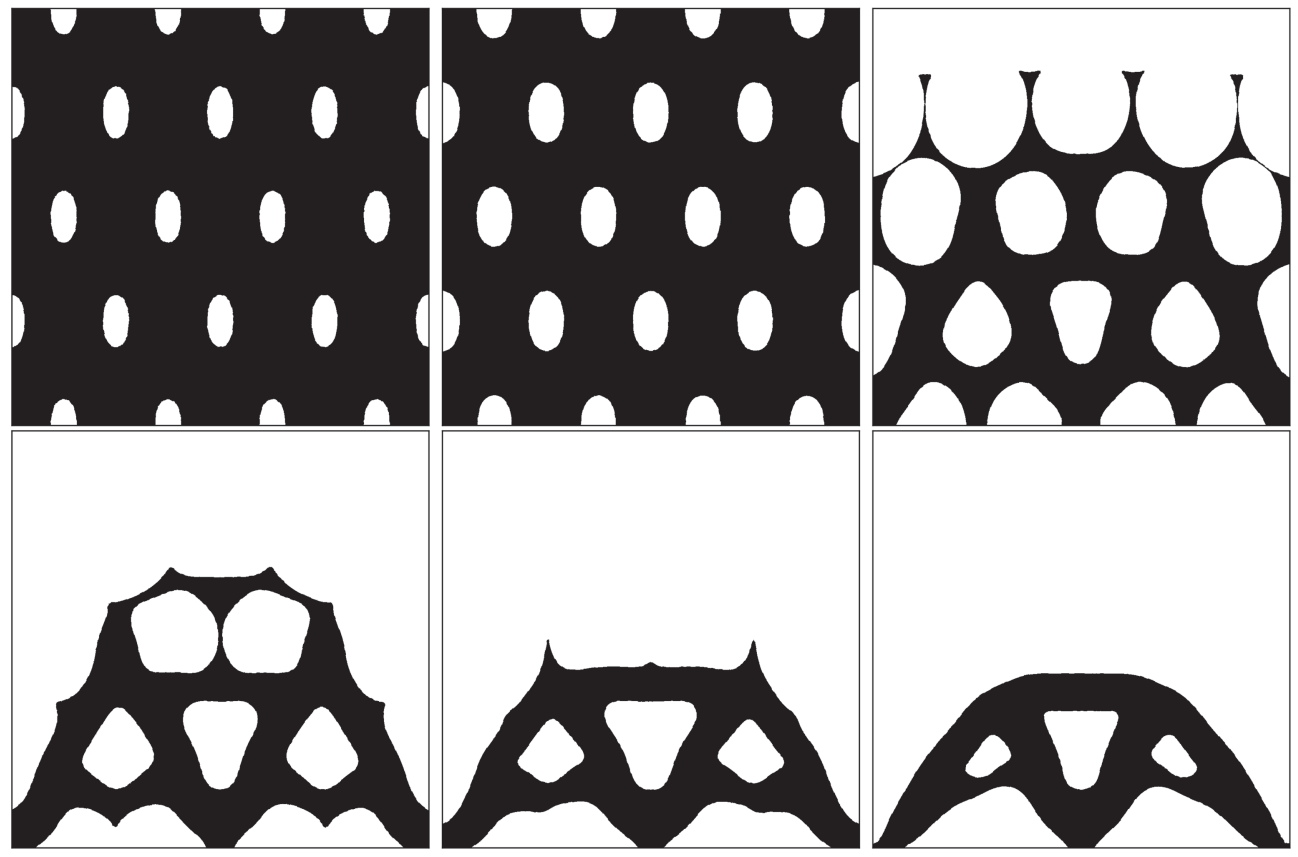

(B)

FiguRE 18. Iterations $0,5,40,65,100$ and 200 in the shape optimization example of a 2-d arch of Section 4.2.1. (A) Without thickness constraint. (B) With maximum thickness constraint. 


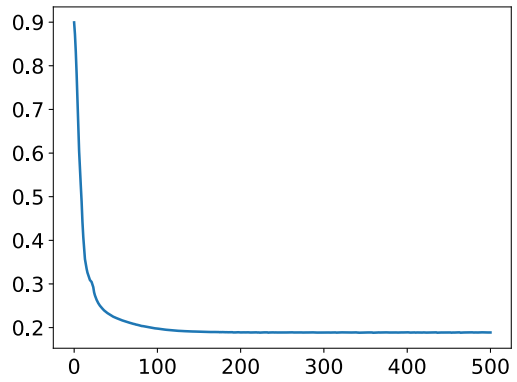

(A)

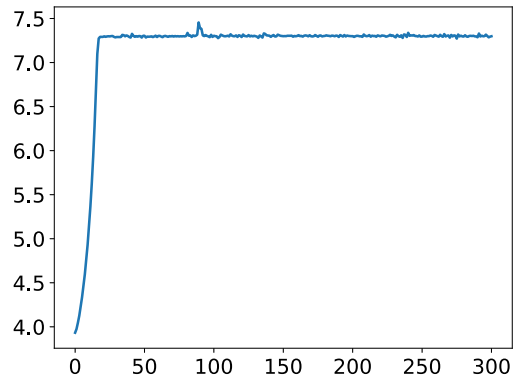

(B)

FIgURE 19. Optimization histories for the optimization problem (4.14) without maximum thickness constraint. Final values: volume $\operatorname{Vol}(\Omega)=0.19$, and compliance $C(\Omega)=7.00$. (A) Volume $\operatorname{Vol}(\Omega)$. (B) Compliance $C(\Omega)$.

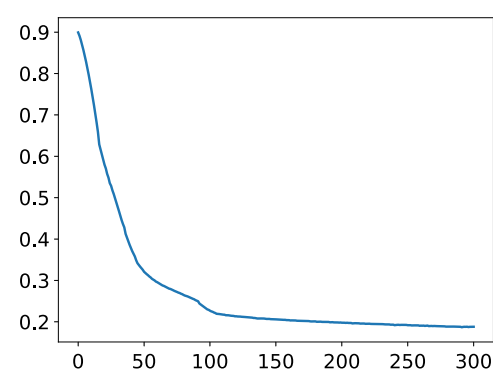

(A)

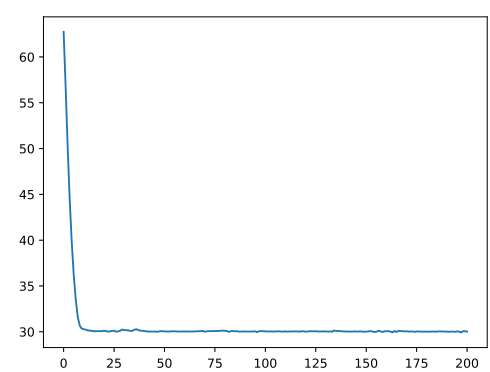

(в)

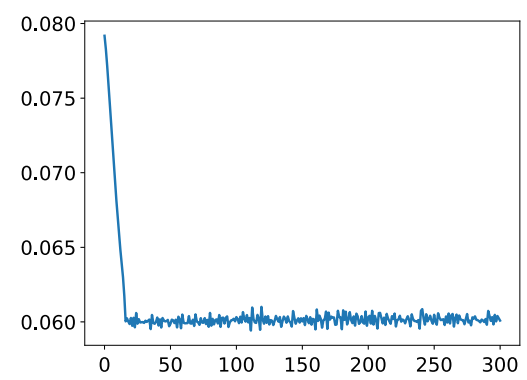

(c)

FiguRE 20. Optimization histories for the optimization problem (4.14) with maximum thickness constraint. Final values: volume $\operatorname{Vol}(\Omega)=0.19$, compliance $C(\Omega)=7.30$, and penalty $P_{\mathrm{MaxT}}(\Omega)=7.30$. (A) Volume $\operatorname{Vol}(\Omega)$. (B) Compliance $C(\Omega)$. (C) Thickness penalty $P_{\mathrm{MaxT}}(\Omega)$.

formulated in terms of the following penalty functional $P_{\operatorname{MaxT}}(\Omega)$ :

$$
P_{\mathrm{MaxT}}(\Omega) \leq \frac{d_{\max }}{2}, \text { where } P_{\mathrm{MaxT}}(\Omega):=\left(\frac{\int_{\Omega} h\left(d_{\Omega}\right) d_{\Omega}^{2} \mathrm{~d} x}{\int_{\Omega} h\left(d_{\Omega}\right) \mathrm{d} x}\right)^{1 / 2},
$$

and $h$ is a regularized Heaviside function centered at $d_{\max } / 2$ :

$$
\forall x \in \mathbb{R}, h(x)=\frac{1}{2}\left(1+\tanh \left(\frac{x-d_{\max } / 2}{\alpha_{f} d_{\max } / 2}\right)\right) .
$$

The parameter $\alpha_{f}$ in (4.12) tunes the level of regularization; in our context, it is set to $\alpha_{f}=\frac{4 h_{\max }}{5 d_{\max }}$, where $h_{\max }$ is the maximum edge length of the computational mesh of $D$. 


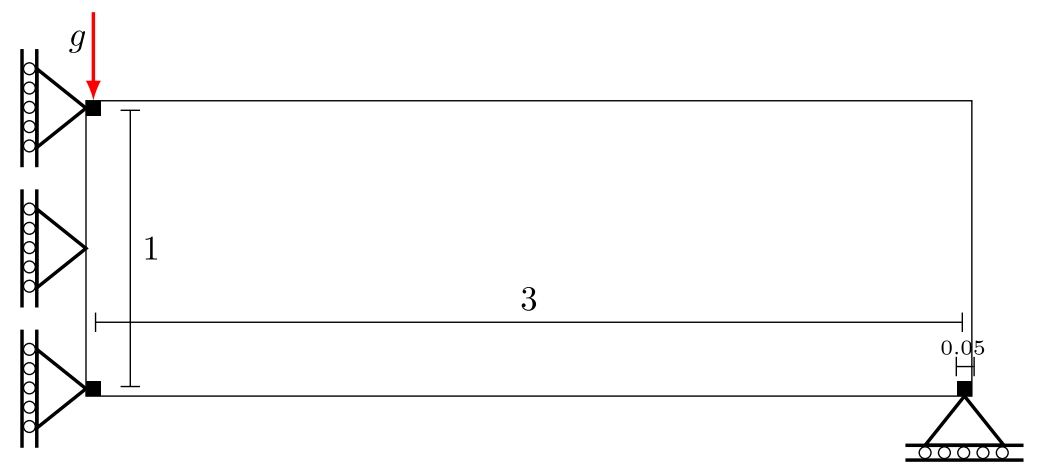

(A)

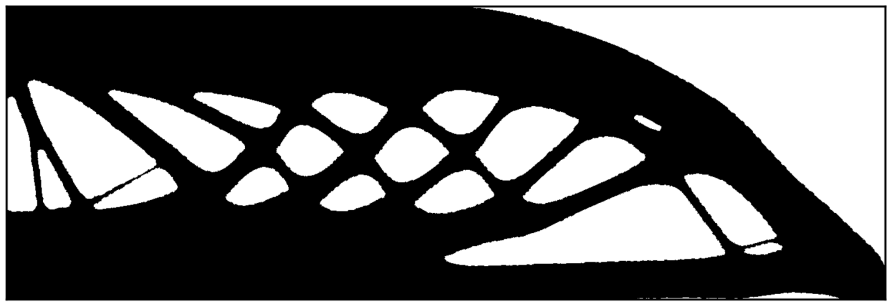

(B)

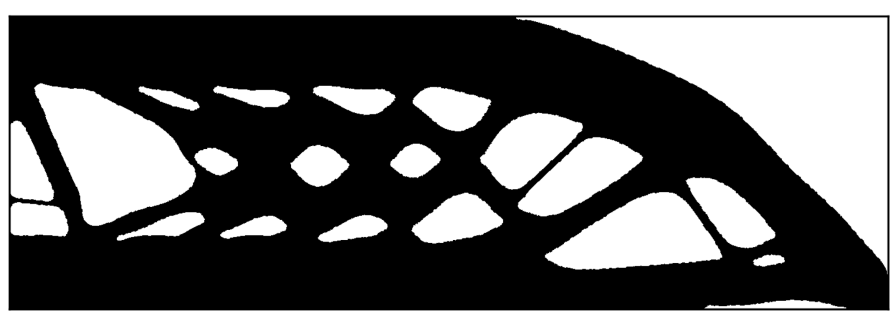

(C)

FiguRE 21. Physical setting and obtained optimized shapes in the 2-d MBB beam optimization test-case of Section 4.2.2 $\left(\max \left|d_{\Omega}\right|=0.26\right)$. (A) Setting of the optimization problem. Small black areas correspond to non optimizable parts of the design domain. (B) Optimized shape without maximum thickness constraint $\left(\max d_{\Omega}=0.36\right)$. (c) Optimized shape with maximum thickness constraint.

A simple calculation yields the shape derivative of $P_{\operatorname{MaxT}}(\Omega)$ :

$$
\begin{aligned}
P_{\text {MaxT }}^{\prime}(\Omega)(\theta)=-\frac{1}{2 P_{\operatorname{MaxT}}(\Omega)} \frac{\int_{\Omega} h\left(d_{\Omega}\right) d_{\Omega}^{2} \mathrm{~d} x}{\left(\int_{\Omega} h\left(d_{\Omega}\right) \mathrm{d} x\right)^{2} h(0) \int_{\Gamma} \theta \cdot \boldsymbol{n} \mathrm{d} y} \\
+\frac{1}{2 P_{\operatorname{MaxT}}(\Omega)} \int_{\Omega}\left[\frac{h^{\prime}\left(d_{\Omega}\right) d_{\Omega}^{2}+2 h\left(d_{\Omega}\right) d_{\Omega}}{\int_{\Omega} h\left(d_{\Omega}\right) \mathrm{d} z}-\frac{\int_{\Omega} h\left(d_{\Omega}\right) d_{\Omega}^{2} \mathrm{~d} z}{\left.\left(\int_{\Omega} h\left(d_{\Omega}\right) \mathrm{d} z\right)^{2} h^{\prime}\left(d_{\Omega}\right)\right]} d_{\Omega}^{\prime}(\theta) \mathrm{d} x\right.
\end{aligned}
$$




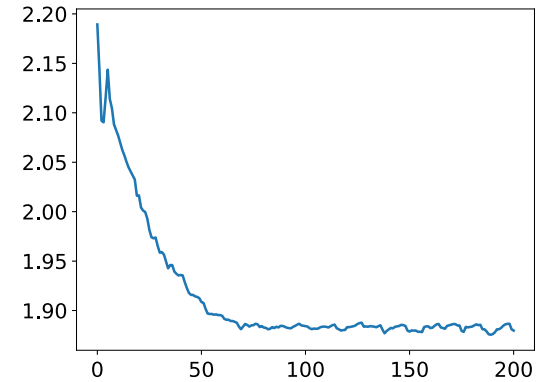

(A)

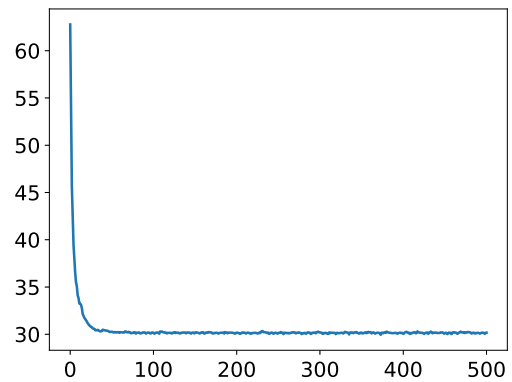

(B)

FIgURE 22. Optimization histories for the shape optimization problem (4.14) of the 2-d MBBBeam of Section 4.2.2 without including a maximum thickness constraint. Final values: volume $\operatorname{Vol}(\Omega)=1.88$, and compliance $C(\Omega)=29.96$. (A) Volume $\operatorname{Vol}(\Omega)$ (B) Compliance $C(\Omega)$.

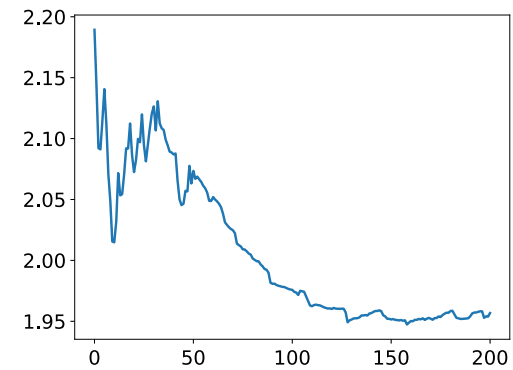

(A)

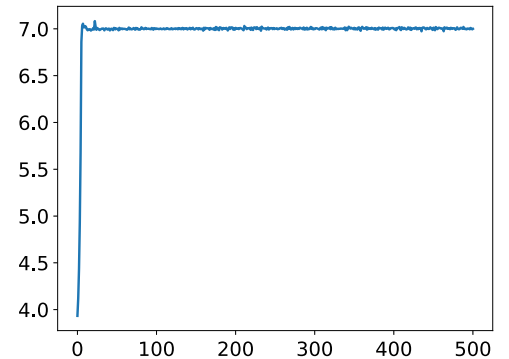

(B)

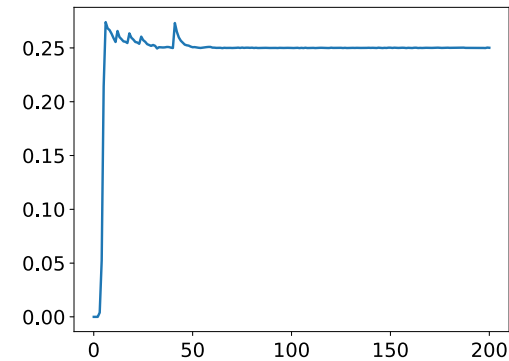

(C)

FiguRE 23. Optimization histories for the shape optimization problem (4.14) of the 2-d MBBBeam of Section 4.2.2 with maximum thickness constraint. Final values: $\operatorname{Vol}(\Omega)=1.96$, compliance: $C(\Omega)=30.03$, and penalty $P_{\mathrm{MaxT}}(\Omega)=0.25$. (A) Volume $\operatorname{Vol}(\Omega)$. (B) Compliance $C(\Omega)$. (C) Thickness Penalty $P_{\mathrm{MaxT}}(\Omega)$.

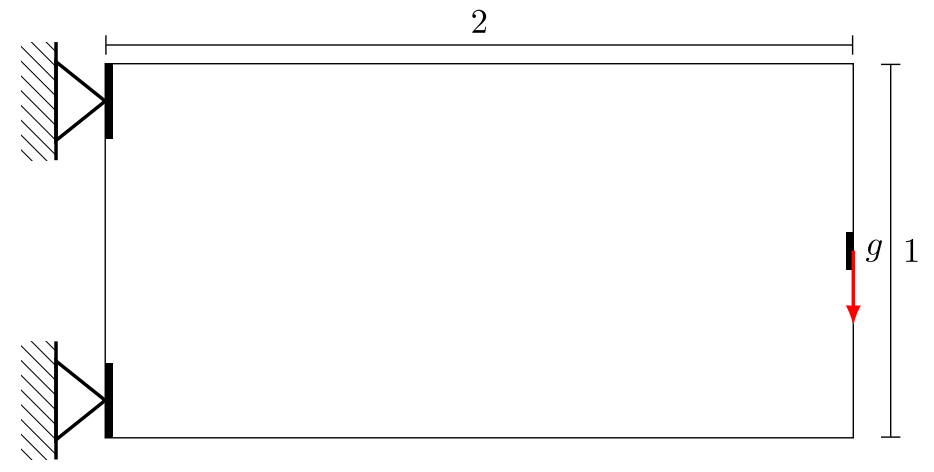

FiguRE 24. Setting of the cantilever test case of Section 4.3.1. 


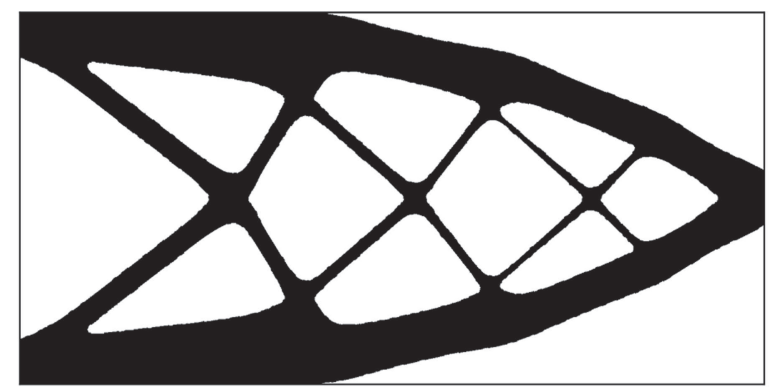

(A)

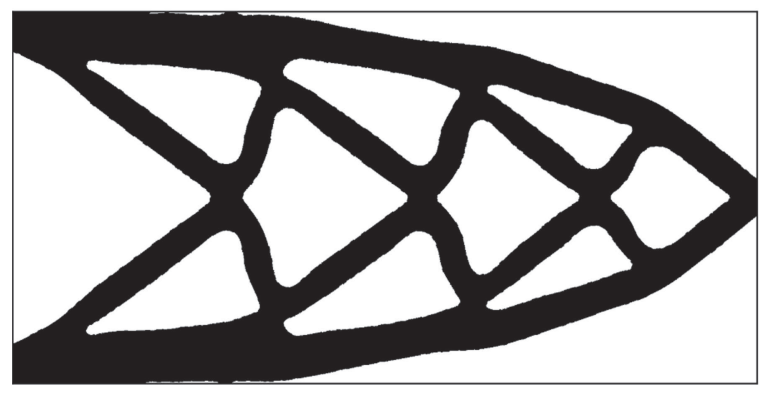

(B)

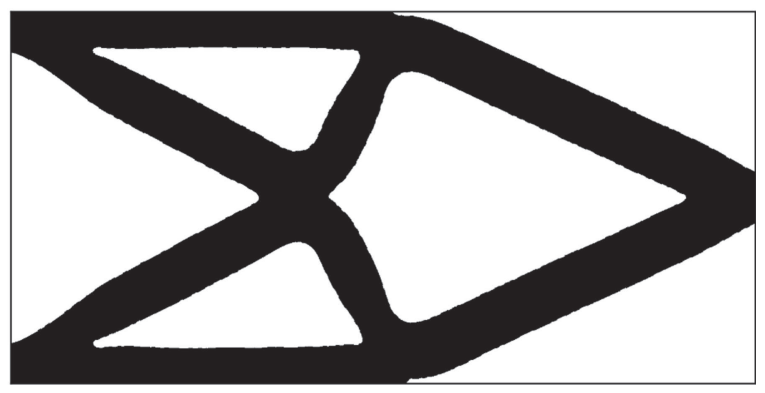

(C)

Figure 25. Optimization of the shape of the 2-d cantilever of Section 4.3.1 under minimum thickness constraint. (A) Optimized shape without including minimum thickness constraint. (B) Optimized shape with minimum thickness constraint $\left(d_{\min }=0.1\right)$. (C) Optimized shape with minimum thickness constraint $\left(d_{\min }=0.2\right)$.

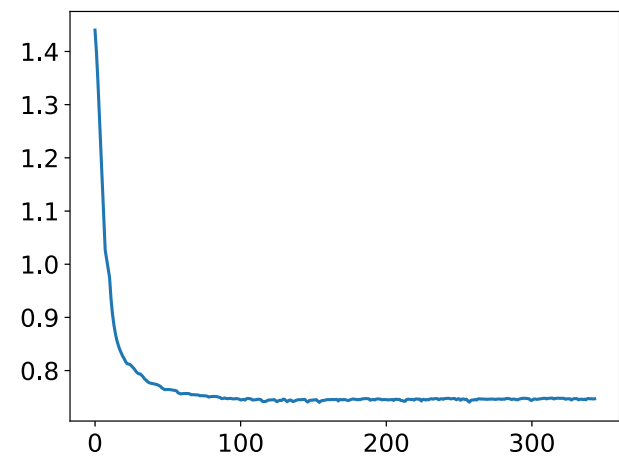

(A)

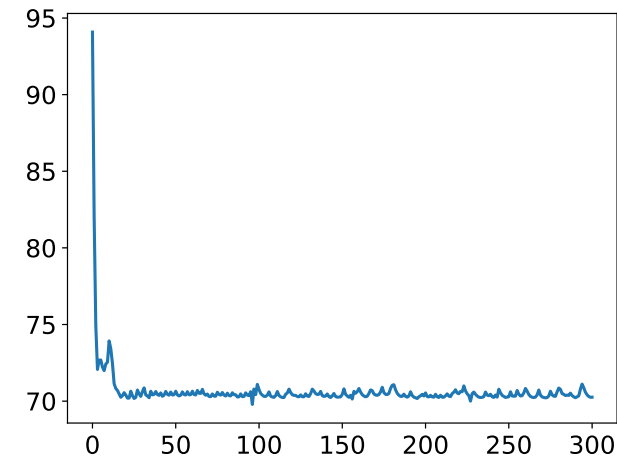

(B)

Figure 26. Optimization history for the 2-d cantilever optimization problem (4.14) of Section 4.3.1 without minimum thickness constraint. Final values: volume $\operatorname{Vol}(\Omega)=0.75$, and compliance $C(\Omega)=70.29$. (A) Volume $\operatorname{Vol}(\Omega)$. (B) Compliance $C(\Omega)$. 


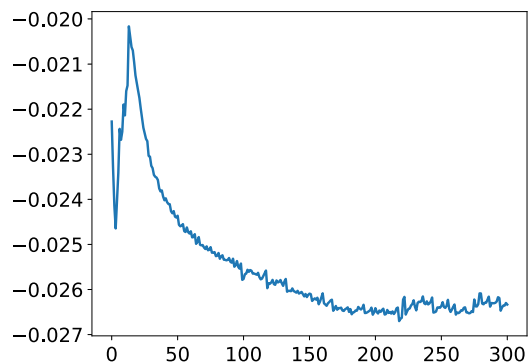

(A)

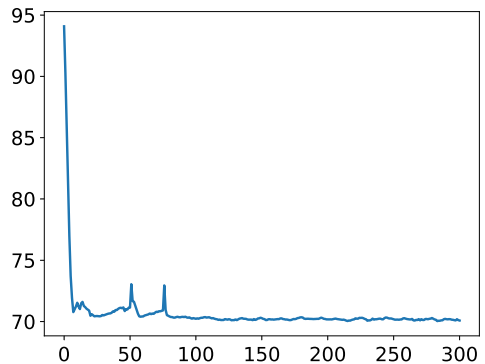

(B)

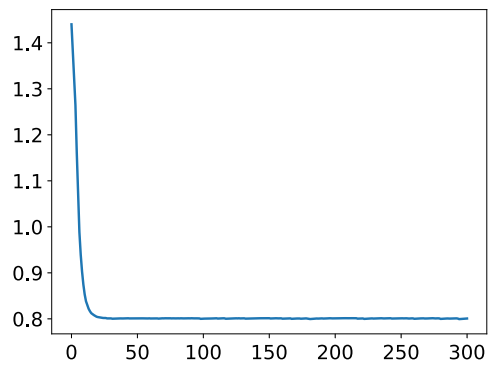

(c)

FIGURE 27. Optimization history for the 2-d cantilever optimization problem (4.14) of Section 4.3.1 with minimum thickness constraint. $\left(d_{\min }=0.1\right)$ Final values: volume $\operatorname{Vol}(\Omega)=0.80$, compliance: $C(\Omega)=70.26$. (A) Minimum thickness $P_{\mathrm{MinT}}(\Omega)$. (B) Compliance $C(\Omega)$. (c) Volume $\operatorname{Vol}(\Omega)$.

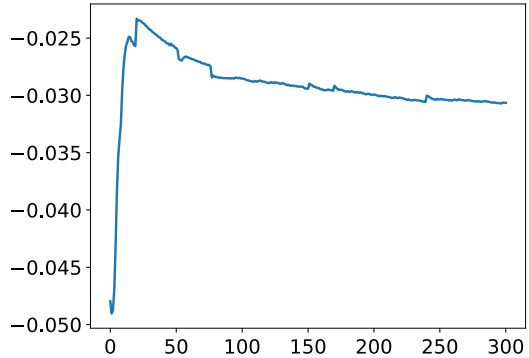

(A)

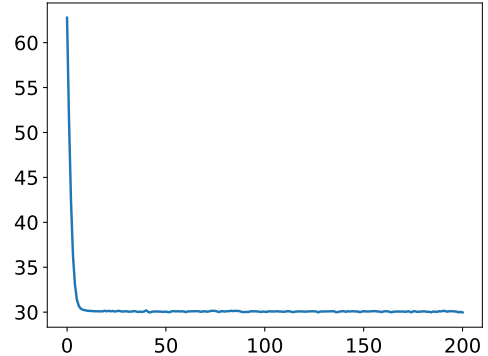

(B)

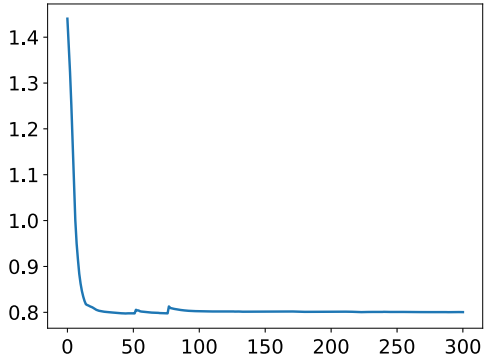

(C)

FiguRE 28. Optimization history for the 2-d cantilever optimization problem (4.14) of Section 4.3.1 with minimum thickness constraint. $\left(d_{\min }=0.2\right)$. Final values: volume $\operatorname{Vol}(\Omega)=0.80$, and compliance: $C(\Omega)=70.09$. (A) Minimum thickness $P_{\mathrm{MinT}}(\Omega)$. (в) Compliance $C(\Omega)$. (с) Volume $\operatorname{Vol}(\Omega)$.

where the term involving $d_{\Omega}^{\prime}(\theta)$ is then computed using the variational formulation (4.9).

In the forthcoming examples of Sections 4.2 .1 and 4.2.2, we solve the optimization problem of minimizing the volume $\operatorname{Vol}(\Omega)$ of the structure while imposing that the compliance $C(\Omega)$ do not exceed a given threshold $g_{\text {max }}$, as well as the maximum thickness constraint (4.11), namely:

$$
\begin{array}{ll}
\min & \operatorname{Vol}(\Omega) \\
\text { s.t. }\left\{\begin{array}{l}
C(\Omega) \leq g_{\max } \\
P_{\mathrm{MaxT}}(\Omega) \leq \frac{d_{\max }}{2} .
\end{array}\right.
\end{array}
$$

\subsubsection{Optimization of the shape of a two-dimensional arch}

Our first test-case reproduces that considered in Section 8.1.1 of [7], whose setting is displayed on Figure 17A: in a square-shaped domain $D$, the considered shapes are clamped on both their bottom left and bottom right corners, and a vertical load $\boldsymbol{g}=(0,-20)$ is applied at the middle of their bottom side.

Starting from an initial shape arbitrarily perforated with several holes, the optimization problem (4.14) is solved with and without including the maximum thickness constraint, using the numerical values $g_{\max }=7.00$ and $d_{\max }=0.12$ when they are relevant. 


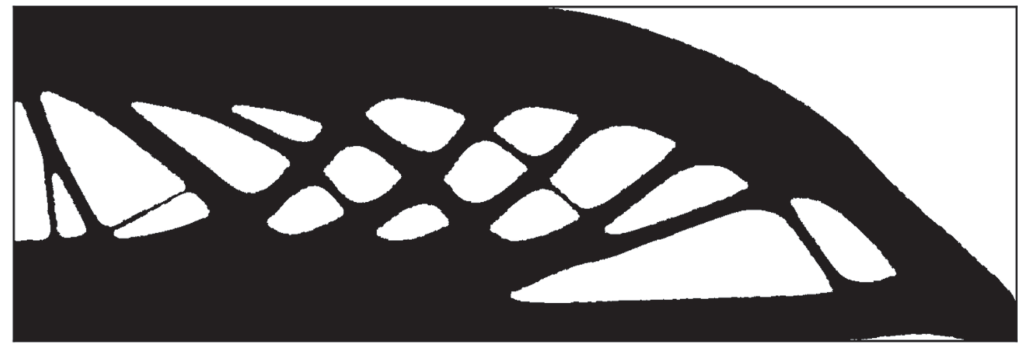

(A)

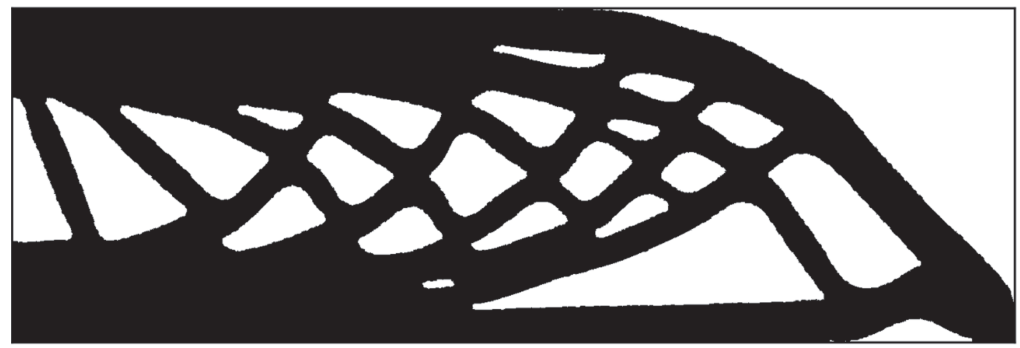

(B)

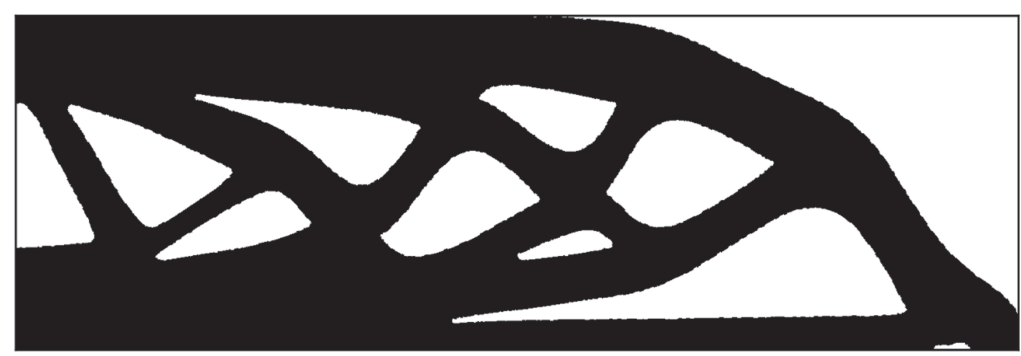

(c)

FIgURE 29. Minimum thickness optimization for a cantilever test case of Section 4.3.1. (A) Optimized shape without minimum thickness constraint. (В) Optimized shape with minimum thickness constraint $\left(d_{\min }=0.1\right)$. (c) Optimized shape with minimum thickness constraint $\left(d_{\min }=0.2\right)$.

The resulting optimized shapes in both cases are displayed on Figures 17B and 17C. Several intermediate shapes as well as the convergence histories of the computation are displayed on Figures 18-20. The obtained shapes are quite analogous to those obtained by $[7,50]$ where the calculation of the shape derivative of $P_{\operatorname{MaxT}}(\Omega)$ relied on a direct numerical integration along rays.

\subsubsection{Optimization of a two-dimensional $M B B$-Beam}

We consider now the classical MBB-Beam test-case depicted on Figure 21A: in a box $D$ with dimensions $3 \times 1$, a material shape $\Omega$ is constrained to no horizontal motion on the left boundary, and to no vertical motion on the bottom right corner. A vertical load $\boldsymbol{g}=(0,-10)$ is applied on the top left corner, and the optimization problem (4.14) is considered again, with the numerical values for the thresholds $g_{\max }=30.00$ and $d_{\max }=0.16$.

The resulting optimized shapes with and without including the maximum thickness constraint in (4.14) are represented on Figures 21B and 21C and the convergence histories of the computation are shown on Figures 22 and 23. 


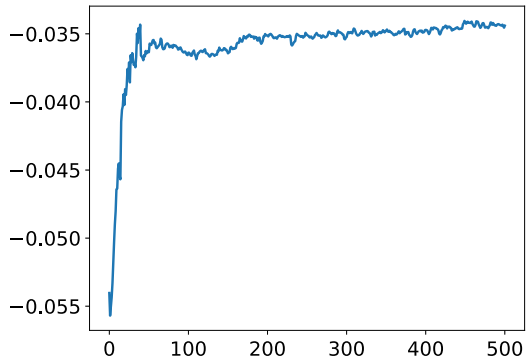

(A)

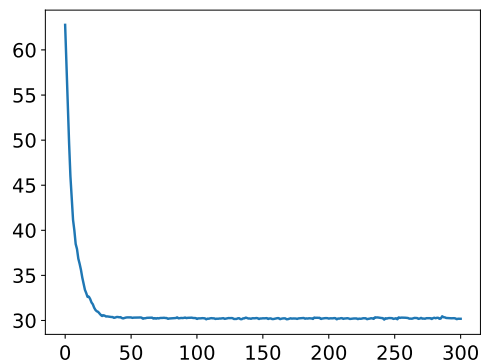

(B)

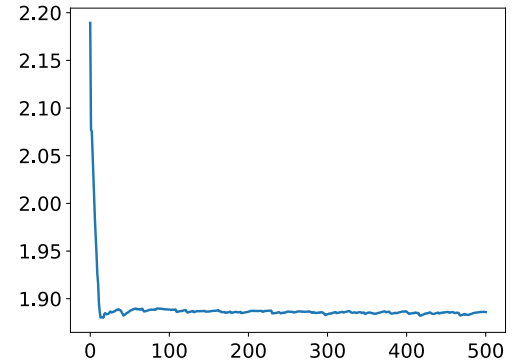

(C)

Figure 30. Convergence histories for the MBB-Beam test-case of Section 4.3.1 with minimum thickness constraint. $\left(d_{\min }=0.1\right)$. Final values: volume $\operatorname{Vol}(\Omega)=1.89$, and compliance $C(\Omega)=$ 30.17. (A) Minimum thickness $P_{\mathrm{MinT}}(\Omega)$. (B) Compliance $C(\Omega)$. (C) Volume $\operatorname{Vol}(\Omega)$.

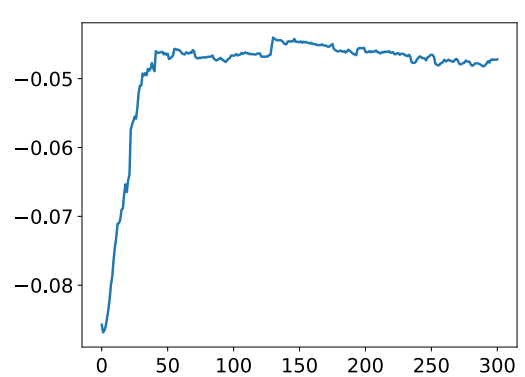

(A)

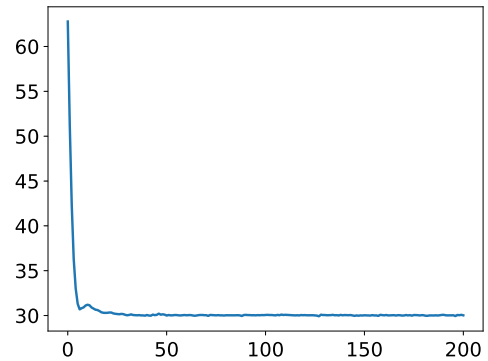

(B)

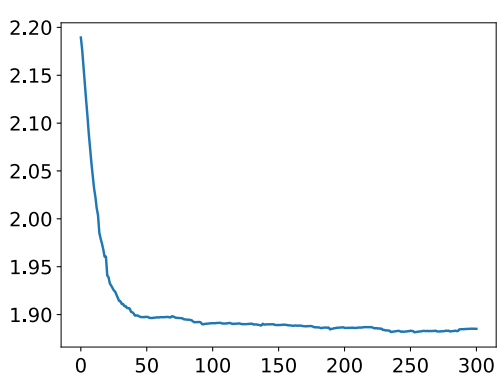

(C)

Figure 31. Convergence curves for the MBB-Beam test-case of Section 4.3.1 with minimum thickness constraint $\left(d_{\min }=0.2\right)$. Final values: volume $\operatorname{Vol}(\Omega)=1.89$, and compliance $C(\Omega)=$ 30.18. (A) Minimum thickness $P_{\mathrm{MinT}}(\Omega)$. (B) Compliance $C(\Omega)$. (C) Volume $\operatorname{Vol}(\Omega)$.

\subsection{Shape optimization examples under a minimum thickness constraint}

We now turn to the implementation of a minimum thickness constraint thanks to our variational method. Following $[7,50]$, we say that a shape $\Omega$ has minimum thickness greater than $d_{\min }$ if

$$
\forall y \in \partial \Omega, \zeta_{-}(y)<-d_{\min } / 2 .
$$

In other words, the boundary $\partial \Omega$ is at a minimum distance $d_{\min } / 2$ of the part $\Sigma \cap \Omega$ of the skeleton located inside the shape.

Enforcing a minimum thickness as a hard constraint (i.e. rather than a penalty term in the objective function) is by no means a straightforward task, because:

(1) Our definition (4.15) of minimum thickness involve the distance to the skeleton $\zeta_{-}$, which is not differentiable with respect to the shape,

(2) It is not clear how to formulate (4.15) by mean of a penalty functional such as (4.11) to penalize localizations on the shape that do not meet the thickness requirement,

(3) Even if we were able to enforce the constraint at each iteration, such would prevent topology changes to occur naturally, which would be an issue in numerical practice.

We propose in the following a more flexible setting to enforce such a requirement in a structural optimization problem. Elaborating on ideas proposed in $[16,17,47,50]$, the minimum thickness requirement is implemented 

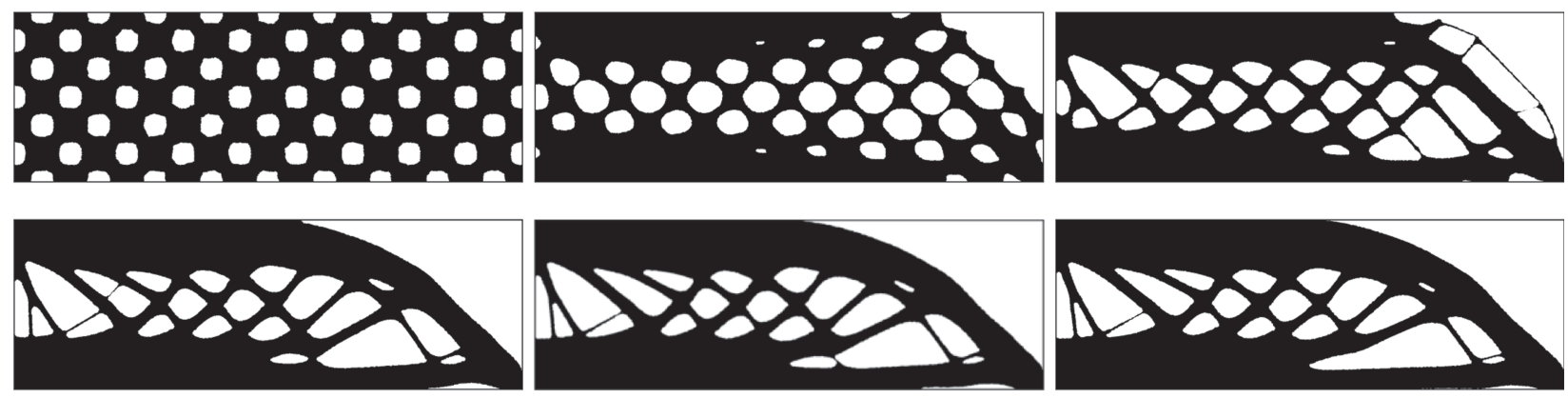

(A)
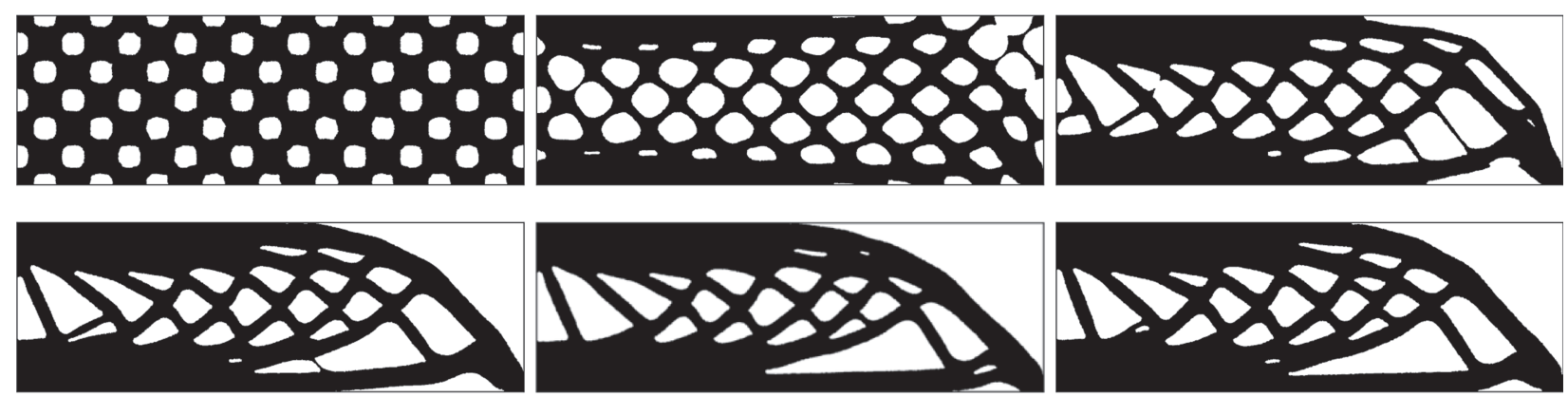

(B)
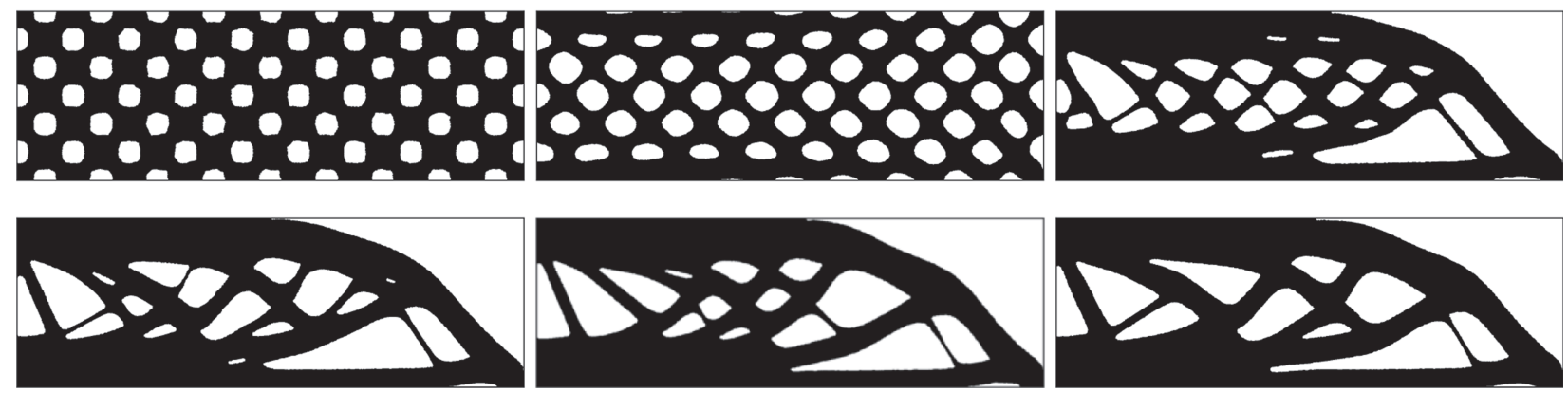

(c)

Figure 32. Optimization histories for the MBB beam test-case with minimum thickness constraints of Section 4.3.2: Iterations 0, 10, 40, 100, 150 and 200. (A) No minimum thickness constraint (setting of Fig. 21B). (в) Minimum thickness constraint with $d_{\min }=0.1$. (с) Minimum thickness constraint with $d_{\min }=0.2$.

in the objective function rather than in the constraints: we minimize a penalty functional $P_{\mathrm{MinT}}(\Omega)$ for the minimum thickness under constraints on the volume and compliance of shapes, i.e. we solve:

$$
\begin{array}{ll}
\min & P_{\mathrm{MinT}}(\Omega) \\
\text { s.t. }\left\{\begin{array}{l}
C(\Omega) \leq g_{\max } \\
\operatorname{Vol}(\Omega) \leq V_{\max }
\end{array}\right.
\end{array}
$$

This strategy is expected to work because our optimization algorithm is designed to satisfy violated constraints first, before then attempting to reduce the objective function while maintaining the constraints respected. 
The penalty functional $P_{\mathrm{MinT}}(\Omega)$ we considered in our case when solving (4.16) is taken from [50] and is specially designed to have a zero derivative when the constraint (4.15) is satisfied:

$$
P_{\mathrm{MinT}}(\Omega)=-\int_{\Omega} d_{\Omega}^{2} \max \left(d_{\Omega}+d_{\min } / 2,0\right)^{2} \mathrm{~d} x .
$$

The shape derivative of $P_{\mathrm{MinT}}(\Omega)$ is given by

$$
P_{\text {MinT }}^{\prime}(\Omega)(\theta)=-\int_{\Omega} 2\left(d_{\Omega} \max \left(d_{\Omega}+d_{\min } / 2,0\right)^{2}+d_{\Omega}^{2} \max \left(d_{\Omega}+d_{\min } / 2,0\right)\right) d_{\Omega}^{\prime}(\theta) \mathrm{d} x .
$$

Note that an increase in perimeter entails a decrease in the value of $P_{\mathrm{MinT}}(\Omega)$, but this behavior is tempered in our case by the volume constraint. Variants can be considered to address such an issue, and we refer to [50] for the details.

\subsubsection{Optimization of the shape of a 2-d cantilever beam}

We first consider the classical two-dimensional cantilever benchmark example, as depicted on Figure 24: in a box $D$ with size $2 \times 1$, shapes $\Omega$ are clamped on their left-hand side, and a vertical load $\boldsymbol{g}=(0,-10)$ is applied on the middle of their right-hand side.

The optimization problem (4.16) is solved with the parameter values $g_{\max }=70.00$ and $V_{\max }=0.80$. The resulting optimized shapes are displayed on Figure 25 without including minimum thickness constraint (we minimize the volume $\operatorname{Vol}(\Omega)$ subject to the compliance constraint as in (4.14)), and with the minimum thickness for two different values of $d_{\min }$. The corresponding convergence histories are shown on Figures 26-28.

\subsubsection{Shape optimization of a 2-d MBB Beam under a minimum thickness constraint}

We now apply the same methodology on the MBB beam test-case of Section 4.2.2. Optimized shapes are compared on Figure 29 without minimum thickness constraint (the result being that of Fig. 21B), and for two values of $d_{\min }$. The corresponding convergence histories are shown on Figures 22-31. Finally, some intermediate shapes of the optimization process are reprinted on Figure 32.

One observes that for the last case of Figure $30 \mathrm{C}$ with $d_{\min }=0.2$, the minimum thickness constraint is not satisfied everywhere but a substantial improvement is visible over the first design of Figure 30c. Notably, this approach is sufficiently flexible to guide the optimization path towards shapes with very different topologies.

Acknowledgements. This work was supported by the Association Nationale de la Recherche et de la Technologie (ANRT) [grant number CIFRE 2017/0024]. G. A. is a member of the DEFI project at INRIA Saclay Ile-de-France. The work of C.D. is partially supported by the IRS-CAOS grant from Université Grenoble-Alpes. The authors thank the reviewers for their helpful comments in the revision of this manuscript.

\section{REFERENCES}

[1] G. Allaire, In: Vol. of 58 Conception optimale de structures. Springer (2007).

[2] G. Allaire, F. Jouve and A.-M. Toader, Structural optimization using sensitivity analysis and a level-set method. J. Comput. Phys. 194 (2004) 363-393.

[3] G. Allaire, F. De Gournay, F. Jouve, and A.-M. Toader. Structural optimization using topological and shape sensitivity via a level set method. Control Cybern. 34 (2005) 59.

[4] G. Allaire, C. Dapogny and P. Frey, Shape optimization with a level set based mesh evolution method. Comput. Methods Appl. Mech. Eng. 282 (2014) 22-53.

[5] G. Allaire, C. Dapogny, G. Delgado and G. Michailidis, Multi-phase structural optimization via a level set method. ESAIM: COCV 20 (2014) 576-611.

[6] G. Allaire, F. Jouve and G. Michailidis, Molding direction constraints in structural optimization via a level-set method. In: Variational Analysis and Aerospace Engineering. Springer (2016) 1-39.

[7] G. Allaire, F. Jouve and G. Michailidis, Thickness control in structural optimization via a level set method. Struct. Multi. Optim. 53 (2016) 1349-1382. 
[8] L. Ambrosio, Geometric evolution problems, distance function and viscosity solutions. In: Calculus of Variations and Partial Differential Equations. Springer (2000) 5-93.

[9] N. Amenta, S. Choi and R.K. Kolluri, The power crust, unions of balls, and the medial axis transform. Comput. Geom. 19 (2001) 127-153.

[10] D. Attali, J.-D. Boissonnat and H. Edelsbrunner, Stability and computation of medial axes-a state-of-the-art report. In: Mathematical Foundations of Scientific Visualization, Computer Graphics, and Massive Data Exploration. Springer (2009) 109-125.

[11] P. Azérad, Equations de Navier-Stokes en bassin peu profond. Ph.D. thesis, Université de Neuchâtel (1995).

[12] P. Azérad and J. Pousin, Inégalité de poincaré courbe pour le traitement variationnel de l'équation de transport. C.R. Acad. Sci. Ser. 1: Math. 322 (1996) 721-727.

[13] A. Bensalah, Une approche nouvelle de la modélisation mathématique et numérique en aéroacoustique par les équations de Goldstein et applications en aéronautique. Ph.D. thesis, Université Paris Saclay (2018).

[14] G. Bellettini, Lecture Notes on Mean Curvature Flow: Barriers and Singular Perturbations. In Vol. 12. Springer (2014).

[15] P. Cannarsa and P. Cardaliaguet, Representation of equilibrium solutions to the table problem of growing sandpiles. J. Eur. Math. Soc. 6 (2004) 435-464.

[16] S. Chen, M.Y. Wang and A.Q. Liu, Shape feature control in structural topology optimization. Comput.-Aided Des. 40 (2008) 951-962.

[17] G. Cheng, Y. Mei and X. Wang, A feature-based structural topology optimization method. In: IUTAM Symposium on Topological Design Optimization of Structures, Machines and Materials. Springer (2006) 505-514.

[18] C. Chicone, Ordinary Differential Equations with Applications. Springer New York (1999).

[19] D.L. Chopp, Another look at velocity extensions in the level set method. SIAM J. Sci. Comput. 31 (2009) $3255-3273$.

[20] C. Dapogny and P. Frey, Computation of the signed distance function to a discrete contour on adapted triangulation. Calcolo 49 (2012) 193-219.

[21] C. Dapogny, C. Dobrzynski and P. Frey, Three-dimensional adaptive domain remeshing, implicit domain meshing, and applications to free and moving boundary problems. J. Comput. Phys. 262 (2014) 358-378.

[22] C. Dapogny, R. Estevez, A. Faure and G. Michailidis, Shape and topology optimization considering anisotropic features induced by additive manufacturing processes. Hal Preprint: https://hal.archives-ouvertes.fr/hal-01660850/ (2017).

[23] C. Dapogny, A. Faure, G. Michailidis, G. Allaire, A. Couvelas and R. Estevez, Geometric constraints for shape and topology optimization in architectural design. Comput. Mech. 59 (2017) 933-965.

[24] G. David and S. Semmes, Uniform rectifiability and singular sets. Ann. Inst. Henri Poincaré (C) Non Linear Anal. 13 (1996) 383-443.

[25] M.C. Delfour and J.P. Zolésio, Shape analysis via distance functions. J. Funct. Anal. 123 (1994) 129-201.

[26] M.C. Delfour and J.-P. Zolesio, Shapes and Geometries: Metrics, Analysis, Differential Calculus, and Optimization. In Vol. 22. SIAM (2011).

[27] T.K. Dey and W. Zhao, Approximate medial axis as a voronoi subcomplex. In: Proceedings of the Seventh ACM Symposium on Solid Modeling and Applications. ACM (2002) 356-366.

[28] R.J. DiPerna and P.-L. Lions, Ordinary differential equations, transport theory and sobolev spaces. Invent. Math. 98 (1989) $511-547$.

[29] D.A. Di Pietro and A. Ern, Mathematical Aspects of Discontinuous Galerkin Methods. In Vol. 69. Springer Science \& Business Media (2011).

[30] J. Duoandikoetxea, Forty years of muckenhoupt weights. In: Function Spaces and Inequalities. Matfyzpress, Praga (2013) 23-75.

[31] B. Erem and D.H. Brooks, Differential geometric approximation of the gradient and hessian on a triangulated manifold. In: Vol. 504 of Proceedings/IEEE International Symposium on Biomedical Imaging: From Nano to Macro. NIH Public Access (2011).

[32] A. Ern and J.-L. Guermond, Theory and Practice of Finite Elements. In Vol. 159. Springer Science \& Business Media (2013).

[33] W.D. Evans, Weighted sobolev spaces. Bull. London Math. Soc. 18 (1986) 220-221.

[34] L.C. Evans and R.F. Gariepy, Measure theory and fine properties of functions. In: Studies in Advanced Mathematics. CRC Press (1992).

[35] F. Feppon and P.F.J. Lermusiaux, A geometric approach to dynamical model order reduction. SIAM J. Matrix Anal. App. 39 (2018) 510-538.

[36] F. Feppon, G. Allaire, F. Bordeu, J. Cortial and C. Dapogny, Shape optimization of a coupled thermal fluid-structure problem in a level set mesh evolution framework. Hal Preprint: https://hal.archives-ouvertes.fr/hal-01686770/ (2018).

[37] F. Feppon, G. Allaire and C. Dapogny, Null space gradient flows for constrained optimization with applications to shape optimization. Hal Preprint: https://hal archives-ouvertes.fr/hal-01972915/ (2019).

[38] J.K. Guest, Imposing maximum length scale in topology optimization. Struct. Multi. Optim. 37 (2009) $463-473$.

[39] F. Hecht, New development in freefem++. J. Numer. Math. 20 (2013) 251-265.

[40] A. Henrot and M. Pierre, Variation et optimisation de formes: une analyse géométrique. In Vol. 48. Springer Science \& Business Media (2006).

[41] M. Jensen, Discontinuous Galerkin methods for Friedrichs systems with irregular solutions. Ph.D. thesis, University of Oxford (2005). 
[42] T. Krainer and B.W. Schulze, Weighted Sobolev Spaces. Springer (1985).

[43] A. Kufner and B. Opic, How to define reasonably weighted sobolev spaces. Commentationes Math. Univ. Carolinae 25 (1984) $537-554$

[44] S. Lang, Fundamentals of Differential Geometry. In Vol. 191. Springer Science \& Business Media (2012).

[45] Y.Y. Li and L. Nirenberg, The distance function to the boundary, finsler geometry, and the singular set of viscosity solutions of some hamilton-jacobi equations. Commun. Pure Appl. Math. 58 (2005) 85-146.

[46] J. Liu and Y. Ma, A survey of manufacturing oriented topology optimization methods. Adv. Eng. Softw. 100 (2016) $161-175$.

[47] J. Luo, Z. Luo, S. Chen, L. Tong and M.Y. Wang, A new level set method for systematic design of hinge-free compliant mechanisms. Comput. Methods Appl. Mech. Eng. 198 (2008) 318-331.

[48] C. Mantegazza and A.C. Mennucci, Hamilton-Jacobi equations and distance functions on riemannian manifolds. Appl. Math. Optim. 47 (2003) 1-25.

[49] M. Meyer, M. Desbrun, P. Schröder and A.H. Barr, Discrete differential-geometry operators for triangulated 2-manifolds. In: Visualization and Mathematics III. Springer (2003) 35-57.

[50] G. Michailidis, Manufacturing constraints and multi-phase shape and topology optimization via a level-set method. Ph.D. thesis, Ecole Polytechnique (2014).

[51] F. Murat and J. Simon, Etude de problèmes d'optimal design. In: IFIP Technical Conference on Optimization Techniques. Springer (1975) 54-62.

[52] E. Peynaud, Rayonnement sonore dans un écoulement subsonique complexe en régime harmonique: analyse et simulation numérique du couplage entre les phénomènes acoustiques et hydrodynamiques. Ph.D. thesis, Toulouse, INSA (2013).

[53] J.C. Robinson, Infinite-dimensional Dynamical Systems: An Introduction to Dissipative Parabolic PDEs and the Theory of Global Attractors. In Vol. 28. Cambridge University Press, Cambridge (2001).

[54] W. Rudin, Real and Complex Analysis. Tata McGraw-Hill Education, New York, NY (2006).

[55] S. Rusinkiewicz, Estimating curvatures and their derivatives on triangle meshes. In: Proceedings. 2nd International Symposium on 3D Data Processing, Visualization and Transmission, 2004. 3DPVT 2004. IEEE (2004) 486-493.

[56] J. Schropp and I. Singer, A dynamical systems approach to constrained minimization. Numer. Funct. Anal. Optim. 21 (2000) $537-551$.

[57] J.A. Sethian, A fast marching level set method for monotonically advancing fronts. Proc. Nat. Acad. Sci. 93 (1996) $1591-1595$.

[58] J. Sokolowski and J.-P. Zolésio, Introduction to shape optimization. In: Vol. 16 of Springer Series in Computational Mathematics. Springer-Verlag, Berlin (1992).

[59] L. Tartar, An Introduction to Sobolev Spaces and Interpolation Spaces. In Vol. 3. Springer Science \& Business Media (2007).

[60] B.O. Turesson, Nonlinear Potential Theory and Weighted Sobolev Spaces. Springer (2007).

[61] M.Y. Wang, X. Wang and D. Guo, A level set method for structural topology optimization. Comput. Methods Appl. Mech. Eng. 192 (2003) 227-246.

[62] G.H. Yoon, S. Heo and Y.Y. Kim, Minimum thickness control at various levels for topology optimization using the wavelet method. Int. J. Solids Struct. 42 (2005) 5945-5970.

[63] H. Zhao, A fast sweeping method for eikonal equations. Math. Comput. 74 (2005) 603-627. 